\title{
Children's Acquisition of the Mappings among the Number Representations
}

\author{
By \\ Carolina Jiménez Lira
}

\begin{abstract}
A thesis submitted to the Faculty of Graduate and Postdoctoral Affairs in partial fulfillment of requirements

for the degree of
\end{abstract}

\author{
Doctor of Philosophy \\ in
}

Developmental Psychology

Carleton University

Ottawa, Ontario, Canada

Carolina Jiménez Lira ㄷ 2016 


\begin{abstract}
To understand mathematics, children need to learn how the verbal and written number symbols are related to each other and how they are used to represent quantity. How are these mapping skills acquired? Across three studies I examined the relationship between children's mapping skills and their quantitative and symbolic precursor skills. I also assessed whether children's mapping skills were related to more advanced numerical skills such as symbolic number comparison (Study 1,2), and whether the mapping skills improved with training (Study 3).
\end{abstract}

The Pathways Model of early numeracy development (LeFevre, Fast et al., 2010) was extended to include children's mapping skills as mediators between early quantitative and symbolic number knowledge and symbolic number comparison. The model was assessed in two samples of Canadian children (Study 1, $n=62$; Study 2, $n=$ 45) and a sample of Mexican children (Study 2, $n=57$ ). Results from both studies show that verbal counting, identification of symbolic number, and understanding of cardinality were important predictors of children's mapping abilities. Children's ability to associate Arabic digits to the corresponding quantity representation was predictive of their symbolic number comparison skills. Children first learned to associate number words to digits, then number words to quantities, and finally digits to quantities.

Study 3 was an intervention study in which the goal was to assess whether enhancing children's cardinality skills would result in an improvement to their symbolquantity mappings. No significant effects of the training procedure were found. Children in this study had relatively strong early numeracy skills and thus the lack of learning may have reflected the lack of variability in their existing knowledge. 
Finally, Canadian and Mexican preschool children's early numeracy skills were assessed in relation to the home learning environment. Significant relationships between reported frequency of home numeracy practices and numeracy skills were found for the Canadian but not for the Mexican children, whereas maternal education was found to be a significant predictor of numeracy skills in both countries.

In conclusion, the three studies included in the present research provide insight into how mathematical skills unfold from the earliest symbolic and quantitative skills. 


\section{ACKNOWLEDGEMENTS}

To God for giving me the opportunity to pursue this dream and the strength to complete it, for guiding me along the way, and for placing the most wonderful people in my path. To my daughter Victoria for her patience and love. To my family in Mexico who, even through tough times always encouraged me to continue. To the very special professor who has been a great example for me, whose research has inspired me the most, who encouraged me to do more, who always had my back and believed in me at times when I did not: my supervisor Dr. Jo-Anne LeFevre. I will forever be grateful for the opportunity to work with and learn from such a wonderful teacher and human being. For your time, kindness, patience, guidance, wise advice, unending support, and friendship I can only say THANK YOU!! To my lab mates for their wonderful friendship and support throughout this journey. To Dr. Andrea Howard and Dr. Deepthi Kamawar for their insightful suggestions and support. To Dr. Rigoberto Marín Uribe whose help enabled me to carry out the research in Mexico. To Brianna Herdman, Heather Douglas, Miranda Carver, and Kelsey MacKay for their help with the data collection. To Etelle Bourassa who was always willing to provide assistance with a smile on her face.

Finally, I thank the Mexican National Council of Science and Technology (CONACyT) and the Department of Psychology at Carleton University for the funding that enabled me to reach this goal. 


\section{TABLE OF CONTENTS}

List of Tables ............................................................................................................................ vii

List of Figures ............................................................................................................................... $x$

CHAPTER 1: INTRODUCTION......................................................................................... 1

CHAPTER 2: EARLY NUMERACY DEVELOPMENT .......................................... 6

Introduction ..................................................................................................................... 6

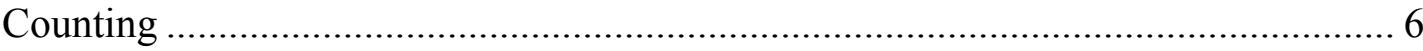

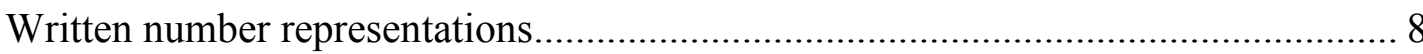

Models of early numeracy development ………………..................................... 9

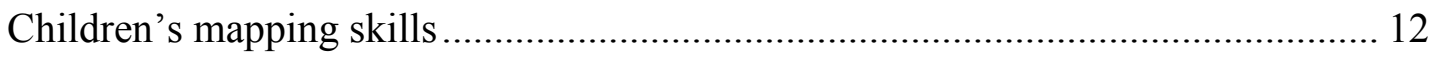

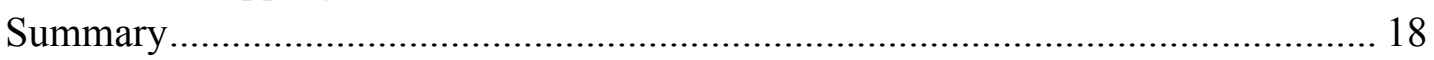

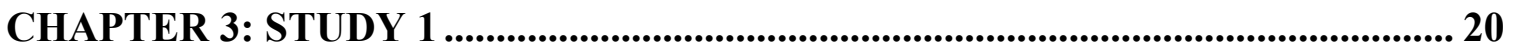

Introduction ............................................................................................................................. 22

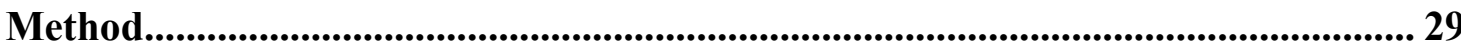

Results .......................................................................................................................... 44

Discussion .............................................................................................................................. 61

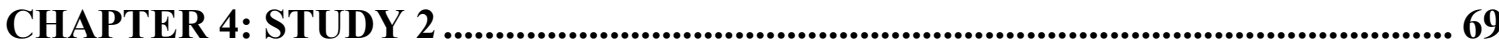

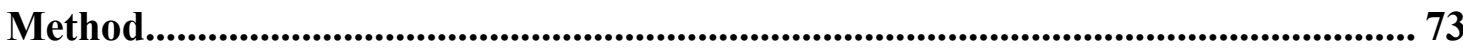

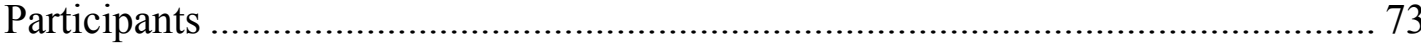

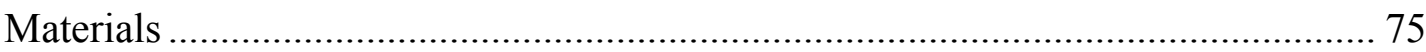

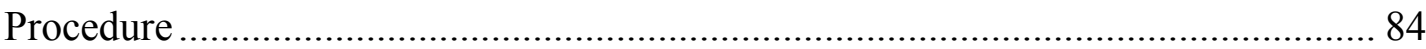

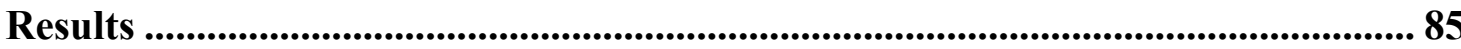

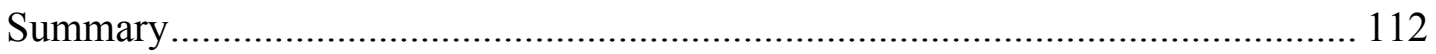

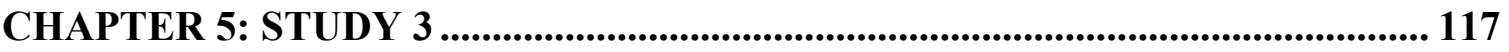

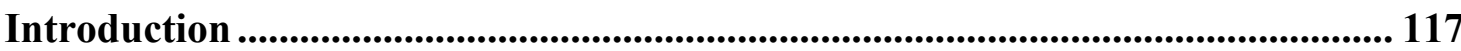

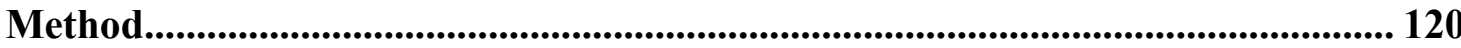

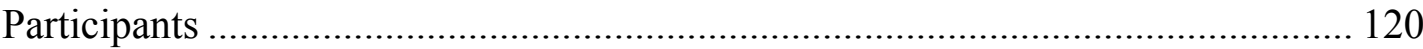

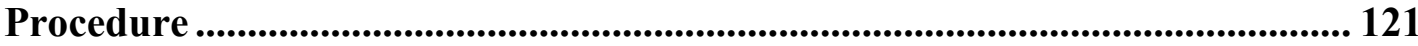

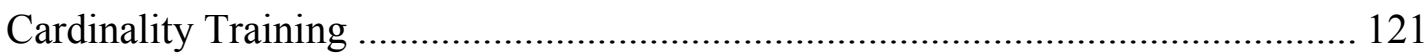

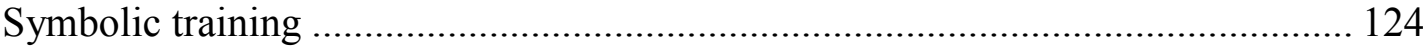

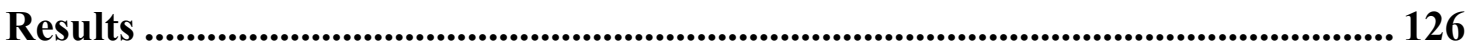

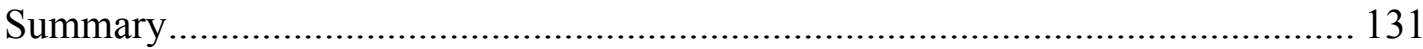

CHAPTER 6: STUDY 2, HOME AND SCHOOL FACTORS ............................. 135

Introduction .......................................................................................................................... 135

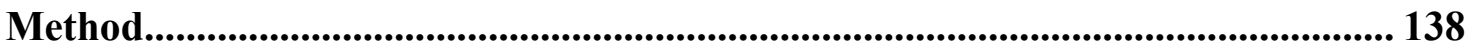

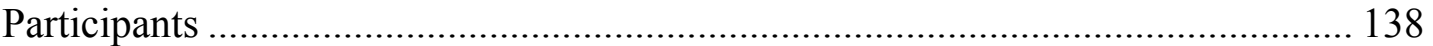

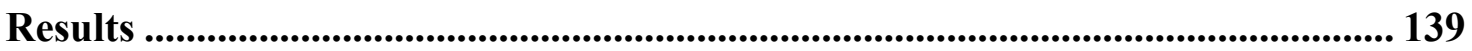

Do academic expectations differ between Mexican and Canadian parents? ........... 140 
Are maternal education and parental academic expectations predictive of the reported frequency of engaging in home numeracy activities in both countries? ... 148

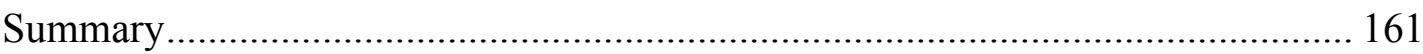

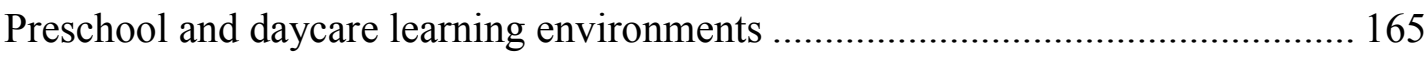

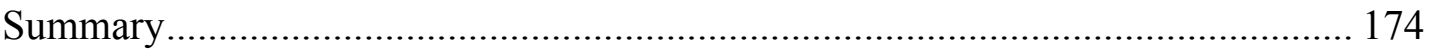

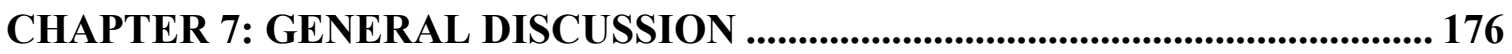

Limitations .......................................................................................................................... 188

Conclusions ......................................................................................................................... 190

REFERENCES.................................................................................................................. 193

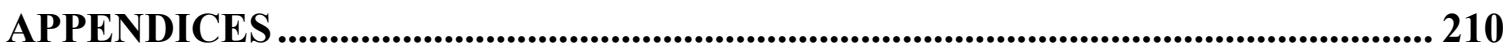




\section{List of Tables}

Table 3.1. Performance on all tasks. 33

Table 3.2. Stimuli for the tasks requiring children to select between representations 36

Table 3.3. Inferential statistics for analyses of the pairs of mapping tasks. 50

Table 3.4. Zero order correlations among age, verbal counting, all composite variables, and comparison tasks. 53

Table 3.5. Path analysis: Standardized direct, indirect and total effects. 54

Table 3.6. Path analysis: Other significant (standardized) indirect effects. 57

Table 3.7. Percentage of children who performed above chance on each of the three sets of mapping tasks. 60

Table 4.1. Highest level of education for Mexican and Canadian parents. 74

Table 4.2. Distribution of teachers who completed the questionnaire and the number of children from each preschool or daycare. 75

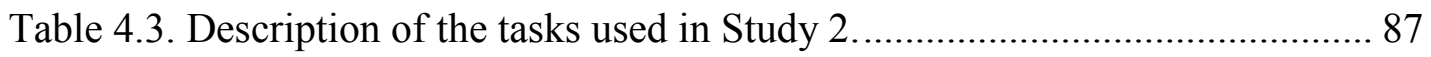

Table 4.4. Mapping task means by set size and country.................................... 90

Table 4.5. Statistics for the Task by Size by Country ANOVA.............................. 91

Table 4.6. Performance on both versions of the KeyMath by country................... 96

Table 4.7. Performance on verbal comparison by distance and country (maximum score is 10$)$.

Table 4.8. Zero order correlations among tasks and home numeracy practices (mapping combined)

Table 4.9. Partial correlations (age controlled) among numeracy tasks. 99

Table 4.10. Path analysis: Unstandardized direct, total indirect and total effects for the multi-group path analyses by country. 105

Table 4.11. Path analysis: Other significant (unstandardized) indirect effects for the Canadian sample. 106 
Table 4.12. Path analysis: Other significant (unstandardized) indirect effects for the Mexican sample. 106

Table 4.13. Percentage of children who performed above chance on each of the three sets of mapping tasks in both countries. 110

Table 4.14. Contingency table for word-digit, digit-quantity mapping tasks in both countries.

Table 4.14. Contingency table for word-quantity, digit-quantity mapping tasks in both countries.

Table 5.1. Descriptive statistics for all measures on pre-test and post-test in Study 3.

Table 5.2. Statistics for Analyses of Covariance of numeracy performance controlling for pre-test scores.

Table 6.1. Academic expectations by country.

Table 6.2. Home numeracy practices by country.

Table 6.3. Literacy home practice by country.

Table 6.4. Inferential statistics for analyses of home learning practices.

Table 6.5. Zero order correlations among education, academic expectations, and home practices.

Table 6.6. Results from regression analyses predicting home numeracy practices in both countries (unstandardized coefficients).

Table 6.7. Results from regression analyses predicting home literacy practices in both countries (unstandardized coefficients).

Table 6.8. Mexican sample zero order correlations among tasks and home numeracy practices (mapping combined orders).

Table 6.9. Canadian sample zero correlations among tasks and home numeracy practices (mapping combined).

Table 6.10. Results from regression analyses predicting counting outcomes for the Canadian and Mexican sample. 158

Table 6.11 Results from regression analyses predicting mapping outcomes. 159 
Table 6.12. Results from regression analyses predicting more complex math outcomes for the Canadian and Mexican sample.

Table 6.14. Averaged Teachers' Reports of Classroom numeracy practice by country.

Table 6.15. Averaged teachers' reports of literacy practice by country. 173 


\section{List of Figures}

Figure 3.1. Mappings among the three representations of number........................ 23

Figure 3.2. Hypothesized relations among precursor, mapping and comparison skills.

Figure 3.3. Examples of how the mapping tasks appeared to the children.............. 32

Figure 3.4. Number correct for each pair of mapping tasks.

Figure 3.5. Path model of the relations among children's precursor skills, mapping performance, and comparison tasks. 56

Figure 4.1. Examples of the mapping task stimuli 78

Figure 4.2. Percentage of children who counted successfully to each number (i.e., highest correct count).

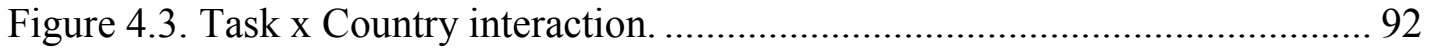

Figure 4.4. Performance by set size and country. ............................................. 92

Figure 4.5. Performance on the three tasks by set size for both countries............... 94

Figure 4.6 Hypothesized model of relations among numeracy tasks based on the results from Study 1 . 101

Figure 4.7. Significant standardized effects from the multi-group path analyses. . 104

Figure 5.1 Predicted patterns of improvement by training conditions. 119

Figure 5.2. Examples the cards used for the cardinality training. 122

Figure 5.3. Examples of the cards used for the symbolic training condition 125

Figure 5.4. Results for pre- and post-test performance on digit-word mapping by training condition.

Figure 5.5. Results for pre- and post-test performance on digit-quantity mapping by training condition.

Figure 6.1. Means for parent-reported frequencies of engaging in numeracy and literacy activities by country.

Figure 6.2. Caterpillar displaying the written Arabic digits and the corresponding quantities. 
Figure 6.3. Distribution of responses for items on which Canadian teachers reported greater frequencies than Mexican teachers.

Figure 6.4. Distribution of responses for items on which Mexican teachers reported

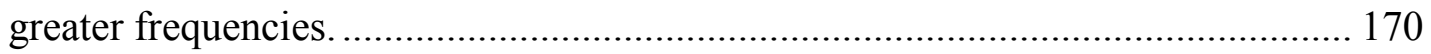

Figure 6.5. Distribution of teacher-reported frequencies of asking children to print

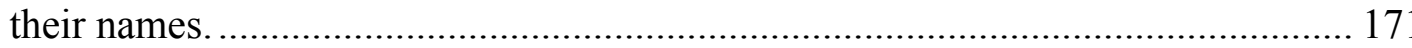




\section{CHAPTER 1: INTRODUCTION}

Mathematics, expressed in visual symbols, is the only language that is used all over the world. Even when numbers are expressed in English, Spanish, or German, they always refer to the same underlying constructs. Accordingly, children from literate cultures must acquire knowledge of how the verbal and written number symbols are used to represent quantities in order to develop the more advanced skills of this universal language (e.g., arithmetic, fractions, algebra). Specifically, before children can understand and solve more elaborate math problems, they must understand how each verbal number word is associated with a specific digit, and how the verbal number words and written digits are associated with a unique quantity. The present research examines these associations or mappings among verbal number words, written digits, and nonsymbolic exact quantities in two-to-four-year-old children.

To explore whether the mapping skills follow a similar developmental pattern in children with different cultural and educational backgrounds, samples of Canadian and Mexican children participated. Specifically, I examined the associations among number representations and their relations to other numeracy skills for both groups of children. My research provides information that could be useful for the development of assessment tools that could allow teachers to identify children at risk for developing math difficulties during the preschool years. Furthermore, little is known about the development of Mexican children's early mapping skills or about Mexican parents' approach to early numeracy education.

The development of the mappings among the number representations is presumably the result of a lengthy process during which children must integrate their 
knowledge of non-symbolic quantities and of the verbal and written symbolic number representations. This process begins very early in life. Researchers agree that children can discriminate both exact and approximate quantities from birth (Feigenson, Dehaene, \& Spelke, 2004; Piazza, 2010). Furthermore, by the age of two, most children have acquired some knowledge of the symbolic number representations; typically, by this age, children are able to repeat the counting sequence up to 10 (Fuson, 1988), and identify some Arabic digits (Mix, 2009). At this point, children begin to slowly associate the first few number words with the exact quantities they represent (i.e., they begin to understand the cardinality principle; Wynn, 1992). In the present research, children's verbal counting, their ability to identify verbal and written representations of number and their knowledge of exact quantity and cardinality were considered precursor skills that allow children to create the links among the three representations of number.

To date, the sequence children follow to develop the mappings among the number representations has not been fully established. Bialystok (1992) proposed a sequence of acquisition of the mappings in which children initially learn to map digits to number words, then, number words to quantities and finally, digits to quantities. Benoit, Lehalle, Molina, Tijus, and Jouen (2013) however, found that children learn the mapping between digits and quantities prior to mapping number words to quantities and number words to digits. In contrast, Knudsen, Fischer, Henning, and Aschersleben (2015) found that the digit-quantity mapping was the last mapping to develop and that the mappings between number words and digits and number words and quantities developed simultaneously. Thus, a goal of the present research was to examine the sequence of acquisition of the mappings among the number representations. 
Knowledge of the mappings among the number representations implies that children have developed the understanding of how the verbal and written number symbols are used to represent exact quantities. Presumably then, once children have the mappings available, they are ready to use these skills in more complex math tasks. In the current research, I examined whether children's mappings between number symbols and the corresponding quantities were predictive of children's ability to perform verbal and written number comparisons. Furthermore, based on prior models of early numeracy development by LeFevre, Fast, et al. (2010) and von Aster and Shalev (2007), a model of relationships among precursor, mapping, and number comparison skills was proposed and tested. Finally, based on the results of the model, I explored whether training the precursor skills could enhance children's mapping. Knowledge of the course of development of the mapping skills, its relationship to other numeracy skills, and of whether the mapping skills can be enhanced could aid in the design of more precise and efficient interventions.

The development of children's early numeracy skills occurs within the context of the home and childcare or preschool environments. Throughout their first years of life, children are exposed to multiple learning opportunities provided by their parents and daycare or preschool teachers. Researchers have found significant relations between the development of children's early numeracy skills and their home numeracy environments for North American (Blevins-Knabe, 2008; LeFevre, Skwarchuk, Smith-Chant, Kamawar, \& Bisanz, 2009; LeFevre, Polyzoi, Skwarchuk, Fast, \& Sowinski, 2010; Skwarchuk, Sowinski, \& LeFevre, 2014; Starkey \& Klein, 2008), European (Anders et al., 2012; Kleemans, Peeters, Segers, \& Verhoeven, 2012; LeFevre, Polyzoi et al., 2010) 
and Asian children (Pan, Gauvain, Liu, \& Cheng, 2006). Similarly, math activities conducted within the daycare or preschool classrooms have been found to enhance children's numeracy skills (Choi \& Dobbs-Oates, 2014; DeHaan, Elbers, \& Leseman, 2014). To the best of my knowledge, no published research exists on the relationship between the home numeracy environments and the development of preschool children's math skills in Mexico. In the present research, Canadian and Mexican parents were asked about the frequency with which they engaged their children in numeracy and literacy activities. The goal was to replicate previous findings in the Canadian sample and to provide initial insight into Mexican children's home and learning environments and the relations to children's developing early numeracy skills. The numeracy and literacy activities that children encounter during their time in preschool or daycare may also differ between countries; especially because the approach to teaching is play-based in Canadian daycares and more formal and direct in Mexican preschools. The present research aims to provide a description of the preschool and daycare learning environments that children in both countries are exposed to.

The present thesis includes three studies and is composed of seven chapters. Chapter 1 includes the introduction in which the main issues are briefly described. Chapter 2 includes the literature review where I describe the relevant models of early numeracy development and provide an overview of prior studies that have examined children's early numeracy and mapping skills. Chapter 3 presents the results of Study 1 in which I examined the precursor skills children need to acquire before they are able to map between the number representations, the order in which the mapping skills are acquired, and the relationship between the mapping skills and the more advanced 
symbolic number comparison skills. The results of Study 1 were used as a framework to extend the work to a sample of Mexican children. The results of Study 2 are presented in two separate chapters; Chapter 4 examines Canadian and Mexican children's performance on the early numeracy assessments. Children from both countries were included to assess whether the pattern of relations between precursor, mapping and symbolic number comparison skills would differ in children with distinct cultural backgrounds and educational experiences (e.g., Mexican children had been enrolled in formal preschool for 8 months, while Canadian children had not yet attended preschool and were still in daycare). Chapter 6 examines the relationship between parent variables and children's early numeracy skills in Canada and Mexico; in addition, a description of the daycare and preschool learning environments is provided. Results from Study 1 and 2 showed that children's knowledge of exact quantity and cardinality skill was an important predictor of their digit-quantity mapping, thus, in Study 3, I explored whether children's mapping skills could be enhanced with training. Study 3 is presented in Chapter 5.

Finally, Chapter 7 presents the general conclusions and discussion. 


\section{CHAPTER 2: EARLY NUMERACY DEVELOPMENT \\ Introduction}

Children's ability to associate verbal number words and Arabic digits to their nonsymbolic quantity representations have been found to be predictive of performance on measures of more complex symbolic math skills in preschool children (Batchelor, Inglis, \& Gilmore, 2015) and on curriculum-based math assessments in elementary-school-aged children (Brankaer, Ghesquire, DeSmedt, 2014; Mundy \& Gilmore, 2009). The mappings among the number representations have been proposed to develop once children have acquired basic symbolic number skills such as verbal counting and identifying some written digits (Bialystok, 1992). However, the sequence of acquisition of these mappings has not yet been defined. Although most research suggests that children need to create the links between verbal number words and digits and between verbal number words and quantities prior to associating digits to their corresponding quantities, other researchers have found that children initially link digits to quantities (Benoit et al., 2013). It has been suggested that the contrasting pattern of results are due to differences in early educational experiences. In this chapter I present an overview of research on the development of children's symbolic number and mapping skills.

\section{Counting}

By age two, children begin to learn the ordered list of number words (Fuson, 1988). The sequence of count words however, initially has no meaning. Children gradually develop the understanding of how each word in the count sequence is related to the others and how the order of the number list allows for it to be used in determining the number of objects in a set (Carey, 2004; Sarnecka \& Carey, 2008; Wiese, 2003; Wynn, 
1992). The importance of understanding the semantic meaning of the verbal number words has been demonstrated in many studies and has been identified as a critical stage in early math development (e.g., Krajewski \& Schneider, 2009; LeFevre, Fast, et al., 2010; Purpura et al., 2013). For example, research has found that preschool children's advanced counting skills (e.g., understanding of cardinality, counting backward and forward) are longitudinally predictive of arithmetic performance (Aunola, Leskinen, Lerkkanen, \& Nurmi, 2004; Chu, van Marle, \& Geary, 2015; Manfra, Dinehart, \& Sembiante, 2014; Nguyen et al., 2016; Tobia, Bonifacci, \& Marzocchi, 2016).

The counting principles. The development of children's counting skill is determined by their ability to understand the counting principles (Gelman \& Gallistel, 1978). The one-to-one correspondence principle states that each number word should be applied to enumerate one and only one object in the set. According to the stable order principle, in order to be efficiently used for counting objects, the list of count words should be used in the pre-specified order. The cardinality principle follows from the oneto-one correspondence and stable order principle and refers to the knowledge that the last number word used in a count represents the total number of objects in a set. The abstraction principle indicates that the objects that can be counted may be of any nature. Finally, the order irrelevance principle states that as long as the objects in a set are counted only once, the order in which the items are enumerated does not alter the result of the count.

In the present research verbal counting is defined as the child's ability to repeat the list of count words in the correct, pre-specified order. Cardinality is defined as children's understanding that the last word in a count represents the total amount of 
objects in a set. The present research assessed children's ability to map number words to quantities. The word-quantity mapping implies that children understand the semantic meaning of a number word. That is, they understand that the verbal number symbol represents a specific quantity (even if the physical set of objects is not present). In order to assess whether they have the mapping available, the tasks that were used in the present research presented the children with both verbal symbol and non-symbolic quantity representations. Children thus needed to know the meaning of the symbol and had to apply the cardinality principle to solve the task. Thus, listening to a verbal number and accessing the meaning is different from the counting principle of understanding that the last word in a count is the total number of objects in a set in the sense that word-quantity mapping is more a conceptual understanding of the symbols as representations of quantity.

\section{Written number representations}

Around the same time that children are learning the sequence of number words, they also begin learning to identify the Arabic digits (Mix, 2009). However, in order for children to further their understanding of more complex math, they must understand how the written number symbols represent quantity (e.g., Geary, Hoard, Nugent, \& Bailey 2013; Kolkman, Kroesbergen, \& Leseman, 2013; LeFevre, Fast, et al., 2010; Mundy \& Gilmore, 2009; Sowinski et al., 2015; Vanbist, Ceulemans, Ghesquière, \& DeSmedt, 2015).

Research has found that tasks that assess the ability to access quantity information from written symbolic number representations is related to children's calculation and word problem solving skills (Fuchs, Geary, et al., 2010; Geary, Bailey, \& Hoard, 2009; 
Jordan, Glutting, \& Ramineni, 2010). The ability to compare two symbolic number representations on the basis of magnitude is related to children's performance on arithmetic tasks both concurrently (e.g., Durand, Hulme, Larkin, \& Snowlin, 2005; Holloway \& Ansari, 2009) and longitudinally (Vanbist, Ansari, Ghesquière, \& De Smedt, 2016; Vanbist, Ceulemans, Ghesquière, \& DeSmedt, 2015; Vanbinst \& De Smedt, 2016). Furthermore, researchers have found that children with math learning difficulties show consistent difficulty in accessing quantity information from the written number representations (e.g., Defever, DeSmedt, \& Reynvoet, 2013; De Smedt \& Gilmore, 2011; Gersten, Jordan, \& Flojo, 2005; Iuculano, Tang, Hall, \& Butterworth, 2008; Rousselle \& Noël, 2007). The ability to access information about quantity from number symbols develops gradually as children integrate their knowledge about non-symbolic quantities and their knowledge of the number symbols. Several models that describe the development and integration of these early numeracy skills have been proposed.

\section{Models of early numeracy development}

In the following section I review three models of how early numeracy and mapping skills develop that lay the foundation for the present research. These models are (a) the Pathways model developed by LeFevre, Fast, et al. (2010), (b) the model of the proposed order of acquisition of the mappings by Bialystok (1992), and (c) the model of development of number representations by von Aster and Shalev (2007).

The Pathways Model. LeFevre, Fast, and colleagues (2010) developed and tested a model of early numeracy development that includes independent linguistic and quantitative cognitive precursors of children's mathematical skills. For example, children's linguistic skills (i.e., receptive vocabulary and phonological awareness) were 
related to the development of children's symbolic number skills, specifically, naming written numbers. Quantitative precursors (e.g., subitizing; which is the ability to rapidly enumerate sets of 1 to 3 dots) were related to children's ability to understand, manipulate, and operate on non-symbolic quantities. Both the linguistic and quantitative precursor skills were found to be significant predictors of more complex mathematical abilities such as calculation and magnitude comparison. Thus, findings by LeFevre and colleagues suggest that the development of advanced mathematical abilities requires the acquisition and integration of both linguistic/symbolic and quantitative precursor skills.

Model of the acquisition of the mappings. Bialystok (1992) provides an account of how young children integrate their quantitative and linguistic/symbolic skills. According to Bialystok, learning the associations among the three representations of number (i.e., quantities, number words, and written Arabic digits) is necessary for children to further their mathematical understanding. Bialystok proposed that children begin creating these associations or mappings by initially learning to identify each number symbol (i.e., number words and Arabic digits) and making the initial connection between them. That is, the initial mapping that children create is between number words and digits. According to Bialystok, children then learn to associate the number words to the quantities they represent and finally, children learn the mapping between digits and quantities. Furthermore, according to Bialystok, children can only develop more complex math skills (e.g., symbolic arithmetic) once they have acquired the mappings among the three representations of number, that is, once they have integrated their knowledge of the symbolic number representations and their quantitative knowledge. Consistent with Bialystok (1992), further studies have also found significant relations between the ability 
to associate or map among symbolic and non-symbolic number representations and performance on math assessments in children (e.g., Batchelor, Inglis, \& Gilmore, 2015; Brankaer, Ghesquière, \& DeSmedt, 2014; Purpura, Baroody, \& Lonigan, 2013; Sowinski, LeFevre, Skwarchuk, Kamawar, Bisanz, \& Smith-Chant, 2015) and adults (e.g., Castronovo \& Göbel, 2012). Thus, it is critical that children learn the associations among the number representations if they are to advance their math skills. However, the development of children's mapping skills requires prior knowledge of the number symbols and quantity which have also been proposed to develop sequentially.

Hierarchical model of numeracy development. Von Aster and Shalev (2007) developed a four-step model of number representation. According to their model, the first step is learning to determine the cardinal value of small sets, that is, subitizing. Children use this understanding of the meaning of the first few verbal number words to develop their knowledge of how larger non-symbolic quantities are associated with number words in the second step, and with Arabic digits in the third step. The fourth step in the model involves being able to map symbolic numbers onto a mental number line, implying that children understand the underlying quantity represented by the number symbols. According to von Aster and Shalev (2007), failure to acquire any of the skills involved in steps 1-3 would prevent children from successfully developing further math skills.

In summary, the models by LeFevre, Fast, et al. (2010) and von Aster and Shalev (2007) both propose that children's initial linguistic/symbolic and quantitative precursor skills need to be integrated in order for them to advance their knowledge of math. Bialystok's model of the development of the mappings may provide the possible stepping stones through which this integration of the linguistic and quantitative precursor 
pathways occurs: a) children's linguistic skills are required for children to develop the mapping between number words and digits; b) children's ability to map number words to quantities requires them to have an understanding of quantity, thus both the linguistic/symbolic and quantitative pathways contribute to the development of children's mapping between number words and digits and number words and quantities which then, according to Bialystok, are necessary to develop the mapping between digits and quantities. These mappings will allow children to both encode and retrieve quantity information to and from number symbols.

\section{Children's mapping skills}

An increasing number of studies have been conducted on children's early mapping skills (e.g., Batchelor, Keeble \& Gilmore, 2015; Benoit et al., 2013; Knudsen et al., 2015; Mundy \& Gilmore, 2009). Researchers have addressed multiple issues such as: a) the relation between mapping skills and early numeracy skills such as counting, cardinality (Batchelor, Keeble, \& Gilmore, 2015) and later math skills such as symbolic number comparison (Mundy \& Gilmore, 2009); b) whether children learn to map in one direction (e.g., number words to quantities) prior to the other (quantities to number words) (e.g., Odic, LeCorre, \& Halberda, 2015); and c) the order of acquisition of the mappings among the three representations of number (e.g., Benoit et al., 2013). Results across studies have been inconsistent. In the following section I provide an overview of the research that has addressed the various issues related to children's mapping skills.

Children's mapping in relation to counting. One of the first studies to examine children's mapping between number words and quantities and children's knowledge of the verbal count list was conducted by Lipton and Spelke (2005). The researchers 
assessed five-year-old children's verbal counting skill and performance on an estimation task that included large numerosities (i.e., 20-120). Lipton and Spelke's results showed a positive relation between verbal counting and estimation skill: children were able to provide accurate estimates of the numerosities that were within their counting range.

Batchelor, Keeble, and Gilmore (2015) further investigated the relationship between verbal counting, and children's ability to map number words to non-symbolic quantities for two- to four-year-old children; importantly, the researchers also assessed the relation between word-quantity mapping and children's understanding of cardinality. The authors assessed two-to-four-year-old-children on verbal counting, cardinality, and a word-quantity mapping task. In the mapping task, children were shown a non-symbolic quantity and a verbal number word and were asked to determine which represented the larger number (numbers ranged from 1 to 10 and children were not allowed to count). Batchelor et al. found that children's cardinality but not their verbal counting skill was predictive of their performance on the mapping task. In addition, Batchelor, Keeble, and Gilmore found that children who had only learned the meaning of "three" or "four" (but had not yet acquired full understanding of the cardinal principle) performed above chance only on the trials within the range of numbers for which they knew the cardinal meaning. Further analyses however, revealed that some children who had not yet acquired the cardinal principle were able to succeed on the mapping task. The authors concluded that while some children develop the word-quantity mapping after acquiring the cardinality principle, for other children the development of the word-quantity mappings may play a role in the acquisition of cardinality. Thus, the relation between counting, cardinality, and children's mapping skills requires further research. 
Children's mapping skills and more advanced math. Children's ability to understand how the number symbols are used to represent quantity is related to the development of their more complex mathematical skills. For example, Mundy and Gilmore (2009; Study 2) found that six-to-eight-year-old children's ability to map between symbolic (written and verbal) and non-symbolic number representations significantly predicted performance on a school mathematics test. Batchelor, Inglis, and Gilmore (2015) found that four-to-five-year-old children's ability to determine the digit that corresponded to a given non-symbolic quantity was predictive of performance on a test of early arithmetic and a symbolic number comparison task. Thus, children's ability to associate the symbolic number representations to their non-symbolic quantity is related to the development of further mathematical skills.

Direction of mapping. Researchers have also examined whether children learn to map between two number representations in both directions at the same time or whether one direction (e.g., digit to quantity) develops prior to the other (quantity to digit). Mundy and Gilmore (2009; Study 1) assessed the development of children's symbol-to-quantity mapping in six- and eight-year-old-children, and whether children could map in both directions (i.e., from symbol to quantity, and from quantity to symbol). The researchers developed two novel tasks in which children had to match a) a target digit (the corresponding verbal label was provided simultaneously) to the corresponding nonsymbolic representation and, b) a target non-symbolic quantity to the corresponding digit. Children were always given two options from which to select the answer. The target quantities and symbolic representations ranged from 20 to 50 and differed in ratios of .67 (e.g., 20 vs. 30) and .50 (e.g., 21 vs. 42). Mundy and Gilmore found that eight-year-olds 
performed better than six-year-olds, indicating that the ability to map between number representations continues to develop in children older than six. Children performed equally well on both tasks at the .50 ratio but found it more difficult to map from symbolic to non-symbolic representations than to map non-symbolic representations to digits at the .67 ratio. Mundy and Gilmore concluded that the pattern of performance was due to the approximate nature of their task: children may have found it more difficult to map from a symbol to quantity than from quantity to symbol for the .67 ratio because when mapping from a symbol to the quantity children were presented with two nonsymbolic quantities for which the distance between them was smaller (i.e., .67 ratio as opposed to .50 where no effect of direction was found). The smaller distance between the non-symbolic quantities may have increased the difficulty in discriminating between the quantities making the symbol-quantity task harder than the quantity-symbol task in which children did not need to discriminate between two non-symbolic quantity representations (i.e., the two response options were symbolic).

Following Mundy and Gilmore (2009), Brankaer et al. (2014) also investigated digit-quantity mapping in six- and eight-year-old-children, however, the mapping tasks used by Brankaer et al. assessed only the numbers 1-9. Consistent with Mundy and Gilmore, the findings showed that eight-year-olds performed significantly better than sixyear-olds; however, unlike Mundy and Gilmore, the authors found no difference in performance between digit-quantity and quantity-digit mapping tasks. The authors attributed this difference to the fact that smaller quantities were used and thus the tasks were less approximate in nature. 
The studies by Mundy and Gilmore (2009) and Brankaer et al. (2014) only examined the mapping between digits (and number words) and quantities and included only elementary school-aged children. Odic, LeCorre, and Halberda (2015) examined whether, for two-to-five-year-old children, the mappings from number words to quantities and quantities to number words develop at the same time. The word-toquantity mapping was assessed with a task in which the experimenter asked the child to "pat as fast as he/she could" a puppet on the head a specific number of times within the range of 1 to 10 . The quantity-to-word mapping was assessed using the task known as "Fast Cards" (LeCorre \& Carey, 2007). On this task, children were shown a card with 1 to 10 dots and asked to name the number of dots without counting. The authors assessed children's mappings in relation to their cardinality skill and found that both children who had not yet developed a full understanding of the cardinal principle (i.e., two or three knowers) and those who had already mastered the cardinality principle successfully mapped number words to quantities, but not quantities to number words. The authors concluded that children develop the mapping from number words to quantities prior to mapping from quantities to number words.

Mundy and Gilmore (2009), Brankaer et al. (2014) and Odic et al. (2015) assessed the effect of mapping direction only on symbol-quantity mappings. In contrast, Benoit et al. (2013) tested for effects of direction of mapping on all three mappings (i.e., words and digits, words and quantities, and digits and quantities). Accordingly, because they included all three mappings, the researchers also investigated the order in which children acquire the mappings among the three number representations. 
Order of acquisition of the mappings. Benoit et al. (2013) assessed three-, four-, and five-year-old children on six mapping tasks using the numbers 1 to 6 (i.e., numberword-to-quantity, quantity-to-number-word, number-word-to-digit, digit-to-numberword, digit-to-quantity, quantity-to-digit). Their goal was to test whether the direction of the mapping was related to children's performance and to assess the order in which children acquire the mappings among number representations with small numbers. Benoit et al. did not find effects of the direction of mapping on children's performance on any of the six tasks; and by age five, children performed at ceiling on all tasks. With respect to the order of acquisition of the mappings, their analyses showed that children develop the mapping between number words and quantities and between digits and quantities prior to developing the between number words and digits. Their conclusions stood in sharp contrast with the proposal by Bialystok (1992) and with prior research by Mix (2009) who showed that children begin to develop the link between verbal and written number representations at around the age of two. The authors suggested that children's experience with the visual number representations in school could account for the observed pattern of development.

In contrast to Benoit et al. (2013) but consistent with the predictions of Bialystok (1992), Knudsen et al. (2015) found evidence that the last mapping to develop is that between digits and quantities. The authors investigated the developmental sequence of children's acquisition of the mappings between digits and non-symbolic quantities by assessing four-, five-, six-, and seven-year-old children on four tasks in which they needed to apply their knowledge of the quantities underlying the digits 1 to 6 . In contrast to previous studies of mapping skills that used matching tasks, Knudsen and colleagues 
employed several different types of tasks, including digit naming, digit sorting (i.e., children had to arrange cards with the digits in the correct order), Arabic digit Give-N (i.e., children were shown a digit and asked to provide the corresponding number of objects), and verbal number word Give-N tasks (i.e., when children failed to produce the correct number of objects after being shown a digit, they were asked to provide the amount of objects that corresponded to the verbal number word). Six- and seven-yearolds performed at ceiling on all tasks. The authors found that four-year-olds performed worse than five-year-olds but that a consistent pattern of performance emerged in both groups: performance was better on the Give $\mathrm{N}$ with number words, followed by digit naming, and performance was poorer on the Give $\mathrm{N}$ task with Arabic digits and worst on the digit sorting task. Thus, consistent with the proposal by Bialystok (1992), Knudsen et al. concluded that children develop the mapping between digits and quantities after having acquired the mappings between number words and digits and number words and quantities.

\section{Summary}

Research on children's mapping skills has provided evidence that a) the mappings among the different number representations begin developing during the preschool years (Benoit et al., 2013; Carey, 2004; Fuson, 1988; Knudsen et al., 2015; Mix, 2009; Wynn, 1990, 1992), b) that the mapping abilities continue to develop through middle childhood (Brankaer et al., 2014; Mundy \& Gilmore, 2009), c) that children's mapping skills are related to the development of further more complex math skills (Batchelor, Inglis, \& Gilmore, 2015; Mundy \& Gilmore, 2009); and that the mappings among the three number representations do not develop at the same time (Benoit et al., 2013; Knudsen et 
al., 2015). However, the current literature has not settled the question of whether cardinality knowledge precedes children's acquisition of the mappings (Batchelor et al., 2015a) and has provided contrasting results with regards to whether children learn to map in one direction prior to the other (e.g., Benoit et al., 2013; Brankaer et al., 2014; Mundy \& Gilmore, 2009; Odic, LeCorre, \& Halberda, 2015) or whether children learn to map in both directions at the same time (e.g., Benoit et al., 2013).

Contrasting results have also been found with regards to the order of acquisition of the mappings (e.g., Benoit et al., 2013; Knudsen et al., 2015). The contrasting patterns of development found by Benoit et al. (2013) and Knudsen et al. (2015) could reflect a) differences in the methods chosen to assess the mappings, b) as suggested by Benoit et al., differences in children's exposure to different educational experiences, or c) overall cultural differences in home numeracy practices as the studies were conducted in different countries.

In Chapter 3, I assess the children's mapping skills in relation to skills which are assumed to function as precursors to the mappings, specifically, children's verbal counting skill, children's ability to understand and manipulate non-symbolic quantities, and children's ability to identify verbal and written number representations. I also assess whether children's mapping skills are predictive of their ability to compare two symbolic number representations. 


\section{CHAPTER 3: STUDY 1}

The paper included in this chapter has been resubmitted to the journal Cognition after a revise/submit decision. The current version is included as it was submitted.

The integration of symbolic and non-symbolic representations of exact quantity predicts number comparison performance in preschool children

Carolina Jiménez Lira, Miranda Carver, Heather Douglas, and Jo-Anne LeFevre Carleton University

Author note ${ }^{1}$.

Carolina Jiménez Lira, Miranda Carver, and Jo-Anne LeFevre, Department of Psychology; Heather Douglas and Jo-Anne LeFevre, Institute of Cognitive Science.

This research was supported by a grant from the Natural Sciences and Engineering Research Council of Canada to Jo-Anne LeFevre and a scholarship from CONACyT (the National Council of Science and Technology of Mexico) to Carolina Jiménez Lira.

Correspondence concerning this article should be sent to Jo-Anne LeFevre, Institute of Cognitive Science, Carleton University, 1125 Colonel By Drive, Ottawa, K1S 5B6, Canada or via the internet to jo-anne.lefevre@carleton.ca.

\footnotetext{
${ }^{1}$ The authors contributed to the completion of the manuscript in the following way: Carolina Jiménez Lira: data collection, analysis, and write up of the manuscript. Miranda Carver and Heather Douglas: data collection, revision of the manuscript. Jo-Anne LeFevre: data analyses and write up of the manuscript.
} 


\begin{abstract}
Preschoolers $(n=62)$ completed tasks that tapped their knowledge of symbolic and nonsymbolic exact quantities, their ability to translate among different representations of exact quantity (i.e., digits, number words, and non-symbolic quantities), and their nonsymbolic, digit, and spoken number comparison skills (e.g., which is larger, 2 or 4?). As hypothesized, children's knowledge about non-symbolic exact quantities, spoken number words, and digits predicted their ability to map between symbolic and non-symbolic exact quantities. Further, their knowledge of the mappings between digits and nonsymbolic quantities predicted symbolic number comparison (i.e., of spoken number words or written digits). Mappings between written digits and non-symbolic exact quantities developed later than the other mappings. These results support a model of early number knowledge in which integration across symbolic and non-symbolic representations of exact quantity underlies the development of children's number comparison skills.
\end{abstract}

Abstract: 138 words Keywords: early number skills; cardinality; symbolic number comparison; preschoolers; small exact quantities; mapping digits to quantities 


\section{Introduction}

Children need to understand the symbolic number system before they can progress in other mathematical tasks that involve the use of symbols (e.g., arithmetic, measurement, fractions). Accordingly, children must learn the mappings among non-symbolic quantities (e.g., sets of objects), spoken number words, and written digits (Bialystok, 1992; Purpura, Baroody, \& Lonigan, 2013; von Aster \& Shalev, 2007; see Figure 3.1). Despite the importance of knowing the symbolic system, however, few studies have explored how young children develop the connections among the three representations (Knudsen, Fischer, Henning, \& Aschersleben, 2015). Accordingly, we explored several questions. First, we hypothesized that knowledge about number words, exact quantities, and digits as categories of representations would predict preschool children's learning about the mappings among these representations. Second, we hypothesized that learning the mapping between written digits (e.g., '3') and exact quantities (e.g., ***) would depend on children first learning the mapping between spoken number words and exact quantities (/Ori/ and ***) and number words and digits (/Ori/ and '3'). Third, we evaluated a model of how knowledge of representations and of the mappings among these representations predicts symbolic and non-symbolic number comparison performance. Number comparison performance with symbolic digits (e.g., which is larger, 2 or 7?) predicts children's arithmetic knowledge in the early elementary grades (DeSmedt, Noël, Gilmore, \& Ansari, 2013; DeSmedt, Verschaffel \& Ghesquire, 2009; Holloway \& Ansari, 2009; Lyons, Price, Vaessen, Blomert, \& Ansari, 2014) and thus is an important aspect of children's early mathematical learning. 


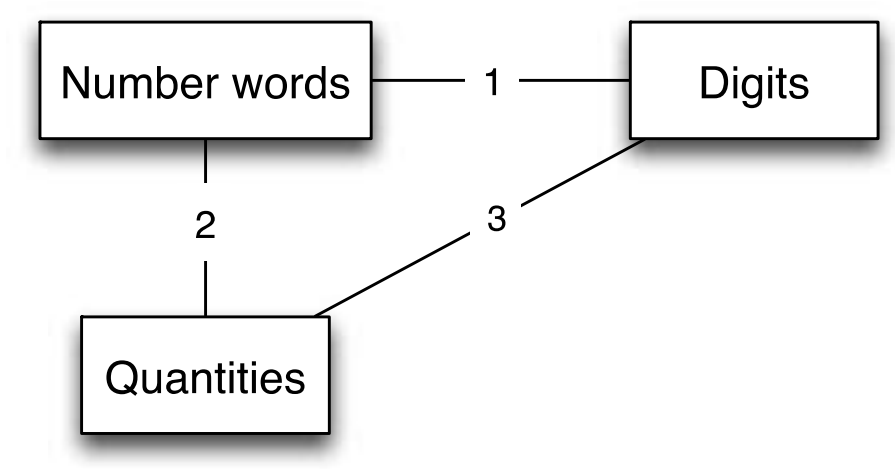

Figure 3.1. Mappings among the three representations of number.

Quantities are groups of objects and thus are non-symbolic; number words refer to the spoken number words; digits refer to the written Arabic digits (e.g., 2, 4, 9). The numbers on the links represent the hypothesized order of acquisition of these mappings according to Bialystok (1992) and von Aster and Shalev (2007) such that links 1 and 2 are acquired concurrently, and 3 develops later.

\subsection{Learning the mappings among quantities, number words, and digits}

Children can recognize and discriminate small exact quantities from an early age (Le Corre \& Carey, 2007) and they share this ability with other vertebrates (Ansari, 2008; Agrillo, Piffer, Bisazza, \& Butterworth, 2012; Range, Jenikejew, Schröder, \&Virányi, 2014). ${ }^{2}$ Despite this early competence, the mappings between small exact quantities and the associated number words develop slowly (Wynn, 1990, 1992). Children start learning spoken number words early and most three-year-olds know the counting words to ten as a verbal string before they can recognize the digits to 10 (e.g., Mix, 2002, 2009). By age 4,

\footnotetext{
${ }^{2}$ Human also have access to a non-symbolic approximate magnitude system that is shared with other animals and is used to process large quantities (Ansari, 2008). The role of the approximate magnitude system is not addressed in the present research. There is strong evidence for the importance of symbolic number knowledge for mathematical development but the role of the approximate magnitude system is less clear (see De Smedt et al., 2013 for a review).
} 
many children have acquired understanding of the cardinal principle for sets up to 5 or 6 , that is, they understand that the last number word in a counting sequence represents the quantity of that set (Le Corre, Van de Walle, Brannon \& Carey, 2006; Le Corre \& Carey, 2007; Sarnecka \& Carey, 2008; Shusterman, Gibson \& Finder, 2010). Mapping the digits to the quantities, however, appears to develop later, although only a few studies have explored the development of quantity-digit mapping directly (Knudsen et al., 2015). Mapping between written symbols and their meaning (i.e., quantities) underlies tasks such as magnitude comparison and symbolic arithmetic. Accordingly, the use of written symbols predicts arithmetic skill several years later (Zhang, Koponen, Räsänen, Aunola, Lerkkanen, \& Nurmi, 2014) and this link may be mediated by counting sequence knowledge (see also Purpura et al., 2013) and other numerical language skills (Moll, Snowling, Göbel, \& Hulme, 2015). These studies suggest that the mappings between written symbolic numbers and exact quantities are central to children's developing numeracy skill.

Despite the importance of knowing the symbolic system, however, few studies have directly assessed the order in which preschool children (i.e., two-to-four-year olds) make the connections among the three representations (cf. Batchelor, Keeble, \& Gilmore, 2015; Benoit, Lehalle, Molina, Tijus, \& Jouen, 2013; Odic, Le Corre, \& Halberda, 2015; Knudsen et al., 2015). Although most existing evidence is consistent with the view that digit-quantity mapping develops last (Bialystok, 1992; Carey, 2004; Fuson, 1988;

Knudsen et al., 2015; Soltësz, Szücs, \& Szücs, 2010; Zhou \& Wang, 2004), only one previous study tested children's knowledge of the mappings in both directions among all three representations. Benoit et al. (2013) asked 3-, 4-, and 5-year-old children to perform 
six mapping tasks, translating between quantities, number names (i.e., spoken number words), and written digits in both directions, for both small (i.e., 1, 2 and 3) and larger numbers (i.e., 4, 5, and 6). Benoit et al. concluded that children learn to map quantities to number words and quantities to digits before they can directly connect number names to digits. This order of acquisition would be represented in Figure 3.1 as learning pathways 2 and 3 before pathway 1 .

In contrast to Benoit et al. (2013), Bialystok (1992) proposed that children first learn the sequence of count words as a verbal string, then learn to associate each count word with a written digit, and finally, learn to map the digit to the quantity (Bialystok, 1992; Bialystok \& Codd, 1996, 2000), represented in Figure 3.1 as learning pathways 1 and 2 before pathway 3 . According to Bialystok, children learn the digit-to-quantity connection last because this link implies that they have a mental representation of the number concept. To build this mental representation, children derive the understanding of the relations between written numbers and quantities from their knowledge that each number word can be mapped onto a specific digit and that each number word represents a unique quantity. Thus, understanding the concept of cardinality, in this view, is a necessary predecessor of mapping digits to quantities. Similarly, Knudsen et al. (2015) found that children know the cardinal meanings of the number words before they map digits to quantities, although they suggested that mapping number words to digits is learned concurrently with digit-quantity mapping, that is, learning pathway 2 first, followed by 1 and 3 together.

In contrast, to explain why children in their study learned to map digits to number words last (i.e., pathway 1), Benoit et al. reasoned that children find it easier to map 
between non-symbolic and symbolic representations (that is, quantities to number words or quantities to digits) than between two symbolic representations (that is, digits and number words). They also speculated that because both quantities and digits are visual and thus continuously available, it might be easier for children to connect these two representations than to map auditory number words to either digits or quantities. In summary, although research suggests that children learn the mappings between spoken number words and quantities before they map written digits to quantities, the specific order in which the pathways are integrated has not been fully described.

\subsection{Present Study: Assessing Children's Mapping across Number Representations}

In the present research we explored the order in which children acquire the mappings among quantities, spoken number words, and written digits within the framework of the models developed by Bialystok (1992) and von Aster \& Shalev (2007). The predictions with regards to the order of acquisition of the mappings were based on prior research (Bialystok, 1992; Mix, 2009). First, we hypothesized that children would initially learn to associate number words to digits because this mapping requires only that children assign a verbal label to a written form. Furthermore, digit-word mapping may not be strongly related to the size of the numbers because these mappings can be learned without understanding the quantities involved (i.e., by rote). Second, because the mappings between number words and quantities require the more complex understanding of how the verbal counting string is used to represent quantity, this mapping was hypothesized to develop after word-digit mapping and be linked to children's developing counting skills. Performance on word-quantity mapping will be related to number size, because counting is more difficult and error-prone for larger numbers. Finally, we 
hypothesized that children would develop the mapping between digits and quantities once the mappings between number words and digits and number words and quantities were in place (e.g., Bialystok, 1992). Furthermore, we used the pathways of children's numeracy development proposed by LeFevre et al. (2010) to integrate the mapping abilities into a comprehensive model. Specifically, we investigated children's mappings among the three representations (quantities, number words, and digits) using modified versions of the tasks used by Benoit et al. (2013). All children performed all six possible mapping tasks. The mapping tasks were equated for task difficulty and each mapping task required a two-choice recognition decision. This design allowed us to test whether children were able to map in both directions between representations (cf. Benoit et al., 2013; Odic et al., 2015). We also used a completely within-groups design for evaluating the order in which specific pathways developed. ${ }^{3}$

\subsection{Predictors of Number Comparison and Precursors of Mapping}

Symbolic number comparison tasks require children to choose the larger (or smaller) quantities represented by digits or number words, and thus presuppose a mapping process between the symbol (word or digit) and a mental representation of quantity. Older children show consistent correlations between performance on symbolic number comparison tasks and mathematical knowledge (Holloway \& Ansari, 2008, 2009; Lyons, et al., 2014). We hypothesized, therefore, that performance on the verbal and digit

\footnotetext{
${ }^{3}$ Throughout the remainder of the paper, when we refer to a specific mapping direction, we will indicate that by including 'to' in the label (e.g., number-word-to-quantity mapping or digit-to-number-word mapping). In contrast, when we are referring to the pathway, independently of the direction of mapping, we will hyphenate the labels (e.g., number word-quantity path or digit-number word mapping).
} 
comparison tasks would be predicted by children's ability to map among all three representations (cf. Batchelor et al., 2015).

Figure 3.2 shows our hypothesized model of the inter-relations among the precursor skills and the mapping tasks, and how these may be related to performance on number comparison. As shown in Figure 3.2, we hypothesized that quantity-digit mapping was acquired last and reflects the integration across the three representations and therefore that performance on the quantity-digit task would mediate the relations between the other mapping skills and symbolic comparison performance. The figure also shows the skills assumed to be precursor abilities for mapping, including knowledge of the verbal counting sequence (number names and their order), as well as knowledge of number words and digits as specific categories of spoken and written symbols ("symbol knowledge" in the figure). Further, the ability to physically and mentally represent and manipulate quantities was expected to predict mapping of number words to quantities. Consistent with the model of early numeracy development proposed by LeFevre et al. (2010), the upper portion of Figure 3.2 represents the linguistic/symbolic pathway and the lower portion represents the quantitative pathway. We propose an extension to that model which shows that these pathways are integrated in the mapping of digits to quantities. 


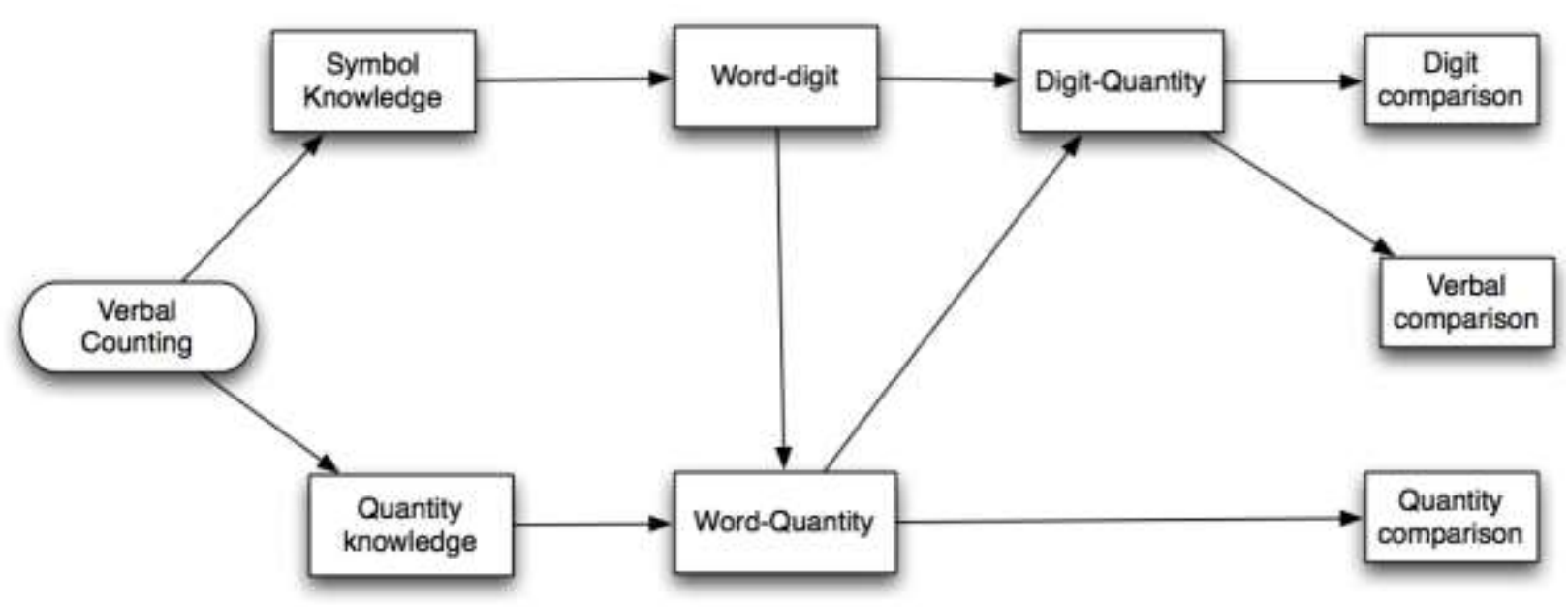

Figure 3.2. Hypothesized relations among precursor, mapping and comparison skills.

The skills in the top row are part of the symbolic/linguistic pathway whereas the skills in the bottom row are part of the quantitative pathway (LeFevre et al., 2010).

In summary, the proposed model extends the Pathways framework by postulating that the symbolic and quantitative pathways merge when children integrate their knowledge of written symbols and quantities (i.e., when they can directly link digits to quantities; Bialystok, 1992; Knudsen et al., 2015). In contrast, non-symbolic comparison performance, an ability that children have very early, is separate from the symbolic pathway, according to this model. In summary, Figure 3.2 captures our hypotheses about the relations among the various components of early numeracy knowledge.

\section{Method}

\subsection{Participants}

Sixty-eight two-to-four-year-old children were recruited from daycares in a large Canadian city. Six of the children were excluded from the present analyses because they were missing data on at least one of the tasks. The remaining 62 children ( 28 girls) had a mean age of three years and four months $(M=40.4$ months, $S D=5.5$, range 25 to 51 
months $\left.{ }^{4}\right)$. Permission to conduct testing was obtained from the daycare directors and from parents. Before testing, children were asked whether or not they wished to participate and they were permitted to stop the testing at any time.

The language used in the daycares was English. Forty of the children were monolingual English speakers. Nineteen children spoke a second language in addition to English at home (i.e., Amharic, French, Hungarian, German, Italian, Mandarin, Pashto Dari, Russian, Spanish, Tamil, Ukrainian, or Portuguese), and three children spoke two languages in addition to English (Ukrainian and French, Ukrainian and Russian, French and Hungarian). Of the 57 mothers who provided information about their highest level of education, $4 \%$ had less than high school, $18 \%$ had a high school diploma, $51 \%$ had a college or university degree, and $28 \%$ had a post graduate degree. Fathers had similar levels of education as mothers. These families were thus typical of the diversity found in Canadian cities, with generally middle-class status.

\subsection{Measures}

Children attempted 15 different tasks. These tasks fell into three categories: (a) knowledge of representations (six tasks), (b) mapping between representations (six tasks), and (c) comparisons (three tasks). The tasks are listed in Table 3.1, with descriptive statistics, sessions in which the tasks were administered, number of trials, maximum time, and reliability. The tasks were presented in a fixed order. All the mapping tasks were presented in Session 2. The order of tasks in Session 1 was: number word versus word, verbal counting, knowledge of quantity (visible then hidden), quantity comparison, digits versus letters, digits versus quantities, verbal comparison, and digit comparison. For

\footnotetext{
${ }^{4}$ The frequency distribution for the age of the participants is presented in Appendix A.
} 
Session 2 the order of tasks was: quantity-to-number-word, number-word-to-quantity, number-word-to-digit, digit-to-number-word, quantity-to-digit, digit-to-quantity. In most tasks, numbers from one to nine were used, with 1 to 3 categorized as small, 4 to 6 categorized as medium, and 7 to 9 categorized as large. Children were always given trials from the small number set first, followed by the medium set, and then the large set. Puppets were used in some tasks to engage the children. Examples of how the mapping tasks were set up for the children are shown in Figure 3.3. 


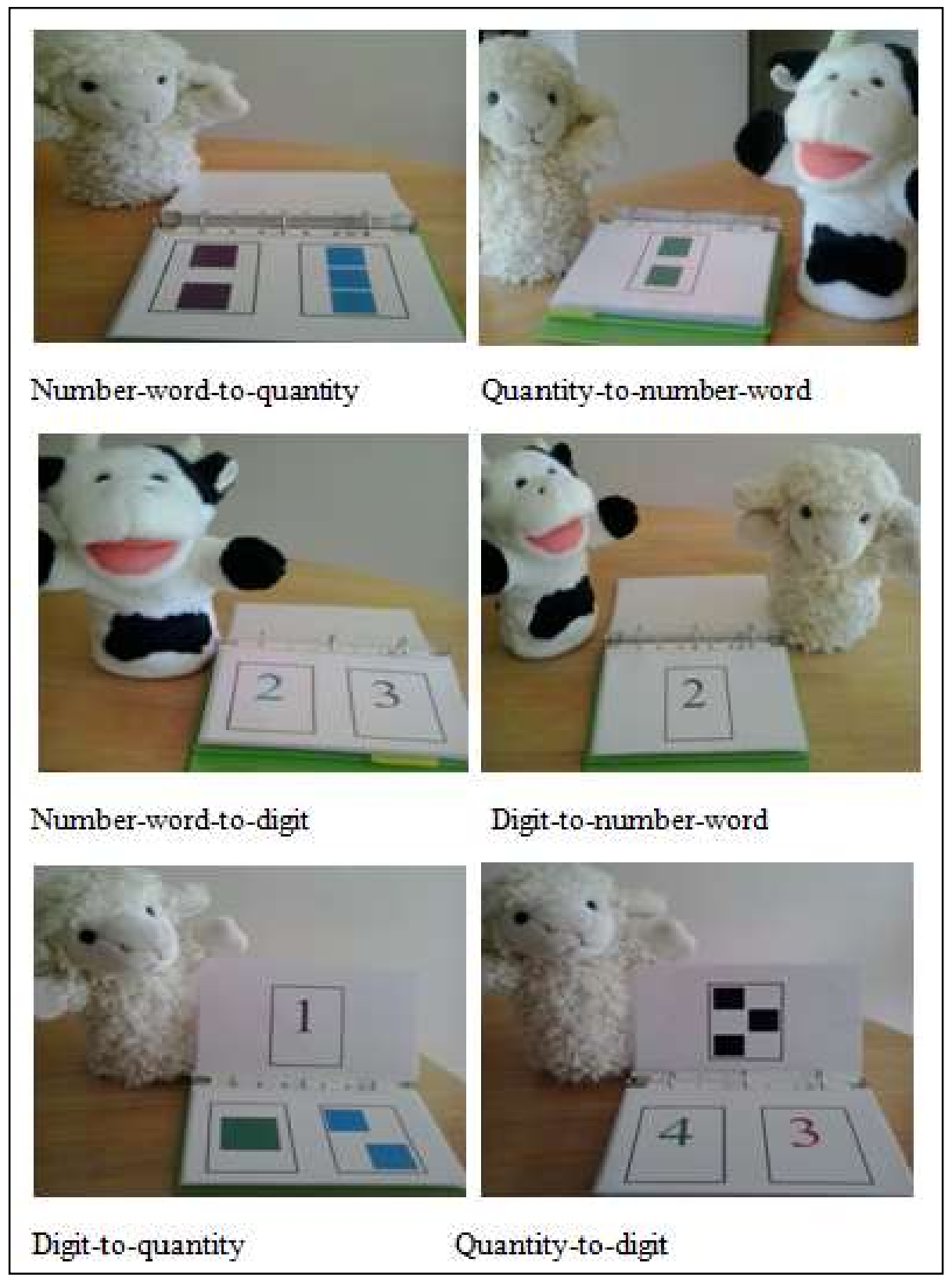

Figure 3.3. Examples of how the mapping tasks appeared to the children.

For the quantity-to-number-word and digit-to-number-word tasks, the puppets spoke the targets and the child chose the puppet who had said the correct number word. 
Table 3.1. Performance on all tasks (reliability is Cronbach's alpha).

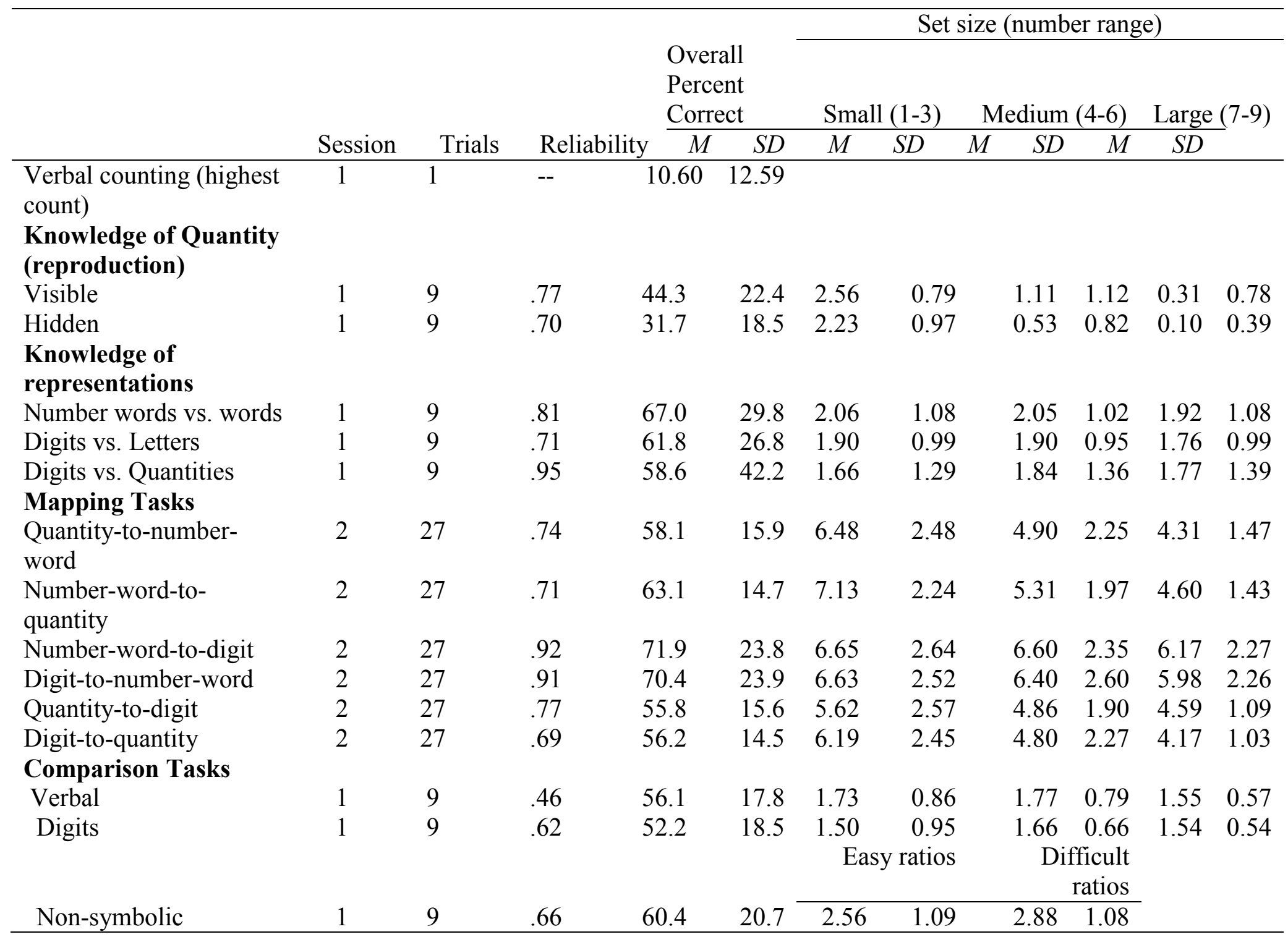




\subsubsection{Knowledge of Representations}

\subsubsection{Verbal counting}

Holding a puppet, the experimenter said to the child: "We are going to play with Belle! She wants to learn how to count, can you help her? She can only count to one! Listen," The experimenter then said "One." To then prompt the child to begin counting the puppet continued: "What comes after one? Can you help me count?" If the child did not begin counting the experimenter said: "Do you know what number comes after one? Let's see: one, two... what comes after two?" The score on this task was the number up to which the child could count without mistakes. If the child recognized and corrected his/ her mistake it was scored as correct. Children were not stopped by the experimenter.

\subsubsection{Quantity Knowledge}

Children were given two tasks in which they reproduced non-symbolic quantities, one involving visual arrays (Levine, Jordan \& Huttenlocher, 1992; Negen \& Sarnecka, 2009) and the other with hidden arrays. Materials for these tasks included 12 green and 12 blue foam cubes (approximately $3 \mathrm{~cm}$ high), two place mats, and an opaque plastic panel (for the hidden quantity version). Before starting each task, the experimenter asked the child to choose one set of cubes. Both versions of the task included 9 trials assessing the numbers 1-9; children received 1 point for every correct response. For both quantity knowledge tasks, children were presented with the small number set (i.e., 1-3) first, followed by the medium (i.e., 4-6), and large (i.e., 7-9); the trials within each set size was presented in random order. Feedback as to the correctness of the response was not provided. The tasks ended when the child failed to reproduce two consecutive sets of objects. 
For the visual reproduction task, the experimenter told the child to watch as she placed some cubes on the mat. Then the experimenter asked the child to put the same amount of cubes on his or her mat. Number words were never used throughout the trials. In the hidden set version of this task, the experimenter placed a set of cubes on the mat, allowed the child to see it and asked "OK, have you seen the cubes?" Then using the opaque panel the cubes were hidden from the child's view. Then the experimenter said to the child: "Now can you place the same amount of cubes as I have on your mat?"

\subsubsection{Symbol Knowledge}

Digits versus letters. The purpose of this task was to assess the number of Arabic digits children were able to visually identify. As shown in Table 3.2, the pairs of numbers and letters were chosen because they have similar shapes. Materials for this task included a puppet and a binder $(19 \times 23 \mathrm{~cm})$ that contained white paper cards $(14 \times 21 \mathrm{~cm})$ with the stimuli printed and placed within a protective transparent plastic cover. On the cards, the pairs were enclosed within rectangles $(7 \times 10 \mathrm{~cm})$ separated by a $3 \mathrm{~cm}$ space. The letters and/or digits were printed in different colors (blue, green, red or purple; Times New Roman font size 170).

The experimenter explained: "We are going to play with Belle (the cow puppet)! In this game, you have to help Belle pick the numbers. I will show you some cards and when you see a number, you have to point to it." The experimenter then proceeded to show the child the cards that showed a digit on one side and a letter on the other, without mentioning any specific number words. The child saw all digits 1-9 in ascending order and the position of the digit was counterbalanced across trials. Children received 1 point for every correct response. 
Number words vs. words. The purpose of this task was to assess how many number words the participants could identify. The stimuli used for this task are shown in Table 3.2. The words were chosen to sound similar to the number and so that the children could not infer quantity or make a singular-plural distinction using the word (Condry \& Spelke, 2008). The experimenter introduced this task by placing the puppets on the table and telling the child that they were going to play a game with the sheep puppet Dolly and the cow puppet Belle. "In this game, Dolly will say a word and Belle will say a word, you have to listen carefully and then point to the puppet that says a number." The experimenter then lifted one of the puppets and said either a number or a similar sounding word, then she put the puppet down and lifted the other and said a number or a similar sounding word. The order in which children heard the number and the word were randomized across the trials. Children received 1 point for each correct response.

Table 3.2. Stimuli for the tasks requiring children to select between representations

\begin{tabular}{lccccccccc}
\hline \multicolumn{8}{c}{ Digits versus Letters } \\
\hline Digit & 1 & 2 & 3 & 4 & 5 & 6 & 7 & 8 & 9 \\
Letter & $\mathrm{T}$ & $\mathrm{Z}$ & $\mathrm{E}$ & $\mathrm{A}$ & $\mathrm{S}$ & $\mathrm{G}$ & $\mathrm{F}$ & $\mathrm{B}$ & $\mathrm{P}$ \\
\hline \multicolumn{7}{c}{ Number words versus control words } \\
\hline Number & One & Two & Three & Four & Five & Six & Seven & Eight & Nine \\
Control & Fun & True & Me & More & Dive & Fix & Ellen & Late & Shine \\
\hline
\end{tabular}

Digits versus quantities. The purpose of this task was to assess children's understanding that digits can be used to efficiently communicate quantity (Bialystok \& Codd, 1996). Materials for this task included a puppet, a binder with a set of 9 cards with two rectangles, one containing a colored digit in the range of 1-9, and the other containing an analog representation of quantity (i.e., circles -same color as the digit- 1.9 
$\mathrm{cm}$ in diameter) in the range of 1 to 9 printed within the other, and a set of 4 small plastic boxes (approximately $5 \times 3 \times 3 \mathrm{~cm}$ ); the boxes were of different colors: red, blue, green, and purple. The color of the box shown to the child on each trial matched the color of the digits and analog representations shown in the binder.

The experimenter introduced the task by saying "In this game, we need to help Dolly the Sheep. She needs to write a note to her friend to tell her that she has some toys inside this small box," the experimenter showed one of the boxes to the child; next the experimenter showed the child the first comparison card and said to the child: "the problem is that Dolly doesn't know how she should write numbers, can you point to how Dolly should write the number of toys in the box?"

We wished to assess only whether the child understood that Arabic digits are used to denote quantity (therefore there were no actual toys in the boxes); if the child understood this property of the Arabic digits, he/she would choose the digit on all occasions regardless of the quantity it denoted. Note that choosing the quantity representation was not an incorrect answer, however denoting quantities in a nonsymbolic format is considered less efficient than denoting them with a written number symbol. All the digits 1 through 9 and their corresponding analog representations were presented to the child in ascending order with the position of the digit counterbalanced across trials. Children received 1 point for choosing the digit as a more effective way of denoting quantity.

\subsubsection{Mapping}

Children completed 6 matching tasks: a) quantity-to-number-word, b) numberword-to-quantity, c) number-word-to-digit, d) digit-to-number-word, e) quantity-to-digit, 
and f) digit-to-quantity. On these tasks, children had to choose which of two stimuli matched a target stimulus. For example, for quantity-to-digit mapping, the child was presented with the target quantity (i.e., a card with 3 squares printed on it) and was asked to choose between two Arabic digits (e.g., 4 and 3) (see Figure 3.3). The child was presented with three trials for each target number (i.e., 1 through 9). For example, when assessing the mapping between the non-symbolic quantity “ $\square$ " and the written representation "2", the child was presented with a card with 2 squares printed on it, the three trials then consisted of presenting the child with the following pairs of Arabic digits: a) 2 and 1, b) 3 and 2, and c) 4 and 2. To keep the six matching tasks as similar as possible, the same comparison pairs were used for each target number across tasks. These are listed in Appendix B.

During the matching tasks, the child was not instructed to count but was allowed to do so if he/she wished to. The six matching tasks included a single practice trial in which the target number was 1 and the response options consisted of the numbers 1 and 9; feedback as to the correctness of the answer was only provided for this practice trial. Having used number one in the practice trial all matching tasks began with the target number two, and then proceeded with number one and finally numbers 3-9. Materials for these tasks included puppets and binders containing the stimuli, following the dimensions and format mentioned previously.

When the comparison pairs were non-symbolic sets, these were composed of two different color squares (blue, green, red, or purple) and placed within the two rectangles. The area of the squares was negatively correlated with the number of squares within the set. The sizes of the squares ranged from $25 \mathrm{~cm}^{2}$ when there was a single square to 
$2.77 \mathrm{~cm}^{2}$ when the set consisted of 9 squares. The arrangement of the squares was never the same for a specific number, this was true a) when the sets of squares were the target quantity, and b) when they were the response options. When the sets of squares were the response options, the colors were different from trial to trial. When target digit or sets of squares was presented alone (i.e., on tasks in which verbal number words were the response options) it was printed in a single rectangle located in the center of the card with the dimensions described above. When both the target and the comparison pair were visual stimuli, the target digit or set of squares was printed in black and enclosed within a single rectangle placed in the center of the card. Finally, when the target number or response options were verbal number words, these were repeated twice in order to reduce demands on the children's working memory.

Children received 1 point for every correct response on the matching tasks. For the logistic analyses, a child was considered to have formed the mapping between two number representations when he/she succeeded on 2 out of the 3 trials for each target number. The matching tasks ended when the child failed on three consecutive target numbers (i.e., failed on 2 out of 3 trials for three consecutive numbers). Children were always presented with the small set followed by the medium and finally the large set with the trials presented in a fixed order on all tasks; thus, although not all children completed the 27 trials, all children were presented with the complete small set size and were stopped when performance was chance level for nine trials. This lenient testing/scoring system was designed to provide our young participants with the opportunity to demonstrate their mapping ability while not being excessively demanding on children who repeatedly failed. 


\subsubsection{Quantity-to-number-word}

For this task, the stimuli binder contained 27 cards depicting a single rectangle with a set of colorful squares placed in the center. The experimenter told the child that they were going to play a game with Dolly and Belle; then she placed a puppet at either side of the stimuli binder and told the child that she would show him/her some squares, and then Dolly would say a number, and Belle would say another number, and that he/she would have to point to the puppet that said the number that told them how many squares there were on the card. The puppet that said the correct answer varied from trial to trial in a pseudo-randomized manner. The experimenter would specifically say: "I am going to show you some squares, you have to listen to Dolly and Belle and point to the puppet that says this (pointing to the set of squares) number of squares"

\subsubsection{Number-word-to-quantity}

The stimulus binder for this task contained 27 cards depicting two rectangles each with a different color set of squares that composed the response options. The experimenter told the child that one of the puppets would tell him/her the number of squares needed and that he/she needed to then point to the rectangle that contained the number of squares that the puppet had asked for. The side on which the correct answer was placed varied from trial to trial in a pseudo-randomized manner. The experimenter would specifically say: "Dolly needs you to find some squares for her. Listen to the number she will say and point to the side of this card that has that number of squares."

\subsubsection{Number-word-to-digit}

The stimulus binder for this task contained 27 cards with two rectangles each of which contained a digit (each one of a different color). The experimenter told the child that one 
of the puppets would say a number word and that he/she then had to point to the number that was the same as the number word the puppet had said. The side on which the correct answer was placed varied from trial to trial in a pseudo-randomized manner. The experimenter would specifically say: "Dolly needs you to find some numbers. Listen to the number she will say. Now point to the side of this card that has the number that Dolly said."

\subsubsection{Digit-to-number-word}

The stimulus binder for this task contained 27 cards with a single rectangle enclosing a colorful digit placed in the center. The experimenter told the child that she would show him/her a number and then Dolly would say a number word and Belle would say another number word and that he/she then had to point to the puppet that had said the number word that matched the number shown on the card. The side on which the correct answer was placed varied from trial to trial in a pseudo-randomized manner. The experimenter would specifically say: "I am going to show you a number, you have to listen to Dolly and Belle and point to the puppet that says this (pointing to the digit) number"

\subsubsection{Quantity-to-digit}

The stimuli binder for this task contained for each trial a card with the target stimuli (i.e., a set of black squares) which could held up by the experimenter, and a card which was laid flat on the binder and contained the response options which were two rectangles each of which enclosed a colorful digit. The experimenter told the child that he/she needed to look at the set of squares and then point to the rectangle that contained the digit that told them how many squares there were, so as to show the puppet. The side on which the correct answer was placed varied from trial to trial in a pseudo-randomized manner. The 
experimenter would specifically say: "I am going to show you some squares (pointing to the squares on the card), you have to point and show Dolly to the number that tells you how many squares there are here."

\subsubsection{Digit-to-quantity}

The stimuli binder for this task contained for each trial a card with the target stimulus (i.e., a black Arabic digit), this card could be held up by the experimenter for the child to see; and a card that remained flat on the binder that contained the response options which in this case consisted of two rectangles each of which enclosed a colorful set of squares. The experimenter told the child that he/she needed to look at the digit and then point to the side of the card that contained the number of squares that was the same as the digit in order to show the puppet the correct answer. The experimenter would specifically say: "I am going to show you a number (pointed to the card with the digit). Now point and show Dolly the number of squares that is the same as this (pointing to the digit) number."

\subsubsection{Comparison}

\subsubsection{Non-symbolic Comparison}

The purpose of this task was to assess children's ability to discriminate and compare two sets of objects and select the larger one (adapted from Chu, van Marle, \& Geary, 2013). Materials for this task included 16 small same colored foam cubes (approximately $3 \mathrm{~cm}$ high), and two paper gift bags (33 x $26 \times 13 \mathrm{~cm})$.

The experimenter placed the two gift bags in front of the child and said: "I am going to put some cubes into these bags. You need to watch very carefully and tell me which bag has more cubes.” The experimenter then proceeded to pick up the cubes, hold them in front of the child long enough to make sure the child saw each set and placed 
them inside the paper bags (while the child watched). Once the cubes were in the paper bags, the child could no longer see the cubes. On this task, children were not instructed to count and were not observed to explicitly attempt to count the cubes. On the warm-up trial, to ensure that the child understood the task requirements, the experimenter placed 1 cube inside one bag and 9 cubes in the other and asked the child: "Which bag has more cubes?" Feedback as to the correctness of the answer was only provided for this warm up trial. Subsequent trials included the following comparison sets presented in the following order: easy ratios $(1-2,3-6,4-7,2-3)$, followed by the difficult ratios $(3-4,6-8,4-5,7-9$, and 5-6). Children received 1 point for every correct response. The task ended when the child failed on 2 consecutive trials.

\subsubsection{Symbolic Comparison}

The verbal and written versions of the number comparison tasks included pairs of stimuli from three categories: comparisons with small numbers (i.e., 1-2, 2-3, and 3-4); mixed comparisons with one small number and one large number (i.e., 2-5, 3-6, and 4-7), and comparisons with large numbers (i.e., 5-7, 6-8, and 7-9). The items within each set were presented in randomized order but all children received the small set first, the mixed set second, and the large set last. Children received one point for every correct response. The task ended when the child failed on two consecutive trials. Both versions used a shopping context to introduce the comparisons (adapted from Purpura et al., 2013).

In the verbal number word version, the experimenter said to the child: "We are going to play a game pretending that we are going shopping for different fruits, OK?" The experimenter then presented the trials by saying: "We went to the store and I bought $n$ apples and you bought $n$ apples, who bought more apples?" 
For the written digit version, materials included nine white paper cards that contained two different color Arabic digits (1-9) printed within a rectangle. The experimenter said: "In this game we are going to pretend to go shopping for fruits. So, we went to the store and I bought this number of bananas (the experimenter pointed to one of the digits but did not say the number word) and you bought this number of bananas (the experimenter pointed to the other digit but did not say the number word), who has more?"

\subsection{Procedure}

Children were tested individually in a quiet area of the daycare $(n=66)$ or in their homes $(n=2)$ in two 25-min sessions no longer than one week apart. Depending on the age and ability of the child, the time taken to complete the tasks varied. Children were permitted to take short breaks as needed and were awarded colorful stickers after completing each task, however, if the child chose to stop testing, he or she was also awarded his/her sticker.

\section{Results}

Means, percent correct, and standard deviations for all of the tasks are shown in Table 3.1. Children could verbally count to 11, on average, although there was considerable variability. The range of counting was 0 to 100 . All the tasks had good reliabilities. Performance for each group of tasks was analyzed to explore effects of set size and is described below. Note that because the mapping tasks and the three comparison tasks ended after the child failed on three and two consecutive target numbers respectively, to avoid underestimating children's scores as a result of the trials that children did not see, these were scored as .5 to reflect chance performance. The resulting scores were used in all subsequent analyses. 


\subsection{Descriptive Analyses of Tasks}

\subsubsection{Knowledge of Representations}

\subsubsection{Quantity Knowledge}

Performance on the visible and hidden quantity reproduction tasks were analyzed in a 2 (task: visual, hidden) x 3 (set size: small, medium, and large) repeated measures ANOVA. As shown in Table 3.1, performance on the tasks decreased as the size of the set increased, $F(2,122)=266.28, M S E=0.59, p<.001, \eta_{p}{ }^{2}=.81$, and children performed significantly better when the set of cubes remained visually available than when they were hidden, $F(1,61)=28.20, M S E=0.47, p<.001, \eta_{p}{ }^{2}=.32$. The task by set size interaction was not significant $F(2,122)=2.91, M S E=0.37, p=.06, \eta_{p}{ }^{2}=.05$.

The number of cubes presented to the children on the small set size trials was within the subitizing range and thus their performance was quite good. However, for children to accurately solve the tasks when medium and large set sizes were involved, they needed to use an efficient counting strategy to determine the cardinal value of the set. Some children spontaneously counted the items in the visible quantity task according to observations by the experimenters. Partial correlations controlling for the effects of age revealed that children who scored higher on the verbal counting task also performed better on both the visible and hidden quantity reproduction tasks, $r(59)=.52, p<.001$, and $r(59)=.44, p<.001$, respectively. Differences between the visual and the hidden tasks presumably reflected children's limited ability to determine the cardinality of a set through counting and the stronger demands placed on children's working memory when the set of cubes was hidden throughout the trials. Nevertheless, their good performance on the small set size suggests they understood the demands of the quantity-matching task, 
even when the target set was hidden.

\subsubsection{Symbol Knowledge}

Performance on the symbolic representation tasks was analyzed in a 3 (task:

Number words vs. Words; Digits vs. Letters; and Digits vs. Quantities) x 3 (size: small, medium, large) repeated measures ANOVA. There were no significant effects. To test whether children learn to categorize number words before they learn to categorize digits, we determined the number of children who performed significantly above chance level on each task (i.e., obtained a score of 8 or $9, p<.05$ binomial probability). Twenty-four children (39\%) performed significantly above chance on the Number Word vs. Word task whereas only 16 children (26\%) performed above chance on the Digit vs. Letter task. The $13 \%$ difference in success across tasks was significant (95\% CIs [0.19\%, 25.07\%]), supporting the hypothesis that children recognize number words as a category of symbols prior to learning to distinguish Arabic digits (Bialystok, 1992).

The digits versus quantity task was excluded from subsequent analyses because the pattern of performance did not correspond to that of other tasks: Of the 16 children who performed above chance on the digit versus letter task (i.e., they were able to discriminate at least 8 of the 9 digits from letters), 31\% failed to consistently choose the digit as the way in which the puppet should write the number to denote a quantity (i.e., their performance was at chance) thus, to the children, both representations may have been equally valid ways to indicate quantity. It was not possible to tell from this task whether the children chose the analog representation because they were unaware that written symbolic representations are an efficient way to denote quantity or if children associated the fact that the experimenter said that there were physical objects (i.e., toys) inside the 
box and thus may have considered that the analog representations were more appropriate to represent those objects per se.

\subsubsection{Summary}

Children's performance on the knowledge of representation tasks indicates that many had a moderate level of knowledge about the component skills that are related to mapping and comparison tasks. However, some children had minimal knowledge of number words, and some were not able to distinguish number words or digits from other symbols. Verbal counting, number word versus word knowledge, and digits versus letter knowledge were used as predictors of mapping in subsequent analyses.

\subsubsection{Mapping Tasks}

To investigate the effect of set size and whether the direction of mapping influenced children's performance, performance on each pair of tasks was analyzed in a separate 2 (mapping direction) x 3 (set size: small, medium, large) repeated measures ANOVA. Means are shown in Table 3.1 and Figure 3.4; statistics for the analyses are shown in Table 3.3. Direction of mapping did not influence performance on the symbolic tasks (number-word-to-digit and digit-to-number-word): Children performed similarly whether they were mapping words to digits or digits to words. For the tasks that involved mapping between number words and quantities, there was a significant main effect of direction of mapping. Children performed better when mapping from a number word to the corresponding quantity than when mapping from a quantity to the number word (see also Odic et al., 2015). For the analyses involving the mappings between number words and digits and number words and quantities, the main effect of set/number size was significant. Children's scores decreased as the number/set sizes increased. Note, however, 
that the set size effect was much smaller for the symbol-symbol mapping task than for the word-quantity mapping tasks.

For the tasks involving quantities and Arabic digits, as with the other mapping tasks, there was a significant main effect of size, there was no main effect of direction of mapping, and the direction by size interaction was significant. The pattern of results show that at the small set size, children performed significantly better when mapping from digits to quantities $(M=6.19)$ than when mapping from quantities to digits $(M=5.62)$, $F(1,61)=6.05, M S E=1.68, p=.017, \eta_{p}{ }^{2}=.09$. At the medium set size, there was no difference in performance between the two tasks (digit-quantity $M=4.80$; quantity-digit $M=4.86), F(1,61)=0.08, M S E=1.69, p=.78, \eta_{p}^{2}=.00$. For the large set size however, the children performed better when mapping from quantities to digits $(M=4.59)$ than when mapping from digits to quantities task $(M=4.17), F(1,61)=4.78, M S E=1.14, p=$ $.03, \eta_{p}{ }^{2}=.07$. 


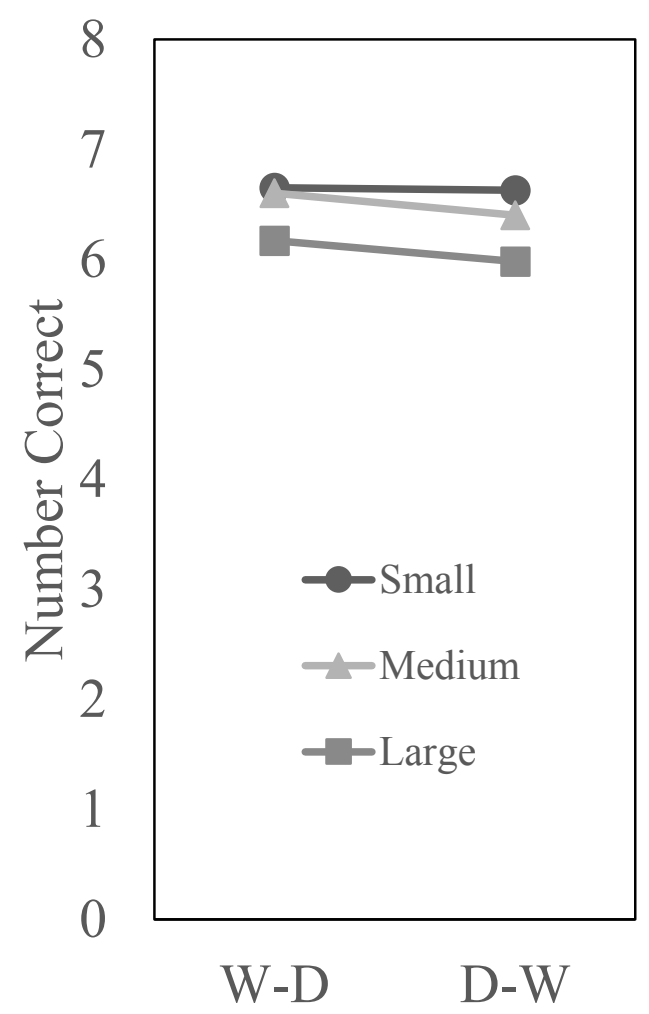

Direction
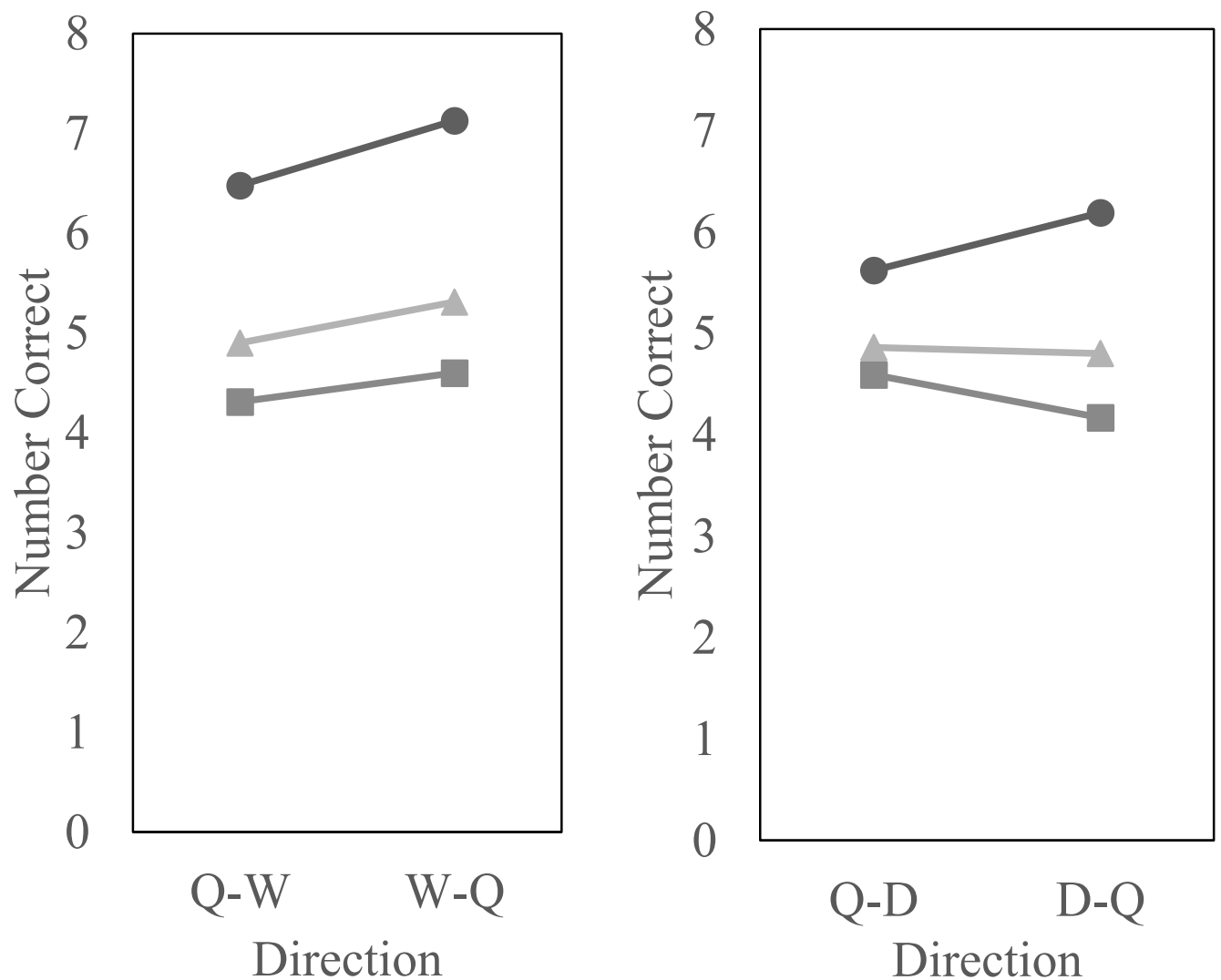

Figure 3.4. Number correct for each pair of mapping tasks. Direction of mapping by set size (small, medium, large). 
Table 3.3. Inferential statistics for analyses of the pairs of mapping tasks.

\begin{tabular}{|c|c|c|c|c|c|c|c|c|c|c|}
\hline \multirow[b]{2}{*}{ Variable } & \multirow[b]{2}{*}{$d f$} & \multicolumn{3}{|c|}{ Word-Digit } & \multicolumn{3}{|c|}{ Quantity-Word } & \multicolumn{3}{|c|}{ Quantity-Digit } \\
\hline & & $F$ & $M S E$ & $\eta_{p}^{2}$ & $F$ & $M S E$ & $\eta_{p}^{2}$ & $F$ & $M S E$ & $\eta_{p}^{2}$ \\
\hline Direction & 1,61 & 0.72 & 2.44 & .01 & $9.45 * *$ & 2.01 & .13 & 0.05 & 1.59 & .00 \\
\hline Size $^{1}$ & 2,122 & $3.78 *$ & 2.86 & .06 & $38.27 * * *$ & 4.79 & .39 & $15.75 * * *$ & 5.33 & .20 \\
\hline Direction by Size & 2,122 & 0.22 & 1.58 & .00 & 0.61 & 1.64 & .01 & $5.27 * *$ & 1.52 & .08 \\
\hline
\end{tabular}

$* p<.05, * * p<.01, * * * p<.001$

${ }^{1}$ Greenhxouse-Geisser adjusted degrees of freedom were $F(1.82,111.38)$ for size effect for Quantity-Digit analysis. 


\subsubsection{Performance on Comparison Tasks}

Children's performance on the non-symbolic comparison task was analyzed in a 2(ratio: smaller [.50 to .66], larger [.75 to .83]) ANOVA: they performed better on trials with large ratios $(M=2.88, S D=1.08)$, than on small ratios $(M=2.56, S D=1.09), F(1$, $61)=5.08, M S E=0.64, p=.028, \eta_{p}{ }^{2}=.08$.

Performance on the symbolic comparison tasks was analyzed in a 2(task: verbal and digit comparison) x 3(comparison number sizes: small, medium and large) repeated measures ANOVA. Means and standard deviations are presented in Table 3.1. There were no significant effects. The lack of significant effects could reflect the overall difficulty children had when performing symbolic number comparisons.

\subsection{Relations among precursor, mapping, and comparison skills}

Path analysis was used to determine the relations among the precursor, mapping, and comparison skills, testing the hypotheses that (a) knowledge about the individual representations was needed before children could succeed at the mapping tasks, and (b) mapping between the symbol and quantity representations was needed before children could succeed at the symbolic comparison tasks. Before conducting the path analyses we created composite variables ${ }^{5}$ to index a) quantity knowledge (i.e., children's ability to reproduce quantities from the average of the visible quantity reproduction and the hidden quantity reproduction tasks); b) symbol knowledge (i.e., children's ability to identify the number symbols as individual representations; this variable was created from the average of the scores on the number-word versus word task and digit versus letter tasks); and c) mapping skills: each of the three mappings between representations (i.e., number word-

\footnotetext{
${ }^{5}$ Latent variables were not used because we had only two indicators for each construct.
} 
quantity, number word-digit, and digit-quantity paths) by averaging across the two directions (i.e., quantity-to-number-word and number-word-to-quantity, number word-todigit and digit-to-number-word, quantity-to-digit and digit-to-quantity). Correlations among these composite variables, age, verbal counting, and the three comparison tasks, as well as means and standard deviations of the composite variables are presented in Table 3.4.

As shown in Table 3.4, except for verbal counting and verbal comparison, children's performance was correlated with age. After partialling out age, children's knowledge of verbal counting was correlated with their ability to identify number symbols and their ability to reproduce quantities. Consistent with the view that quantitative and symbolic knowledge initially are separable (e.g., LeFevre et al., 2010), children's ability to identify number symbols was unrelated to their ability to reproduce quantities. Children's verbal counting, their ability to identify the number symbols and their ability to reproduce a set of objects were all significantly correlated with children's mapping abilities. The mapping variables were highly intercorrelated. Children's performance on the comparison tasks was significantly correlated with both symbol-toquantity mapping tasks. The model shown in Figure 3.2 was tested with M Plus 7.31 (Muthén \& Muthén, 1998-2015). Results from the analysis are summarized in Tables 3.5 and 3.6, and shown graphically in Figure 3.5. 
Table 3.4. Zero order correlations (below the diagonal) among age, verbal counting, all composite variables, and comparison tasks. Partial correlations controlling for age above the diagonal.

\begin{tabular}{|c|c|c|c|c|c|c|c|c|c|c|}
\hline & & \multicolumn{3}{|c|}{ Precursor Skills } & \multicolumn{3}{|c|}{ Mapping Tasks } & \multicolumn{3}{|c|}{ Comparison Tasks } \\
\hline & Age & $\begin{array}{l}\text { Verbal } \\
\text { count }\end{array}$ & $\begin{array}{c}\text { Symbol } \\
\text { Knowledge }\end{array}$ & $\begin{array}{l}\text { Quantity } \\
\text { knowledge }\end{array}$ & $\begin{array}{l}\text { Word- } \\
\text { Digit }\end{array}$ & $\begin{array}{l}\text { Word- } \\
\text { quantity }\end{array}$ & $\begin{array}{l}\text { Digit- } \\
\text { quantity }\end{array}$ & Quantity & Word & Digit \\
\hline Verbal Counting & .21 & & $.49 * * *$ & $.55 * * *$ & $.40 * * *$ & $.55 * * *$ & $.42 * * *$ & $.37 * *$ & $.40 * * *$ & .11 \\
\hline Symbol knowledge & $.33 * *$ & $.52 * * *$ & & .22 & $.63 * * *$ & $.36^{* *}$ & $.48 * * *$ & .18 & .18 & .17 \\
\hline Quantity & $.33 * *$ & $.58 * * *$ & $.31 *$ & & $.42 * * *$ & $.57 * * *$ & $.58 * * *$ & .18 & $.46 * * *$ & .19 \\
\hline \multicolumn{11}{|l|}{ knowledge } \\
\hline \multicolumn{11}{|l|}{ Mapping } \\
\hline Word-digit & $.42 * * *$ & $.45 * * *$ & $.68^{* * *}$ & $.50 * * *$ & & $.53 * * *$ & $.65^{* * *}$ & .25 & .23 & .19 \\
\hline Word-quantity & $.41 * * *$ & $.58 * * *$ & $.44 * * *$ & $.63 * * *$ & $.61 * * *$ & & $.75^{* * *}$ & $.30^{*}$ & $.46 * * *$ & $.26^{*}$ \\
\hline Digit-quantity & $.34 * * *$ & $.45 * * *$ & $.54 * * *$ & $.63 * * *$ & $.70 * * *$ & $.78 * * *$ & & $.35 * *$ & $.46^{* * *}$ & $.38 * * *$ \\
\hline \multicolumn{11}{|l|}{ Comparison } \\
\hline Quantity & $.32 *$ & $.41 * * *$ & $.27 *$ & $.27 *$ & $.35^{* *}$ & $.39 * *$ & $.42 * * *$ & & $.38 * *$ & .11 \\
\hline Word & .17 & $.42 * * *$ & .23 & $.49 * * *$ & $.27 *$ & $.48 * * *$ & $.49 * * *$ & $.41 * *$ & & $.46 * * *$ \\
\hline Digit & $.25^{*}$ & .15 & .23 & $.26^{*}$ & $.27^{*}$ & $.33 * *$ & $.43 * *$ & .18 & $.48 * * *$ & \\
\hline Means & & & & & 19.21 & 16.36 & 15.12 & & & \\
\hline$S D$ & & & & & 6.06 & 3.76 & 3.77 & & & \\
\hline
\end{tabular}

Note. ${ }^{*} p<.05 ; * * p<.01, * * * p<.001$ 
Table 3.5. Path analysis: Standardized direct, indirect and total effects.

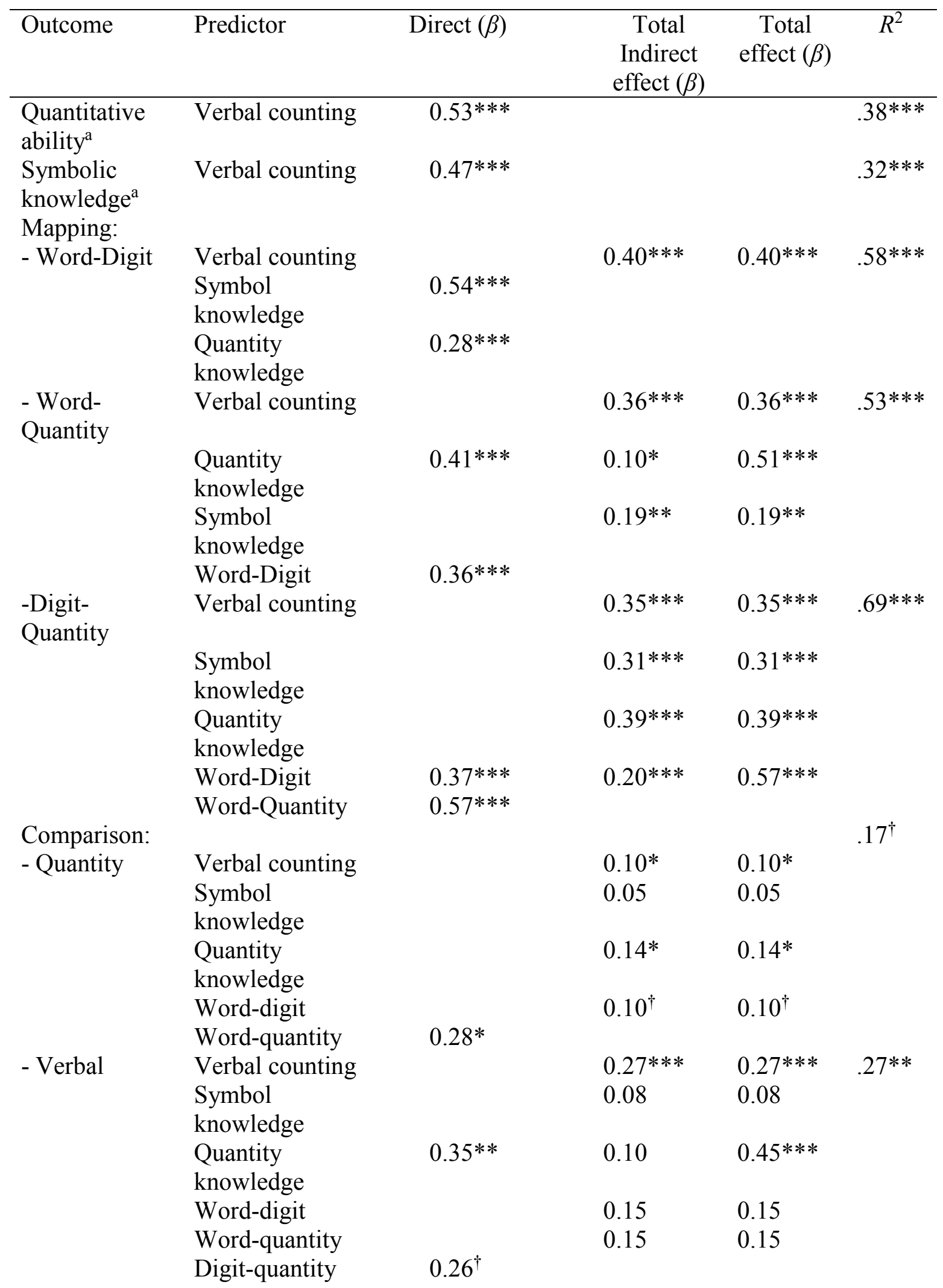




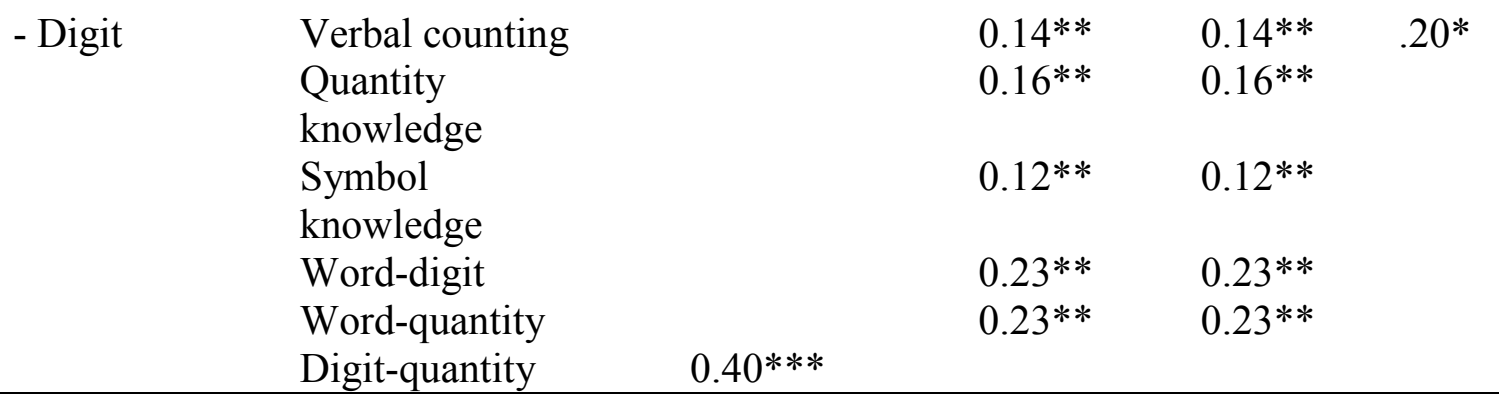

Note. The $R^{2}$ for each outcome includes all the listed variables as predictors. ${ }^{\dagger} p<.062,{ }^{*} p<.05, * * p<.01, * * * p<.001,{ }^{a}$ age was a significant predictor. 


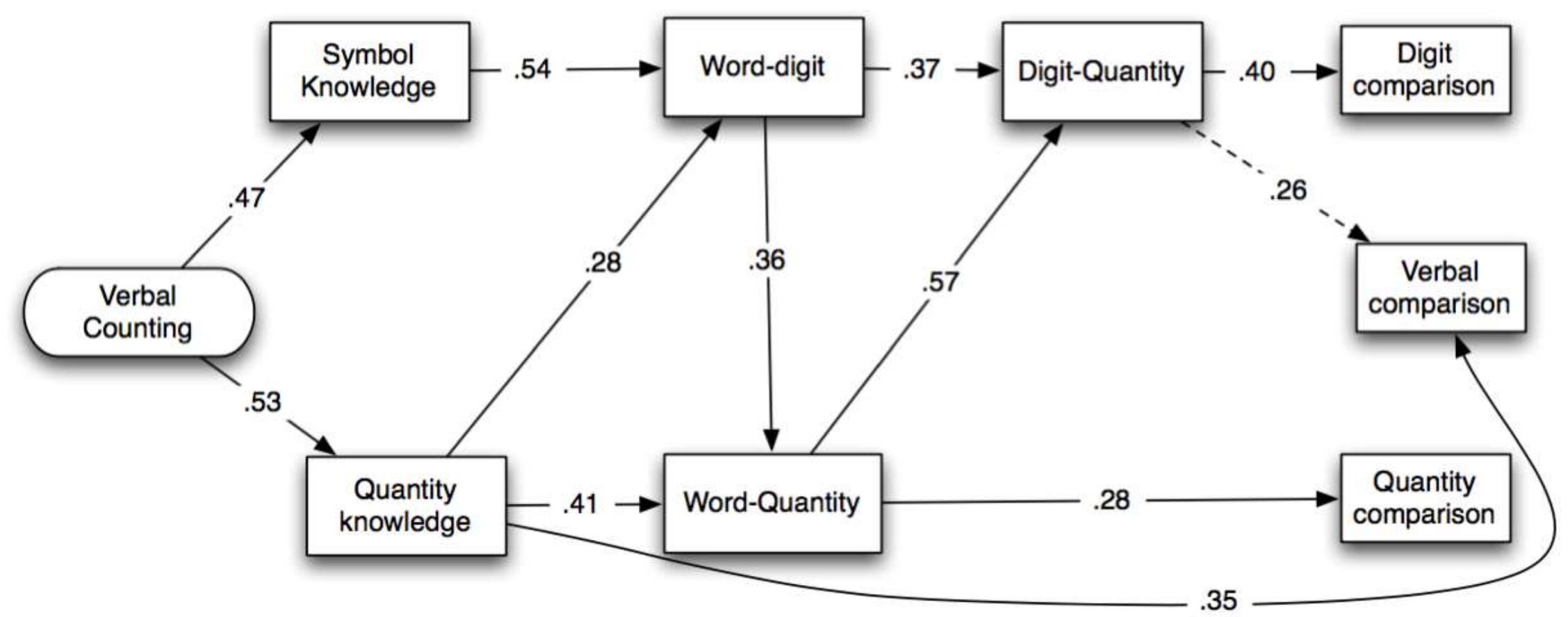

Figure 3.5. Path model of the relations among children's precursor skills, mapping performance, and comparison tasks. All significant (standardized) direct effects are shown. Dotted line $p=.061$. 
Table 3.6. Path analysis: Other significant (standardized) indirect effects.

\begin{tabular}{|c|c|c|}
\hline Outcome & Indirect Effects & Estimate \\
\hline \multirow{2}{*}{ Word-Digit } & Verbal count through symbol knowledge & $0.25 * * *$ \\
\hline & Verbal count through quantity knowledge & $0.15^{* *}$ \\
\hline \multirow{5}{*}{ Word-quantity } & Verbal count through quantity knowledge & $0.22 * * *$ \\
\hline & $\begin{array}{l}\text { Verbal count through symbol knowledge and word- } \\
\text { digit }\end{array}$ & $0.09 *$ \\
\hline & $\begin{array}{l}\text { Verbal count through quantity knowledge and word- } \\
\text { digit }\end{array}$ & $0.05^{*}$ \\
\hline & Symbol knowledge through word-digit & $0.19 * *$ \\
\hline & Quantity knowledge through word-digit & $0.10^{*}$ \\
\hline \multirow[t]{11}{*}{ Digit-quantity } & $\begin{array}{l}\text { Verbal count through quantity knowledge and word- } \\
\text { quantity }\end{array}$ & $0.12 * *$ \\
\hline & Verbal count through symbol knowledge, word-digit & $0.09 * *$ \\
\hline & $\begin{array}{l}\text { Verbal count through quantity knowledge, word- } \\
\text { digit }\end{array}$ & $0.05^{*}$ \\
\hline & $\begin{array}{l}\text { Verbal count through symbol knowledge, word-digit, } \\
\text { and word-quantity }\end{array}$ & $0.05^{*}$ \\
\hline & $\begin{array}{l}\text { Verbal count through quantity knowledge, word } \\
\text { digit, and word quantity }\end{array}$ & $0.03 *$ \\
\hline & Symbol knowledge through word-digit & $0.20 * * *$ \\
\hline & $\begin{array}{l}\text { Symbol knowledge through word-digit and word- } \\
\text { quantity }\end{array}$ & $0.11 * *$ \\
\hline & Quantity knowledge through word-quantity & $0.23 * * *$ \\
\hline & Quantity knowledge through word-digit & $0.10^{*}$ \\
\hline & $\begin{array}{l}\text { Quantity knowledge through word-digit, and word } \\
\text { quantity }\end{array}$ & $0.06^{*}$ \\
\hline & Word-digit through word-quantity & $0.20 * * *$ \\
\hline \multirow{2}{*}{$\begin{array}{l}\text { Quantity } \\
\text { comparison }\end{array}$} & Quantity knowledge through word-quantity & $0.11 *$ \\
\hline & Word-digit through word-quantity & $0.10^{\dagger}$ \\
\hline $\begin{array}{l}\text { Verbal } \\
\text { comparison }\end{array}$ & Verbal counting through quantity knowledge & $0.18^{*}$ \\
\hline \multirow[t]{6}{*}{$\begin{array}{l}\text { Digit } \\
\text { comparison }\end{array}$} & $\begin{array}{l}\text { Verbal count through quantity knowledge, word- } \\
\text { quantity, and digit-quantity }\end{array}$ & $0.05^{*}$ \\
\hline & $\begin{array}{l}\text { Verbal count through symbol knowledge, word-digit, } \\
\text { and digit-quantity }\end{array}$ & $0.04 *$ \\
\hline & $\begin{array}{l}\text { Verbal count through quantity knowledge, word- } \\
\text { digit, and digit-quantity }\end{array}$ & $0.02^{\dagger}$ \\
\hline & $\begin{array}{l}\text { Verbal count through symbol knowledge, word-digit, } \\
\text { word-quantity, and digit-quantity }\end{array}$ & $0.02^{\dagger}$ \\
\hline & $\begin{array}{l}\text { Symbol knowledge through word-digit and digit- } \\
\text { quantity }\end{array}$ & $0.08^{*}$ \\
\hline & Symbol knowledge through word-digit, word- & $0.04 *$ \\
\hline
\end{tabular}


quantity, and digit-quantity

Quantity knowledge through word-quantity and $\quad 0.09^{*}$ digit-quantity

Quantity knowledge through word-digit, and digit- $\quad 0.04^{*}$ quantity

Word-digit through digit-quantity $0.15^{* *}$ Word-digit through word-quantity, and digit-quantity $\quad 0.08^{*}$ Word-quantity through digit-quantity $0.23^{* *}$

Note. Estimate is the standardized coefficient. ${ }^{\dagger} p<.062,{ }^{*} p<.05,{ }^{* *} p<.01,{ }^{* * *} p<.001$.

The initial model showed a poor fit to the data $\chi^{2}(23)=44.73 p=.004$, RMSEA $=$ $.12,90 \% \mathrm{CI}[.068-.177] \mathrm{CFI}=.92, S R M R=.09$. Based on the modification indices, paths from quantity knowledge to a) word-digit mapping and to b) verbal comparison were added to the model; the addition of these paths significantly improved the model fit, $\chi^{2}$ $(21)=28.43, p=.13, R M S E A=.076,90 \%$ CI $[0.00-0.14], C F I=.97, S R M R=.052$ and this model was retained.

As shown in Figure 3.5, the final model was generally consistent with our hypothesized model. Children's verbal counting ability predicted their knowledge of the number symbols and their ability to understand and manipulate small exact quantities. Quantity and symbol knowledge were found to be separate precursor skills which, as expected, had direct effects on the mappings between number words and digits and number words and quantities. Specifically, both symbol and quantity knowledge predicted children's knowledge of the mapping between number words and digits. The path from quantity knowledge to word-digit mapping was not hypothesized, however, in order to be successful on the quantity knowledge tasks, children need to have a greater understanding of the symbolic nature of the number words in order to use them to accurately determine the quantity of a set. It is possible that a better understanding of the number words as symbols facilitates children's ability to associate them to the written 
symbolic counterparts. Quantity knowledge was directly and indirectly related to children's word-quantity mapping through their ability to associate number words to the corresponding digits. Word-quantity mapping also had a significant indirect effect from symbol knowledge through word-digit mapping.

We found evidence in support of the hypothesized pattern of relationships among the mapping skills: word-digit mapping predicted word-quantity mapping and both of these abilities predicted the mapping between digits and quantities. As hypothesized, children's ability to compare two exact non-symbolic quantities was directly related to their word-quantity mapping. Verbal comparison was marginally related to children's digit quantity mapping and significantly predicted by children's quantity knowledge suggesting that being able to compare two verbal number words requires an understanding of the association between quantities and number symbols. Children's ability to compare two written numbers was directly related to the mapping between digits and quantities as expected. The present data further suggest that the ability to compare two symbolic number representations is built upon the development and integration of the hypothesized precursor and mapping skills as shown by the multiple significant indirect effects from the all precursor and mapping skills to digit comparison (see Table 3.6).

\subsection{Order of acquisition of the mappings}

To test the order in which children acquire the mappings among digits, words, and quantities, we examined the relations among the mapping tasks with logistic regression analyses. Children's performance on the mapping tasks was categorized as either at or above chance (i.e., obtained a score of 19 or higher on the 27 trials, $p<.05$ ). Children's 
level of performance on both tasks for each mapping pathway (see Figure 3.1) was used to classify them into combined categories of those children who showed above chance performance on both tasks and those who performed at chance on one or both tasks. The percentage of children in each category for each of the six mapping tasks and for the combined task categories are shown in Table 3.7. The pathway numbers refer to those presented in Figure 3.1 .

Table 3.7. The percentage of children who performed above chance on each of the three sets of mapping tasks.

The combined column indicates the percentage of children who performed above chance on both orders for that pair of tasks.

\begin{tabular}{llccc}
\hline & & \multicolumn{2}{c}{ Direction } & Combined \\
\cline { 3 - 4 } Pathway & Mapping Tasks & 1 & 2 & \\
\hline 1 & Number Words, Digits & 53 & 50 & 42 \\
2 & Quantities, Number words & 26 & 37 & 21 \\
3 & Quantities, Digits & 23 & 21 & 15 \\
\hline
\end{tabular}

Note. Direction 1 corresponds to the order of the tasks in the Mapping Tasks column.

As shown in Table 3.7, more children successfully mapped between number words and digits than between quantities and number words. Children were least likely to be successful mapping between quantities and digits. Results from the logistic regression analyses are summarized in Table 3.8. Children who failed to map between number words and digits had only a 3\% probability of successfully mapping digits to quantities; however, if they successfully mapped number words and digits, the probability that they successfully mapped digits and quantities was 31\%. Children who failed on mapping 
quantities and number words had only a $2 \%$ probability of successfully mapping digits and quantities, whereas children who successfully mapped quantities and number words had a $62 \%$ probability of success on digit-quantity mapping. As shown in Table 3.8 , the odds ratio for predicting digit-quantity mapping was larger for number word-quantity mapping as compared to the odds ratio resulting when number word-digit mapping was used as the predictor. This is consistent with Bialystok's (1992) claims about the importance of cardinality knowledge for mapping written digits to quantities. In summary, both digit-word and word-quantity mapping predicted the probability that children would perform above chance on the digit-quantity mapping path ${ }^{6}$.

Table 3.8. Results from logistic regression analyses (numbers in parentheses refer to the pathways shown in Figure 3.1).

\begin{tabular}{|c|c|c|c|c|c|}
\hline \multirow{2}{*}{$\begin{array}{c}\text { Dependent } \\
\text { Variable }\end{array}$} & \multirow[b]{2}{*}{ Predictor } & \multirow[b]{2}{*}{$\chi^{2}(1, N=62)$} & \multirow[t]{2}{*}{$\begin{array}{c}\text { Odds } \\
\text { Ratio }^{a}\end{array}$} & \multicolumn{2}{|c|}{$\begin{array}{l}95 \% C I \text { for } \\
\text { Odds Ratio }\end{array}$} \\
\hline & & & & $L L$ & $U L$ \\
\hline $\begin{array}{l}\text { Digit-quantity } \\
\text { (3) }\end{array}$ & Number word-digit (1) & $10.13 * *$ & 15.56 & 1.80 & 134.23 \\
\hline $\begin{array}{l}\text { Digit-quantity } \\
\text { (3) }\end{array}$ & $\begin{array}{l}\text { Number word-quantity } \\
\text { (2) }\end{array}$ & $24.27 * * *$ & 76.80 & 7.91 & 746.18 \\
\hline
\end{tabular}

Note: Mapping variables used are the combined tasks.

$* p<.05, * * p<.01, * * * p<.001$.

${ }^{a}$ Odds of success on dependent variable given success on the predictor.

\section{Discussion}

The goals of the present study were to (a) explore the relations among children's knowledge of the mappings among quantities, number words, and digits and (b) assess a model of how the mapping tasks fit into a framework for early numeracy acquisition. We

\footnotetext{
${ }^{6}$ We hypothesized that word-digit mapping would develop prior to word-quantity mapping; in support of this hypothesis, the contingency table presented in Appendix C shows that none of the children who performed at chance on word-digit mapping were successful on word-quantity mapping. Logistic regression analyses were not performed given the observed complete separation.
} 
found that children's performance on number word-digit mappings was better, across set sizes, than on the other two mapping pathways. Digit-quantity mappings were most difficult, as expected. Children were more likely to show evidence for digit-quantity mapping if they had better performance on the other two pathways. Mapping abilities were predicted by children's knowledge about symbols and quantities, and digit-quantity mapping predicted children's performance on the digit comparison task and was marginally related to the verbal number comparison task. Thus, the current research suggests that digit-quantity mapping is a central feature of children's early numeracy acquisition, that it develops after the other two mapping pathways, and that it represents the integration of knowledge about symbols and quantities.

\subsection{Precursors of mapping skill}

As hypothesized, both quantity and symbol knowledge were related to performance on mapping tasks. These two kinds of knowledge formed different pathways consistent with the linguistic/symbolic and quantitative pathways described in LeFevre et al. (2010; Sowinski et al., 2015). Both quantity and symbol knowledge predicted performance on number word-digit mapping. In contrast, performance on number wordquantity mapping was predicted by quantity knowledge, and indirectly by symbol knowledge.

Children's verbal counting was directly related to both symbolic and nonsymbolic precursor skills and had indirect effects on all mapping and symbolic number comparison tasks. Verbal counting is presumably related to both symbolic/linguistic and quantity pathways because it requires production of symbols (words) and learning the ordinal rule that links number words to relative quantities. Ordinal knowledge measured 
in older children and adults is a significant predictor of arithmetic skill (Lyons \& Beilock, 2011; Lyons et al., 2014). Verbal counting presumably captures the earliest stages of ordinal knowledge. Counting higher than 10 also may indicate children's early understanding of the structure of the number system. Accordingly, in the present research, verbal counting formed an important precursor to mapping performance.

\subsection{Order in which children learn mappings across representations}

The present research supported Bialystok's (1992) view that children acquire the mappings between quantities and digits after they have learned to map between digits and number words and between number words and quantities (see also Batchelor et al., 2015; Knudsen et al., 2015; von Aster \& Shalev, 2007). In addition, although some children who successfully mapped digits to number words failed to map number words to quantities, none of the children who failed mapping number words to digits successfully mapped number words to quantities (Appendix C). Together, these results provided evidence that children learn the mapping between number words and digits, then between number words and quantities, and finally between digits and quantities (Bialystok, 1992). In contrast, Benoit et al. (2013) concluded that children develop the links between number words and quantities and quantities and digits before they map digits to number words. In the present study, results from the logistic regression analyses showed that children who failed to map number words to digits and number words to quantities failed on digit-quantity mapping. These results support the hypothesis that children need to develop the links between digits and number words and between number words and quantities before they can associate each digit to the corresponding quantity (Bialystok, 1992). 
One possible source of the large discrepancy between the present results and those of Benoit et al. is that, in their study, task requirements were not equivalent for the various mapping tasks. That is, on the two tasks on which children were asked to map from quantities or digits to spoken number words, they were required to produce the number word after being shown the quantity or digit. In contrast, for the four other mappings, the target quantity or digit was selected from the set of six targets, and thus recognition was required instead of recall. In the present study, all mapping tasks were presented such that children chose the target from two alternatives, thus equating the task difficulty across all six tasks. A second source of discrepancy could have been that the quantities (arrays of dots) used in Benoit et al.'s study were arranged in the familiar patterns that are used on dice. If children recognized these specific patterns, the mappings with quantities may have been easier than if the children had to count to determine the quantity and match it to the number word or digit (Mandler \& Shebo, 1982). A third source of discrepancy could be that the present study used the numbers 1-9 whereas Benoit et al., used numbers 1-6. In order to succeed on the symbol-quantity mapping tasks with larger numbers, children needed to use an efficient counting strategy. Thus, it is possible that the lower performance displayed by the children on the symbol-quantity tasks is due to their underdeveloped ability to determine the cardinality of larger sets; in contrast, children could have shown better performance when mapping number words to digits because no counting was required for those tasks.

Finally, children have different educational experiences that might influence the order in which they learn the mappings. The children in Benoit et al.'s research were French speaking, older, and recruited from schools, whereas the children in the present 
research were English speaking, younger, and recruited from daycares. More research is needed to determine whether the order which children learn the mappings across number representations varies depending on language, instruction, or other demographic or educational factors.

\subsection{Predictors of Magnitude Comparisons}

Results from the path analysis showed that performance on the digit comparison task was predicted directly by children's digit-quantity mapping, with a significant indirect effect of the word-digit and word-quantity mappings. Thus, children's symbolic comparison skill was related to their ability to integrate knowledge of the verbal and written number symbols as well as to connect them to the corresponding quantity. These results are consistent with Bialystok's (1992) proposal that children are only able to use number symbols to solve mathematical tasks (indexed here by the symbolic comparison tasks) once they have mastered the links among the three representations of number. In previous studies, researchers have examined the relations between children's mathematical skills and quantity-number word mapping in subitizing and counting tasks (e.g., Landerl, 2013; LeFevre et al., 2010; Titeca, Roeyers, Josephy, Ceulmans, DeSoete, 2014) or digit-number mapping in digit naming tasks (e.g., Kolkman et al, 2013;

Krajewski \& Schneider, 2009; LeFevre et al., 2010). However, the present study provides a more complete picture of the role of children's mapping abilities because it also included digit-quantity mapping. Digit-quantity mapping appears to be a crucial skill that children must master before they can move on to more complex mathematical tasks (Knudsen et al., 2015; Purpura et al., 2013).

\subsection{Model of Early Numeracy Acquisition}


As outlined in Figures 3.2 and 3.5, the results of the present research were broadly consistent with a framework for early number development that integrated precursor, mapping, and comparison skills and was informed by a description of the skill acquisition process outlined by von Aster and Shalev (2007). In the integrated model, the various precursor and mapping tasks were arranged into a linguistic/symbolic and a quantitative pathway, consistent with LeFevre et al. (2010). As shown in Figure 3.5, children's ability to discriminate number symbols and their ability to manipulate and understand quantities initially formed separate precursor paths. Symbol knowledge predicted word-digit mapping and quantity knowledge predicted word-quantity mapping. However, quantity knowledge also had a direct relation to word-digit mapping and symbol knowledge was related to children's word-quantity mapping through their ability to link number words to the corresponding digit (see Table 3.6). These results suggest that, in learning to map number words to digits and to quantities, children draw upon and integrate the initially separate precursor knowledge of both the number symbols and of quantities.

Children's digit-quantity mapping was, as expected, predicted by the mappings between number words and digits and number words and quantities. The development of the digit-quantity mapping is particularly relevant because it directly predicted written symbolic number comparison. Thus, the present results suggest that the development of children's more advanced numeracy skills is a process in which separate symbolic and quantitative skills merge to allow children to further their mathematical knowledge.

Figure 3.5 shows that word-quantity mapping was indirectly related to digit comparison task performance through the digit-quantity mapping (see Table 3.6). Thus, consistent with other theorists (von Aster \& Shalev, 2007) the present results provide 
evidence for the central role that children's cardinality knowledge plays in facilitating the acquisition of more advanced numeracy skills (cf. Batchelor et al., 2015). Further research is needed, however, to explore ways to enhance the integration of cardinality and word-digit knowledge in the preschool years.

\subsection{Limitations of the Present Research}

Some limitations of the present study should be considered. First, our sample size was relatively small which means that our model will need to be replicated in a larger sample in order to ensure generalizability of the results. Although we started with a theoretically motived model, which somewhat mitigates this issue, correlational methods like path analysis can be misleading in moderately-sized samples. The advantage of considering all the variables simultaneously, rather than conducting a large number of separate regressions, was also a factor in our decision to use this statistical approach. Second, although prior research has found that children are able to link digits to number words before they associate number words to quantities (e.g., Mix, 2009) and that the mapping between digits and quantities is the last to develop (e.g., Knudsen et al., 2015), the present cross-sectional data do not enable us to determine whether the obtained pattern of results on the mapping tasks reflects the developmental progression. Thus, future replication of the proposed model in a longitudinal study is desirable. A final consideration in interpreting these results was that, although we found the expected relations between children's digit-quantity mapping and symbolic comparisons (especially digit comparison), children's performance on the symbolic comparison tasks was quite poor, presumably because they were young and in the process of creating the mappings among the representations. Replication with longitudinal samples in which the 
development of the mapping and comparison skills was tracked over time are necessary to understand these relations more fully.

\subsection{Conclusions}

The results of this study showed that young children's knowledge of the symbols used to denote number predicted mapping between symbolic and non-symbolic representations of quantities, and that knowledge of mapping digits to quantities predicted their verbal and digit magnitude comparisons. These findings support the view that integrated knowledge of symbolic and non-symbolic representations of quantity contribute to children's success on magnitude comparisons tasks. Strong links between symbolic skills and early numeracy have been observed in many studies (e.g., LeFevre et al., 2010; Lyons et al., 2014; Purpura, Hume, Sims, \& Lonigan, 2011; Purpura et al., 2013). Symbolic number comparison tasks predict children's arithmetic skills from grade 1 on (Holloway \& Ansari, 2008, 2009; Lyons et al., 2014; Soltész et al., 2010), and automatic access to semantic (i.e., quantity) knowledge through digits is characteristic of adults' performance (Castronovo \& Göbel, 2012). Further, children with math difficulties appear to struggle with mapping between quantities and symbols (Noël \& Rousselle, 2011; Rousselle \& Noël, 2007). The present findings corroborate those results and suggest that interventions that help children integrate number word and quantity knowledge with digits may be important for those individuals with numerical difficulties. In the next chapter I test the model proposed in the present study in a sample of Canadian and Mexican preschool children. 


\section{CHAPTER 4: STUDY 2}

The results of Study 1 showed that verbal counting, and their ability to represent and manipulate quantities ${ }^{7}$ are significant predictors of children's mapping skills. Furthermore, children's mapping abilities were related to performance on symbolic number comparison tasks. In addition, children in Study 1 performed better when mapping number words to digits and their performance was worse when mapping digits to quantities. Furthermore, none of the children who performed at chance on the worddigit mapping were successful at mapping number words to quantities. Taken together, these findings provided evidence that the first mapping to develop is that between number words and digits, children then develop that between number words and quantities, and that the mapping between digits and quantities developed last. In contrast, Benoit et al. (2013) found that the mapping between number words and digits developed after the symbol-quantity mappings. They suggested that educational experiences could have contributed to the encountered pattern of results.

The main goal of Study 2 was to assess whether the relationships among early numeracy skills would show a similar pattern in children with different cultural and educational backgrounds. Canadian and Mexican three-to-four-year-old children's precursor, mapping, and symbolic number comparison skills were compared. Mexican children were recruited from preschools, whereas Canadian children were recruited from

\footnotetext{
${ }^{7}$ In Study 1, some children were observed to spontaneously count the sets of objects during the set reproduction tasks, in addition, performance on the quantity knowledge tasks was found to be moderately correlated with children's verbal counting ability. These findings provided a hint that children may have been relying on their ability to use the number words to determine the quantity of a given set. Thus, the model assessed in the present study included a more direct measure of cardinality (i.e., the Give N task) as opposed to the set reproduction tasks used in Study 1.
} 
daycares. Given the differences in the early educational experiences that children in Canada and Mexico are exposed to, Mexican children were included to assess whether the order of acquisition of the mappings would differ in children with different educational and cultural backgrounds as proposed by Benoit et al. (2013).

Prior studies have shown that parents of Mexican and Latino children have a different conception of early childhood education than North American parents (Reese, 1995). Mexican parents reported that teaching their children morality is beneficial to their academic achievement and thus emphasized this aspect of home experiences more than numeracy or literacy which they believe is the school's responsibility (Reese, 1995). In contrast, Canadian parents report supporting the development of early literacy and numeracy skills from a very young age (e.g., Skwarchuk 2009; Skwarchuk et al., 2014). Parent teaching practices have also been found to differ between Latino and Canadian parents Latino parents tend to use a more formal didactic approach (repetition, and rote memorization) (e.g., Goldenberg, Reese, \& Gallimore, 2000). In contrast, Canadian parents report engaging their preschool-aged children in both formal (e.g., directly teaching their children specific numeracy concepts) and informal practices (e.g., singing the alphabet or number songs) (e.g., Skwarchuk et al., 2014).

With regards to differences in preschool and daycare educational experiences, Mexican children begin formal preschool at age three. School hours are from 9:00 a.m. to 12:30 p.m. Monday to Friday. In contrast, in Ontario, although it is not mandatory, children may be enrolled in Junior Kindergarten at age four (Ontario Ministry of Education, 2014). School hours are from 9:00 to 3:30 p.m., although not all of this time is instructional., Prior to their fourth birthday, many children are enrolled in daycare centers 
or private preschool programs. Children who attend daycare typically attend for the whole day but some children may attend only a few days of the week.

Although in both countries preschools and daycare teachers have similar guidelines for the numeracy skills preschool children are able to develop (Ontario Ministry of Education, 2014; SEP, 2011), the approach taken in each country towards helping children develop these skills differs. In Mexico, teachers are expected to use formal instruction in which children are engaged in activities specifically designed to develop their numeracy skills in order to meet the curriculum standards. In Canada, teachers in both daycare and kindergarten use a play-based approach in which teachers are not expected to directly teach numeracy skills. Thus, Mexican children are presumably explicitly taught number concepts in preschool whereas Canadian teachers are not expected to formally teach children numeracy and literacy concepts.

Despite beginning formal schooling a year before Canadian children, Mexican children have been found to show lower performance than Canadian children on the international PISA assessment at age 15 (OECD, 2012). More research is needed to determine whether Mexican children's underperformance is related to differences in later education (e.g., elementary and middle school) or whether the achievement gap begins to emerge at the preschool age; however, given that Mexican children were enrolled in preschool where number concepts are formally taught, in the present study I hypothesized that Mexican children would score higher than Canadian children on the numeracy measures.

Based on Bialystok's (1992) theory, I expected that children in both countries would learn to associate number words to digits, then number words and quantities and 
finally, digits and quantities. Contrary to Benoit et al, I hypothesized that regardless of early educational experiences and cultural differences, learning the mapping between number words and digits would be easier to learn and would develop first because it does not require the understanding of how counting represents quantity; the mapping between digits and quantities would be the most difficult and be the last mapping to develop because children need to have linked the digit to the corresponding number word, to have an understanding of how the number words represent quantity, and know the counting procedures to determine cardinality for the larger numbers. Furthermore, I did not expect the pattern of relations between precursor, mapping, and symbolic number comparison skills to differ between Mexican and Canadian children.

In order to provide a description of children's preschool and daycare learning environments, in the present study, teachers in both countries were asked to complete a questionnaire on the frequency with which they engaged the children in numeracy and literacy activities. In addition, parents' academic expectations and the frequency with which children were exposed to numeracy and literacy activities at home were assessed by asking parents to complete a questionnaire. Thus, a second goal of the present study was to examine the relationship between parents' reports of the frequency of numeracy and literacy activities conducted at home and children's performance on the math assessments; and to explore the frequency with which children are exposed to early numeracy and literacy activities within their classrooms. Results from the parent and teacher questionnaires are presented in Chapter 6. 


\section{Participants}

\section{Method}

Children. A total of 114 children were recruited for Study 2. Participants were recruited from public preschools in Chihuahua, Mexico and daycare centers in Ottawa, Canada. In Mexico, 60 children (35 girls) were recruited from four local kindergarten schools. Two children refused to participate in the study and one was away during the entire testing period. The final sample therefore consisted of 57 children ( 34 girls). The mean age for the Mexican sample was three years eleven months $(M=47.7$ months, $S D$ $=3.91$, range from 41 to 60 months). In Canada, 54 children (23 girls) were recruited from six daycare centers. Nine children were excluded from the sample: seven because they took part in the pilot testing of the tasks and two because they did not complete one or more tasks. Therefore, the Canadian sample was composed of 45 children (19 girls). The mean age was also three years eleven months $(M=47.7$ months, $S D=5.80$, range from 36 to 60 months $)^{8}$. There was no age difference between the two samples, $t(100)=$ $-.02, p=.98$. The distribution by gender did not differ between samples, $\chi^{2}(1, N=102)=$ $3.06, p=.08$, Cramer's $V=.17$.

The 57 Mexican children were monolingual Spanish speakers. In the Canadian sample, 22 children spoke one or two languages in addition to English at home: 12 children spoke Chinese, seven children spoke French, two children spoke Spanish and one child spoke French and Vietnamese.

Parents. The parents who completed the questionnaire in Mexico were 43 mothers, 9 fathers, and 2 caretakers; in Canada, 33 mothers, 11 fathers, and 1 caretaker completed the questionnaire. Information on parent education was available for 54 and 53

\footnotetext{
${ }^{8}$ Frequency distribution for participants' age are presented in Appendix D.
} 
Mexican mothers and fathers respectively; and for 44 and 40 Canadian mothers and fathers respectively. Table 4.1 presents the number of parents and their reported highest level of education for both countries. Canadian parents were significantly more highly educated than Mexican parents, for mothers $\chi^{2}(1, N=98)=31.82, p<.001$, Cramer's $V$ $=.57$, and for fathers $\chi^{2}(1, N=93)=28.49, p<.001$, Cramer's $V=.55$. In the Mexican sample, $55 \%$ of the mothers and $58 \%$ of the fathers had not studied beyond high school; in the Canadian sample, this was true for only $2 \%$ of the mothers and $5 \%$ of the fathers. Table 4.1. Highest level of education for Mexican and Canadian parents.

\begin{tabular}{lcccc}
\hline & \multicolumn{2}{c}{ Mexico } & \multicolumn{2}{c}{ Canada } \\
\hline Level of Education & Mother & Father & Mother & Father \\
\hline Less than high school & 14 & 18 & 0 & 0 \\
High school & 16 & 13 & 1 & 2 \\
College graduate & 14 & 6 & 3 & 5 \\
University graduate & 7 & 14 & 15 & 11 \\
Post graduate & 3 & 2 & 25 & 22 \\
\hline
\end{tabular}

Teachers. Table 4.2 summarizes the number of participating preschools or daycares, and the number of teachers and children. Five Mexican preschool teachers completed the teacher questionnaire. Each teacher was responsible for a single class: In one of the four preschools there were two groups of children. All the Mexican teachers had obtained a university degree.

In Canada, 15 daycare teachers from the six daycares completed the questionnaire. Daycares typically have several teachers working with a group of children 
at different times during the day. In the present study, the number of teachers who responded the questionnaire from a single daycare ranged from one to four. Information on highest level of education was available for 14 teachers, from these, 8 were college graduates, 3 had a university degree, and 3 had post-graduate degrees.

Table 4.2. Distribution of teachers who completed the questionnaire and the number of children from each preschool or daycare.

\begin{tabular}{|c|c|c|c|c|}
\hline \multirow[t]{2}{*}{ Preschool/Daycare } & \multicolumn{2}{|c|}{ Mexico } & \multicolumn{2}{|c|}{ Canada } \\
\hline & Teachers & Children & Teachers & Children \\
\hline 1 & 1 & 8 & 3 & 4 \\
\hline 2 & 1 & 12 & 4 & 3 \\
\hline 3 & 1 & 15 & 2 & 9 \\
\hline 4 & 2 & 22 & 3 & 7 \\
\hline 5 & -- & -- & 2 & 16 \\
\hline 6 & -- & -- & 1 & 6 \\
\hline Totals & 5 & 57 & 15 & 45 \\
\hline
\end{tabular}

\section{Materials}

All children completed the same set of 10 measures. For the Mexican children all the measures were translated and children were tested in Spanish by the author who is Mexican.

Verbal counting. The purpose of this task was to assess the highest number up to which children knew the counting words. To make the task interesting, the experimenter told the child that a sheep puppet named Dolly needed their help to learn how to count 
because she only knew the number one. The task began when Dolly the sheep said the number "one," the experimenter then asked the child "Do you know which number comes after one?" The score on this task was the highest number up to which children could count correctly; when a child realized he/she had made a mistake and corrected it, he/she was permitted to continue.

Mapping tasks. Six tasks were designed to assess children's ability to map between number representations (i.e., number-word-to-quantity, quantity-to-numberword, digit-to-quantity, quantity-to-digit, number-word-to-digit, digit-to-number-word). All the mapping tasks were presented on an i-pad and consisted of 18 test trials each (see Figure 4.1 for examples of the stimuli). On each trial children were presented with a target number representation (i.e., a number word "three", a digit " 3 " or a quantity “पप्") and were given two options (either two digits, quantities, or number words) one of which matched the target number representation. Children were asked to choose the corresponding number by pressing one of two response buttons located beneath the two options. There was no time limit for the trials.

When the children were exposed to number words, these were presented by two cartoon characters (i.e., a penguin and a beaver) and children were permitted to listen to them as many times as they required. On the tasks that involved non-symbolic quantities and when these were the response options, the quantities were sets of colorful rectangles (blue, green, purple, yellow, brown, or pink) enclosed within a same-colored rectangular border; when the quantities were the target number they were always black, also enclosed within a black border. The size of the quantity rectangles was negatively correlated with the number and ranged in size from $15.35 \mathrm{~cm}^{2}$ for the largest to $1.31 \mathrm{~cm}^{2}$ for the smallest. 
On these tasks, children were not instructed to but were allowed to count the set(s) if they wished to do so. When the target numbers were digits, these were presented in black and enclosed within a black rectangle, when the digits were the response options they were presented in different colors (blue, green, purple, yellow, brown, or pink) enclosed within a same colored rectangle.

The numbers that were assessed were 1-9 with two trials for each target number. The numbers were categorized as small (i.e., 1-3), medium (i.e., 4-6), and large (i.e., 7-9). In order to make the matching tasks comparable, the six tasks included the same options of number pairs for each target number (presented in Appendix E). The tasks were set up so that the small numbers were always presented first, followed by the medium and finally the large numbers; however, within each category of numbers, these were presented in random order.

To familiarize the children with the mapping task procedure, before beginning each task they were presented with practice trials in which they had to match colorful geometric figures to either the name of the color or a figure that was the same color and shape as the target. In order to begin the test trials, children had to successfully complete three consecutive practice trials. During the practice trials children were provided corrective feedback to ensure that they understood the nature of the mapping task; however, during the test trials no feedback was provided although children were verbally encouraged at all times. 
Figure 4.1. Examples of the mapping task stimuli.

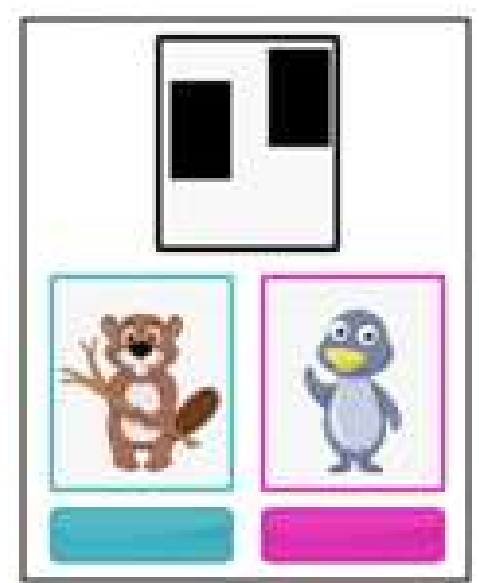

Quantity-to-number-word

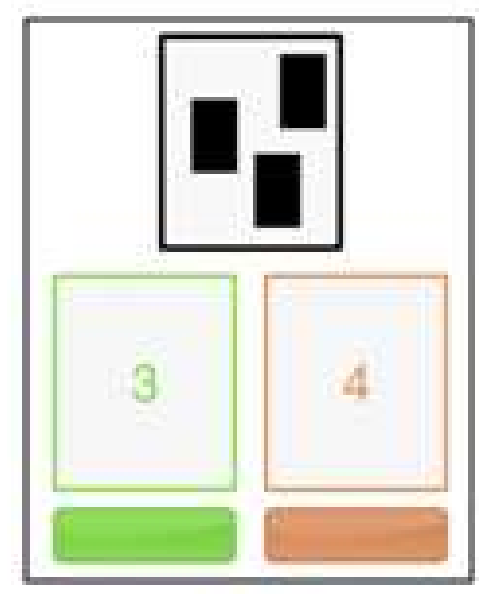

Quantity-to-digit

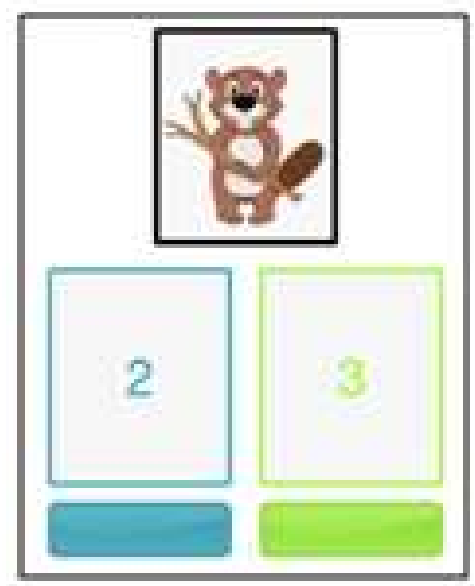

Number-word-to-digit

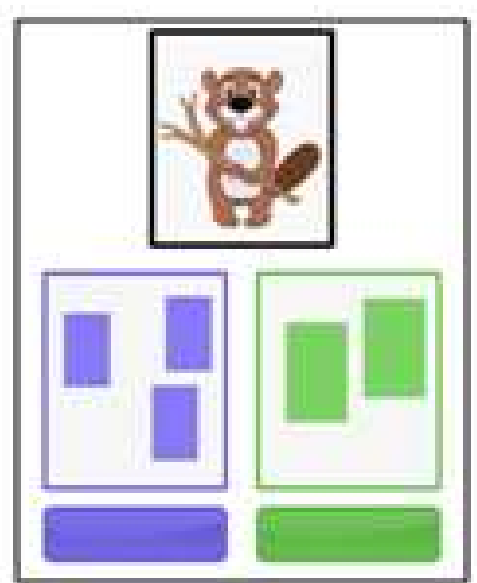

Number-word-to-quantity

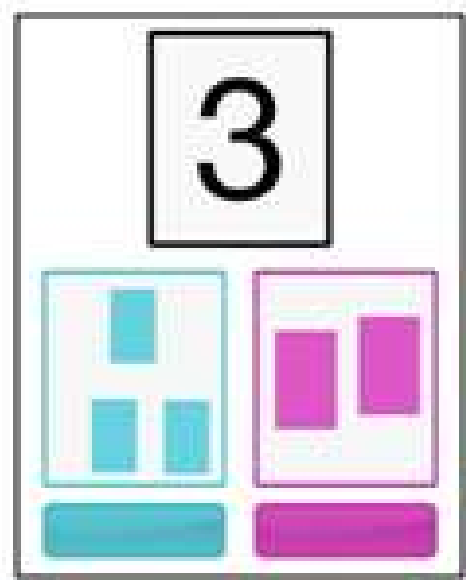

Digit-to-quantity

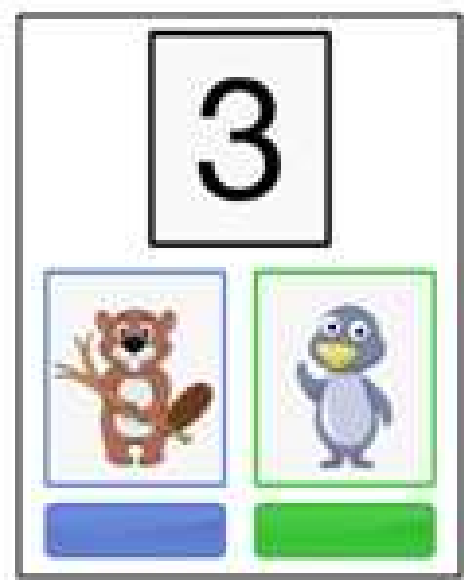

Digit-to-number-word 


\section{Quantity-to-number-word.}

Practice trials. Children were shown a figure (i.e., a star, a square or a triangle) which could be purple, blue, orange or green on the upper middle part of the screen. A penguin and a beaver appeared on the bottom left and right sides of the screen with a response button below each cartoon character. Children were told that when they touched the penguin and the beaver, each would say a color and that they had to decide which cartoon said the color that was the same as the geometric figure above. Once children had listened to both characters they were told to press the response button below the character that had said the correct color.

Test trials. Children were shown a set of black rectangles on the upper middle part of the screen and a penguin and a beaver on the bottom left and right sides of the screen. Children were told that when touched, the penguin and the beaver would each say a number word and that the child had to decide which number word was the same as the set of squares shown above and press the appropriate response button.

\section{Number-word-to-quantity.}

Practice trials. A picture of a beaver cartoon was presented on the top middle part of the screen and children were instructed to touch the image and listen to the color the character would say. Children's attention was then directed to two geometric figures that were presented on the bottom left and right sides of the screen and they were then asked to decide which of the two was of the same color the beaver had said; once the child had decided, he/she was asked to touch the response button below the corresponding figure.

Test trials. Children were presented with a cartoon beaver on the upper middle part of the screen and two sets of different color rectangles on the bottom left and right 
part of the screen. The experimenter told the child that when touched, the beaver would say a number word and that he or she had to decide which set had the same number of rectangles and press the corresponding response button.

\section{Quantity-to-digit.}

Practice trials. Children were presented with a geometric figure on the top middle part of the screen and two figures on the bottom left and right sides of the screen one of which was the same as the figure on the top. The experimenter asked the child to decide which of the two figures was the same as the sample figure on top and press the corresponding response button.

Test trials. Children saw a set of black rectangles on the top middle part of the screen and two colorful Arabic digits on the left and right bottom sides of the screen. The experimenter asked the children to decide which of the two Arabic digits was the same as the number of rectangles on the upper part of the screen and to then press the appropriate response button.

\section{Digit-to-quantity.}

Practice trials. Children were presented with a geometric figure on the top middle part of the screen and two figures on the bottom left and right sides of the screen one of which was the same as the figure on the top. The experimenter asked the child to decide which of the two figures was the same as the sample figure on top and press the corresponding response button.

Test trials. Children saw a black Arabic digit on the top middle part of the screen and two sets of colorful rectangles on the left and right bottom sides of the screen. The 
experimenter asked the children to decide which of the two sets had the same number of rectangles as the number on the top and to press the appropriate response button.

\section{Number-word-to-digit.}

Practice trials. Children were presented with a cartoon beaver on the top middle part of the screen and two different color geometric figures. Children were instructed to touch the beaver and listen to the color it said, they then had to decide which figure was the same color and press the corresponding response button.

Test trials. Children saw a cartoon beaver on the top middle part of the screen and two colorful Arabic digits on the bottom left and right sides of the screen. The experimenter instructed the children to touch the beaver, listen to the number word, decide which digit was the same as the number word and press the appropriate response button.

\section{Digit-to-number-word.}

Practice trials. Children were presented with a colorful geometric figure on the middle upper part of the screen and two cartoon characters (i.e., a penguin and a beaver) on the bottom left and right sides of the screen. The experimenter asked the child to touch each of the cartoons and listen to the colors each said and choose the one that had said the color that was the same as the figure by pressing the corresponding response button.

Test trials. Children saw a black Arabic digit on the upper middle side of the screen and the two cartoon characters (i.e., a beaver and a penguin) on the bottom left and right sides of the screen. The experimenter asked the children to touch each cartoon character, listen to the number word and decide which character had said the number that was the same as the target digit by pressing the appropriate response button. 
Verbal comparison. The purpose of this task was to assess children's ability to mentally compare two number words and determine which represented the greater quantity. Children completed a total of 20 trials in which they were told that the puppets Dolly the sheep and Belle the cow had gone shopping for fruits and vegetables. Children were told the amount of food and each one had bought (e.g., "Dolly bought five apples and Belle bought eight apples") and the experimenter would then ask the child to point to the puppet that had bought more fruit by saying: "Who bought more X?" No feedback was provided as to the correctness of the answer but children were verbally encouraged at all times. The score on this task was the total number of correct responses.

The numbers assessed included 1-9. The item pairs included distances of 1 and 3 . For half of the items the larger number was presented first. The comparison pairs were presented in one of two predetermined orders (to which children were randomly assigned) and each pair was presented twice within the task (stimuli are listed in Appendix F).

Give N. Following Wynn (1992), children were presented with 20 colored $3 \times 3 \times 3 \mathrm{~cm}$ foam cubes (either blue or green), a red plastic bowl, and a puppet which could be a cow named Belle or a sheep named Dolly. The task began with the experimenter explaining to the child that he or she needed to give the puppet some cubes and place them in the bowl. The experimenter initially asked the child to place 1 cube in the bowl, if the child succeeded he or she was asked for 2 , if the child failed to correctly place 2 cubes in the bowl, the experimenter again asked for 1 , if the child was successful, the experimenter asked for 2 cubes again, if the child succeeded the experimenter would ask for the next number (i.e., 3) and the task continued in this way until the child failed to 
correctly create a set with a determined number of cubes two out of three times or the child reached the highest number assessed which was 6 .

KeyMath-R Numeration Subtest. This test was used as a measure of early numeracy knowledge. Children were randomly assigned to complete either version A or B. The items included 10 questions that assessed children's knowledge of the counting string, their ability to count sets of objects, recognize written number symbols, and to understand the ordinal meaning of numbers (Connolly, 2000). Children received one point for every correct response; following the test instructions, testing was stopped when the child failed on three consecutive items. All children in the present study were randomly assigned to complete either version A or B of the Numeration Subtest ${ }^{9}$. This decision was made because Canadian children would also participate in Study 3 which involved post-testing. For consistency, the Mexican children also completed one of the two versions of the subtest.

Parent questionnaires. The parent questionnaire included 13 questions about academic expectations: numeracy expectations (6), and literacy expectations (7). Parents responded on a five-point scale which ranged from unimportant to extremely important. The questionnaire also included 24 questions about home numeracy (13) and literacy practices (11). Parents responded on a five-point scale that ranged from rarely or never to most days of the week. Detailed information about the Parent and Teacher Questionnaires is provided in Chapter 6 .

Teacher questionnaire. The teacher questionnaire included questions about the teacher's highest level of education, the frequency with which they engaged children in

\footnotetext{
${ }^{9}$ Version A and version B of the KeyMath Numeration Subtest were designed to be equivalent (Conolly, 2000).
} 
numeracy (19) and literacy activities (10). Teachers responded on a five-point scale that ranged from rarely or never to most days of the week. In addition, teachers were asked about which activities that included counting were the most common and about the number of books present in their classrooms.

\section{Procedure}

Recruitment. In Mexico and Canada, permission to conduct testing was obtained from the daycare and preschool directors and teachers by providing them with a written description of the study. Permission from Canadian parents was obtained by sending home a letter describing the nature of the study. In Canada, the parent questionnaires were sent home and returned to the daycare with the consent form. Canadian parents were also given the option to complete the questionnaire online (only one parent responded via the electronic version). Mexican parents were asked to attend a meeting with the preschool director, their children's teacher, and the experimenter; during the meeting the experimenter explained the purpose and procedure of the study and provided them with the written description, the consent form and the parent questionnaire. Parents who agreed to participate completed the consent forms and questionnaires during the meeting. In both countries, children whose parents granted informed consent were asked if they wished to participate before they were tested; those who agreed were tested individually in a quiet area of their daycare or preschool. Children were awarded stickers to thank them for their participation. Children who chose to stop testing were also thanked and received their sticker.

Children. Pilot testing showed that the iPad mapping and the verbal comparison tasks were demanding for our young participants, therefore, the tasks were presented in a 
fixed order in which children were never presented with two consecutive mapping tasks and the verbal comparison task was divided into two parts. The order of presentation of the tasks was: 1.verbal counting, 2. mapping, 3. Give N, 4. mapping, 5. verbal comparison, 6. mapping, 7. mapping, 8. KeyMath, 9. mapping, 10. verbal comparison, and k) mapping. Although the mapping tasks were not presented consecutively, there were six predetermined sequences for the order in which they were presented to the children; these sequences are presented in Appendix G.

In Mexico, all the children completed the 10 tasks in a single 30 -minute session. In Canada, most children required two 30-minute sessions to complete the tasks. This difference was may have reflected differences in children's experience: In Mexico the children were accustomed to remaining seated and focused on tasks because they had been in a formal school system for seven months.

Teachers. Teachers in both Mexico and Canada were interviewed briefly by an experimenter and when their schedule allowed it, they completed the questionnaire with the experimenter; when this was not possible the teachers took the questionnaire and returned it at a later time.

\section{Results}

I first examine children's performance on all tasks. Because Mexican children had been enrolled in a formal school system in which they would presumably be more exposed to math activities, I expected their mean scores to be higher. Multi-group path analyses were then used to examine whether the relationships among early numeracy skills found in Study 1 would show a similar pattern in children from both countries. Overall mean percent correct for all tasks are shown in Table 4.3. 


\section{Verbal Counting}

Children in Mexico could verbally count on average to 8 with a range of 1-19 whereas Canadian children on average could count to 19 with a range of 2-100. Figure 4.2 shows the percentage of children who could count up to a given number in both countries. Mexican children performed similarly to Canadian children only up to the number 5; from number 6 onwards there was a marked decline in the percentage of Mexican children who could count correctly. Eighty percent of Canadian children could count correctly to number 10 while this was true for only $42 \%$ of the Mexican children. Furthermore, only one Mexican child was able to count correctly up to 19 while this was true for $17(38 \%)$ of Canadian children. 
Table 4.3. Description of the tasks used in Study 2.

\begin{tabular}{|c|c|c|c|c|c|c|c|}
\hline \multirow[t]{2}{*}{ Task } & \multirow[t]{2}{*}{ Description } & \multirow{2}{*}{$\begin{array}{l}\text { Trials } \\
\text { (Max) }\end{array}$} & \multirow[t]{2}{*}{ Time } & \multicolumn{2}{|c|}{ Reliability } & \multicolumn{2}{|c|}{$\begin{array}{l}\text { Overall Mean Percent Correct } \\
(S D)\end{array}$} \\
\hline & & & & Canada & Mexico & Canada & Mexico \\
\hline Verbal counting & $\begin{array}{l}\text { Participants were asked to } \\
\text { count as high as they could. }\end{array}$ & 1 & 1 & & & $19.40(19.56)$ & $8.32(3.30)$ \\
\hline Mapping Tasks & & & & & & & \\
\hline $\begin{array}{l}\text { Quantity-to- } \\
\text { number-word }\end{array}$ & $\begin{array}{l}\text { Children had to match a } \\
\text { quantity to the corresponding } \\
\text { number word. }\end{array}$ & 18 & 5 & .66 & .70 & $76.1(15.7)$ & $66.5(18.4)$ \\
\hline $\begin{array}{l}\text { Number-word-to- } \\
\text { quantity }\end{array}$ & $\begin{array}{l}\text { Children had to match a } \\
\text { number word to the } \\
\text { corresponding quantity. }\end{array}$ & 18 & 5 & .64 & .63 & $75.4(15.5)$ & $63.7(17.2)$ \\
\hline Quantity-to-digit & $\begin{array}{l}\text { Children had to match a } \\
\text { quantity to the corresponding } \\
\text { digit. }\end{array}$ & 18 & 5 & .65 & .43 & $70.5(16.9)$ & $55.9(15.0)$ \\
\hline Digit-to-quantity & $\begin{array}{l}\text { Children had to match a digit } \\
\text { to the corresponding quantity. }\end{array}$ & 18 & 5 & .75 & .53 & $68.9(19.8)$ & $58.7(16.3)$ \\
\hline $\begin{array}{l}\text { Number-word-to- } \\
\text { digit }\end{array}$ & $\begin{array}{l}\text { Children had to match a } \\
\text { number word to the } \\
\text { corresponding digit. }\end{array}$ & 18 & 5 & .81 & .74 & $89.5(14.9)$ & $68.1(19.8)$ \\
\hline $\begin{array}{l}\text { Digit-to-number- } \\
\text { word }\end{array}$ & $\begin{array}{l}\text { Children had to match a digit } \\
\text { to the corresponding number } \\
\text { word. }\end{array}$ & 18 & 5 & .84 & .78 & $87.3(17.1)$ & $67.0(21.6)$ \\
\hline $\begin{array}{l}\text { Verbal number } \\
\text { comparison }\end{array}$ & $\begin{array}{l}\text { Children had to decide which } \\
\text { was the larger of two verbal } \\
\text { number words. }\end{array}$ & 20 & 4 & .86 & .75 & $69.7(24.0)$ & $47.7(20.4)$ \\
\hline
\end{tabular}




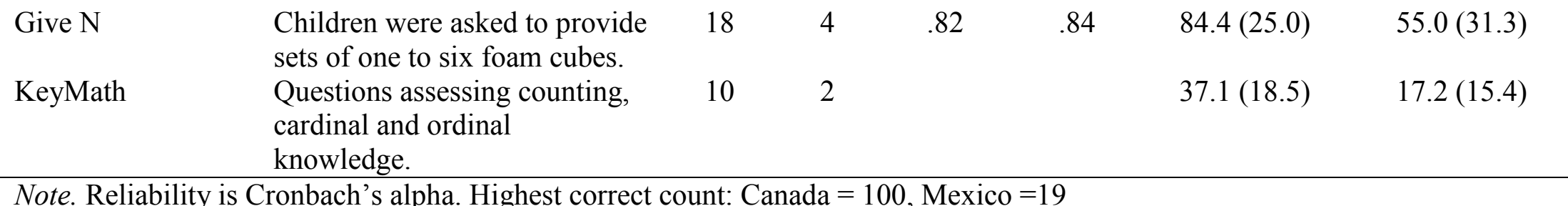


Figure 4.2. Percentage of children who counted successfully to each number (i.e., highest correct count).

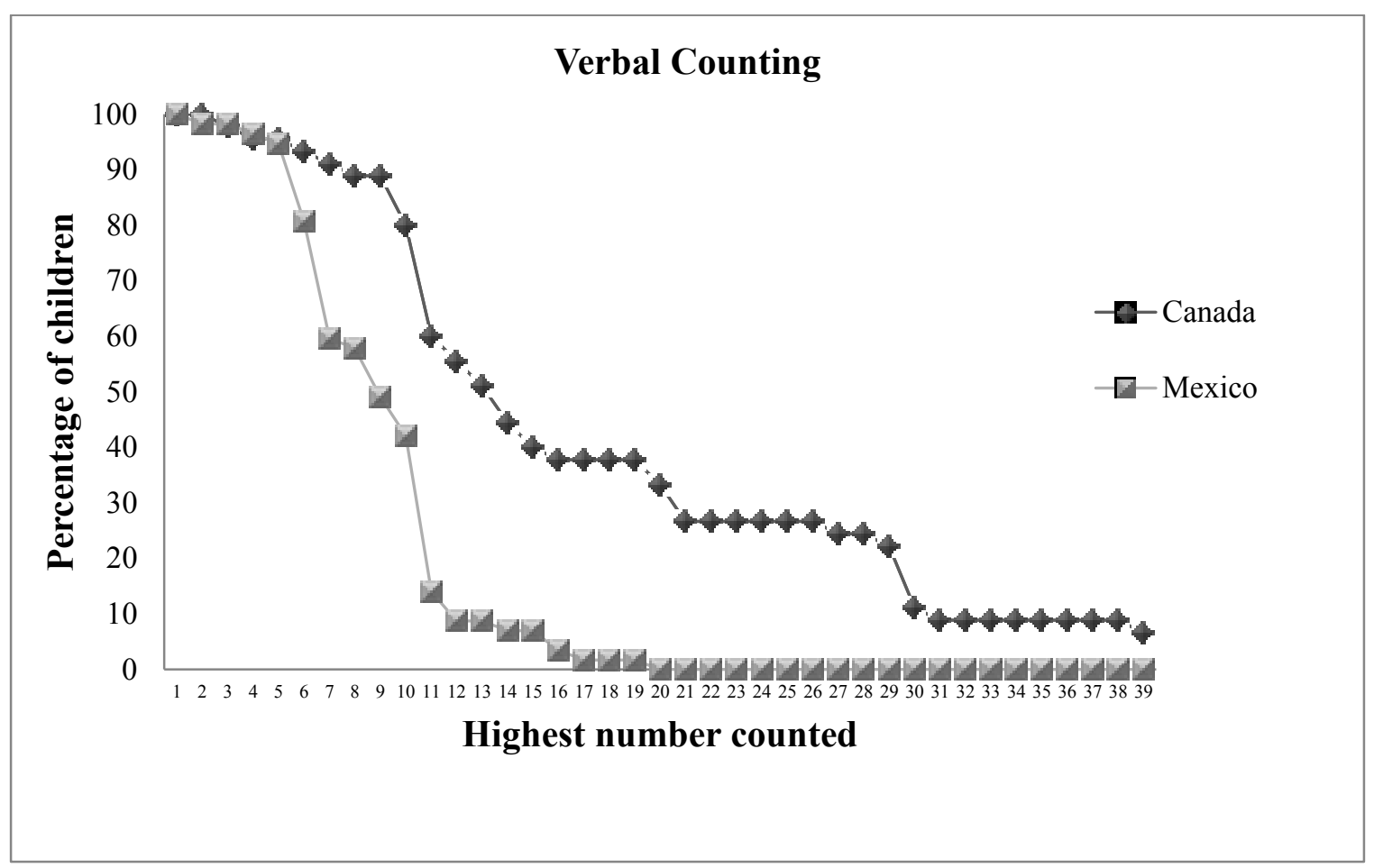




\section{Mapping tasks}

Preliminary analyses showed no effect of direction of mapping (see Appendix H), thus, performance on the mapping tasks was analyzed collapsing across mapping direction in a 3 (task: quantities and digits, quantities and words, words and digits) $\mathrm{x}$ 3(size: small, medium, large) x 2 (country) mixed ANOVA. Means collapsing across mapping direction are presented in Table 4.4, results are summarized in Table 4.5. Note that the maximum score in each condition was 6 .

As shown in Table 4.5, all main effects and interactions were statistically significant. Canadian children performed better than Mexican children (4.68 vs 3.80). Overall, performance was better on the word-digit tasks and worse on the digit-quantity tasks (word-digit: 4.68; word-quantity: 4.23; digit-quantity: 3.81; Bonferroni pairwise comparisons all $p \mathrm{~s}<.001)$. Children also performed better on the small, and worse on the large set size (small: 4.83; medium: 4.19; large: 3.69; Bonferroni pairwise comparisons all $p \mathrm{~s}<.001)$.

Table 4.4. Mapping task means by set size and country

\begin{tabular}{lcccccc}
\hline Task & \multicolumn{5}{c}{ Mexico $(n=57)$} & \multicolumn{3}{c}{ Canada $(n=45)$} \\
\cline { 2 - 7 } & Small & Medium & Large & Small & Medium & Large \\
\cline { 2 - 7 } & $M(S D)$ & $M(S D)$ & $M(S D)$ & $M(S D)$ & $M(S D)$ & $M(S D)$ \\
\hline Digit-quantity & $3.82(1.34)$ & $3.33(1.10)$ & $3.15(0.92)$ & $4.94(1.18)$ & $4.10(1.24)$ & $3.50(1.19)$ \\
Word-quantity & $4.86(1.14)$ & $3.71(1.27)$ & $3.15(1.08)$ & $5.40(0.81)$ & $4.68(1.28)$ & $3.56(1.11)$ \\
Digit-word & $4.50(1.24)$ & $3.90(1.42)$ & $3.75(1.32)$ & $5.46(0.95)$ & $5.42(0.95)$ & $5.03(1.19)$
\end{tabular}


Table 4.5. Statistics for the Task by Size by Country ANOVA.

\begin{tabular}{lcccc}
\hline Factor & $d f$ & $F$ & $M S E$ & $\eta_{p}{ }^{2}$ \\
\hline Country & 1,100 & $27.77^{* * *}$ & 6.28 & .22 \\
Task & 2,200 & $59.82^{* * *}$ & 0.95 & .37 \\
Task x country & 2,200 & $8.47^{* * *}$ & 0.95 & .08 \\
Size & 2,200 & $121.14^{* * *}$ & 0.81 & .55 \\
Size x country & 2,200 & $3.81^{*}$ & 0.81 & .04 \\
Task x size & $3.71,371$ & $14.87^{* * *}$ & 0.67 & .13 \\
Task x size x country & 4,400 & $3.87^{* *}$ & 0.62 & .04 \\
\hline
\end{tabular}

There was a significant task by country interaction (Figure 4.3). For the Canadian children, as in Study 1, there were significant differences between all three tasks. In contrast, for Mexican children, the difference in performance between word-quantity and word-digit tasks was not significant and digit-quantity performance was worse than both.

The significant size by country interaction (Figure 4.4) showed that although in both countries there were significant differences among all three set sizes (Bonferroni corrected pairwise comparisons), across tasks, the difference between the medium and large set sizes for the Mexican children (difference medium - large $=0.30$ ) was smaller than that for the Canadian children (difference medium - large $=0.70), F(1,100)=7.10$, MSE $=2.33, p=.009, \eta_{p}^{2}=.07$. 
Figure 4.3. Task x Country interaction. Error bars are 95\% C.I.s based on the MSE for the Country x Task interaction.

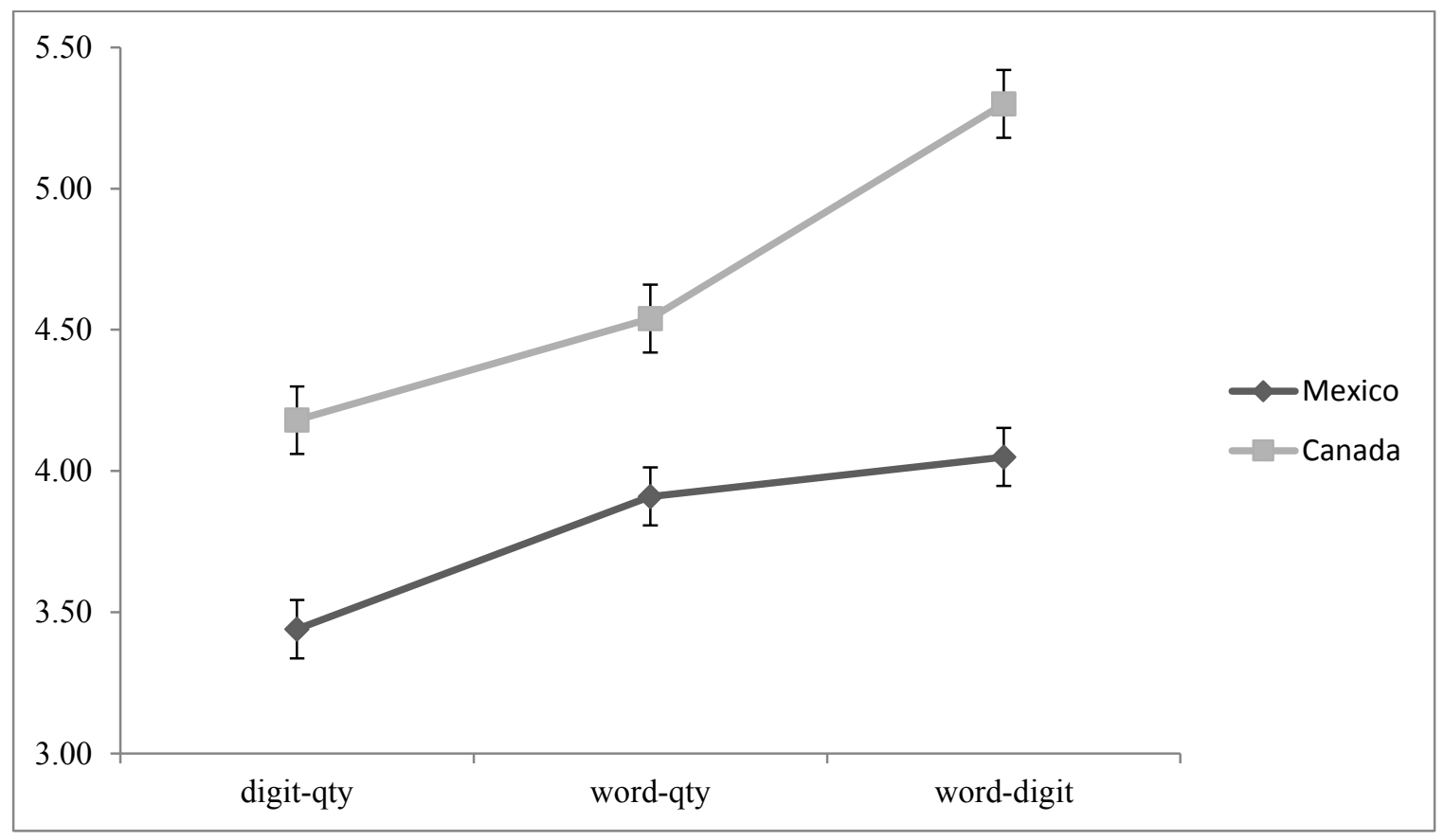

Figure 4.4. Performance by set size and country. Error bars are 95\% CIs based on the MSE for the size by country interaction.

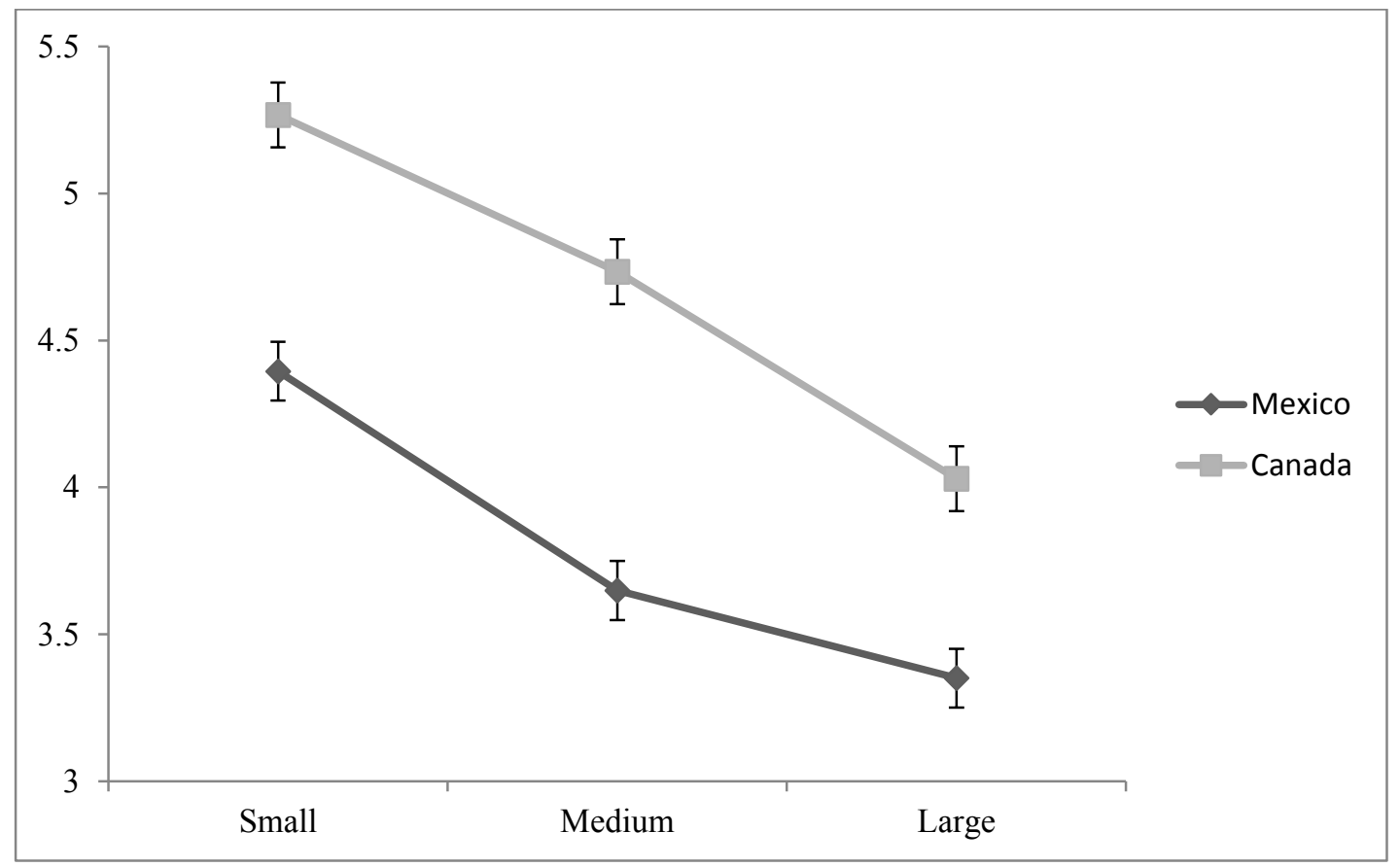


The significant task by size interaction was of less interest given the focus on the difference in performance between children in the two countries, however, pairwise comparisons indicated that at the small set size there was no difference in performance between the quantity-word and word-digit tasks, while at the large set size there was no difference in performance between quantity-digit and quantity-word tasks.

Importantly, there was a significant Task x Country x Size interaction ${ }^{10}$, as shown in Figure 4.5. At the small set size $(1-3)$, Canadian children showed no differences in performance between the word-digit and word-quantity tasks; performance on both of these tasks was significantly better than performance on the digit-quantity task. In contrast, Mexican children's performance differed among the three types of tasks: they performed better on the word-quantity than the word-digit task, and, as was the case with the Canadian children, performed more poorly when mapping digits and quantities. At the medium set size $(4-6)$, for the Canadian children there was a significant difference in performance among the three mapping tasks. Mexican children also showed higher performance on the word-digit mapping than on the digit-quantity tasks, but in contrast to the Canadian children, there was no difference in performance between Word-quantity and Word-digit mapping. At the large set size (7 - 9), the pattern of performance was similar across groups: performance was better on the word-digit mapping than on both word-quantity and digit-quantity mapping, with no difference in performance between the two symbol-quantity tasks.

${ }^{10}$ Canadian children showed above chance (i.e., $50 \%$ ) on average, on all tasks at each of the three set sizes (all $p \mathrm{~s}<.01$ ); in contrast, Mexican children's average performance did not differ from chance on the digit-quantity $(M=3.15)$ and word-quantity $(M=3.15)$ tasks at the large set size, $t(56)=1.23, p=.22$, and $t(56)=1.04, p=.30$ respectively. 
Figure 4.5. Performance on the three tasks by set size for both countries. Error bars are 95\% Confidence Intervals based on the MSE for the Task x Size x Country interaction.
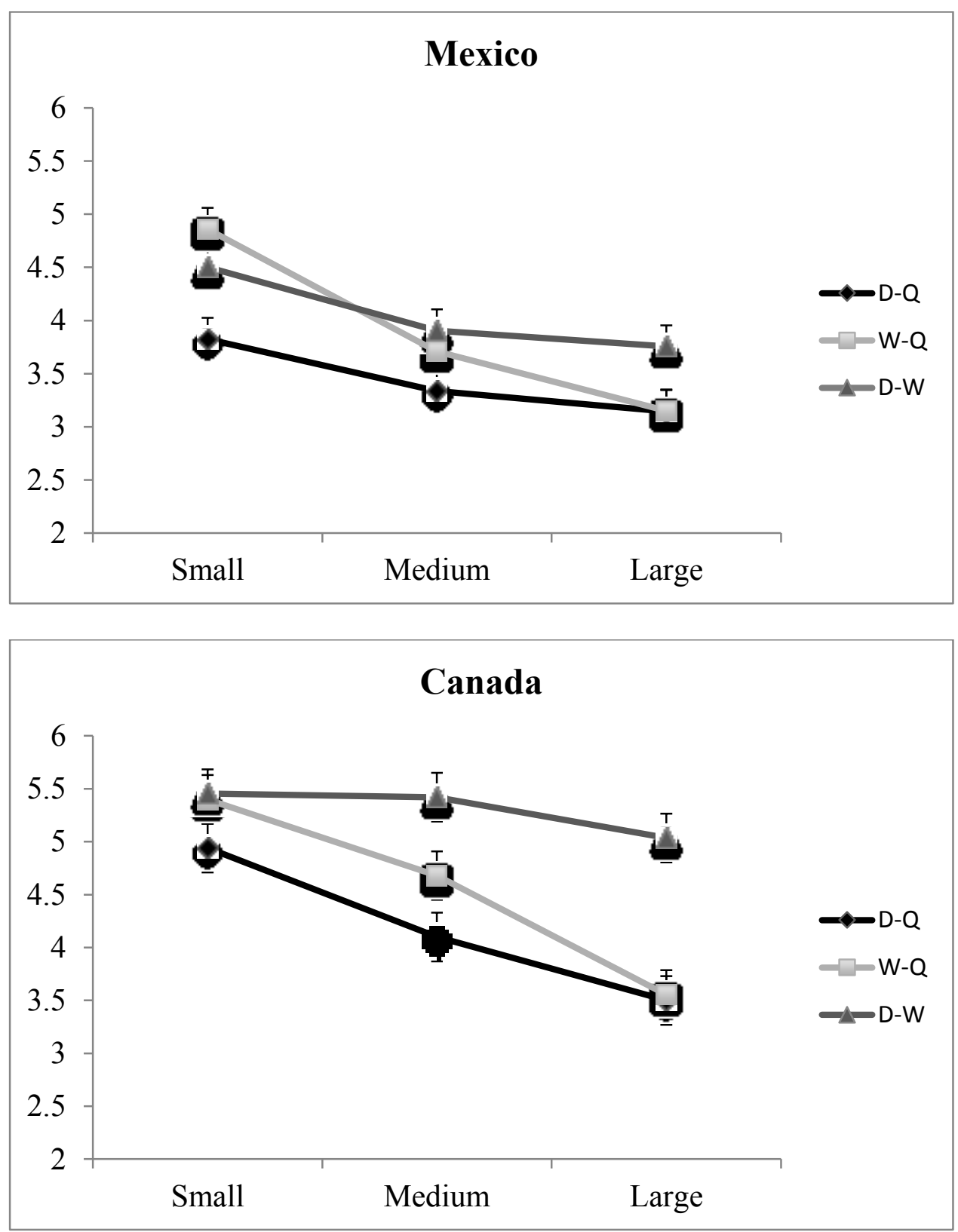

In summary, Canadian children had better mapping skills than the Mexican children. Replicating the results of Study 1, Canadian children's performance was better 
on the word-digit mapping and worse on the digit-quantity mapping across the three set sizes. Mexican children showed a small but significant advantage on the word-quantity task over the word-digit task at the small set size; at the medium set size, performance did not differ between word-digit and word quantity but at the large set size performance on the symbol-symbol mapping was better than on the symbol-quantity mappings for which performance was not different from chance.

The finding that digit-word mapping was the only task on which Mexican children performed above chance at the large set size is important because it shows that despite the differences in the pattern of results between Mexican and Canadian samples, the groups were similar in showing better ability to map between number words and digits (symbol-symbol mapping) than between symbols and quantities, as in Study 1. Finally, as in Study 1, both groups showed worse performance on the digit-quantity mapping than on the word-quantity mapping task.

\section{Give N}

As shown in Table 4.1, Mexican children's performance was significantly worse than that of Canadian children, $t(100)=-5.15, p<.001$. Mexican children could on average successfully produce sets of 3 cubes; Canadian children had better understanding of cardinality and could on average successfully produce sets of 5 cubes.

\section{KeyMath}

Children were randomly assigned to complete either version A (Mexico $n=27$; Canada $n=19$ ) or B. Table 4.6 summarizes performance on both versions by country. Children's performance was analyzed in a 2 (country: Mexico, Canada) by 2 (version: A, B) ANOVA. Overall, Canadian children performed better than the Mexican children, $F$ 
$(1,98)=38.43, M S E=2.61, p=.000, \eta^{2} \mathrm{p}=.28$; and performance in both countries was better on version $\mathrm{A}$ than version $\mathrm{B}, F(1,98)=9.41, p=.003, \eta^{2}{ }_{\mathrm{p}}=.09$. Because there were differences between versions, performance was standardized within version and these z-scores were used in further analyses.

Table 4.6. Performance on both versions of the KeyMath by country.

\begin{tabular}{lcccccc}
\hline & \multicolumn{3}{c}{ Mexico } & \multicolumn{3}{c}{ Canada } \\
\cline { 2 - 7 } & $n$ & $M$ & $S D$ & $N$ & $M$ & $S D$ \\
\hline KeyMath A & 27 & 2.41 & 1.53 & 19 & 4.11 & 1.63 \\
KeyMath B & 30 & 1.10 & 1.30 & 26 & 3.42 & 1.98 \\
\hline
\end{tabular}

\section{Verbal number comparison}

As detailed in the method section, the verbal comparison task included distances of 1 and 3 (10 trials each) and all children completed the 20 trials. Table 4.7 presents the means for the Mexican and the Canadian children by distance. Performance on this task was analyzed in a 2 (distance: 1,3$)$ x 2 (country: Mexico, Canada) mixed ANOVA. The only significant effect was that of country, $F(1,100)=24.68, M S E=9.72, p=.000, \eta^{2}{ }_{p}=$ .20, overall, Canadian children outperformed their Mexican peers. Performance was above chance for the Canadian children for both distances ( $p$ s $<.001)$. In contrast, Mexican children's performance was not different from chance at either distance ( $p$ s $>$ $.33)$. 
Table 4.7. Performance on verbal comparison by distance and country (maximum score is 10).

\begin{tabular}{lcccc}
\hline Comparison distance & \multicolumn{2}{c}{ Mexico } & \multicolumn{2}{c}{ Canada } \\
\cline { 2 - 5 } & $M$ & $S D$ & $M$ & $S D$ \\
\hline Distance 1 $(\max .=10)$ & 4.72 & 2.19 & 6.67 & 2.49 \\
Distance 3 $(\max .=10)$ & 4.82 & 2.44 & 7.24 & 2.61 \\
\hline
\end{tabular}

\section{Relations among numeracy measures}

Before analyzing the correlations, the two mapping tasks for each direction were averaged. Table 4.8 presents the zero-order correlations among the numeracy tasks, as well as the means and standard deviations for the combined mapping tasks in both samples. The correlation table with the six mapping tasks can be found in Appendix J. In both samples performance on the numeracy measures was correlated with age, the only exception being the verbal comparison task in the Mexican sample. Partial correlations among the numeracy measures controlling for age are presented in Table 4.9. 
Table 4.8. Zero order correlations among tasks and home numeracy practices (mapping combined).

Mexican sample above the diagonal, Canadian sample below the diagonal.

\begin{tabular}{|c|c|c|c|c|c|c|c|c|c|}
\hline & Age & Gender & $\begin{array}{l}\text { Verbal } \\
\text { Count }\end{array}$ & $\begin{array}{l}\text { Digit- } \\
\text { quantity }\end{array}$ & $\begin{array}{l}\text { Word- } \\
\text { quantity }\end{array}$ & Word-digit & Give N & KeyMath & $\begin{array}{l}\text { Verbal } \\
\text { Comp. }\end{array}$ \\
\hline Age & -- & & $.49 * *$ & $37 * *$ & $.37 * *$ & $.33 *$ & $.34 * *$ & $.38^{* *}$ & .19 \\
\hline Gender & & -- & $-.27 *$ & -.17 & -.17 & -.14 & $-.28 *$ & -.22 & .08 \\
\hline Verbal count & $.41^{* *}$ & .19 & -- & $.62 * *$ & $.53 * *$ & $.51 * *$ & $.69 * *$ & $.72 * *$ & $.44^{* *}$ \\
\hline Digit-quantity & $.47 * *$ & -.22 & $.38^{*}$ & -- & $.55 * *$ & $.66^{* *}$ & $.59 * *$ & $.70 * *$ & $.42 * *$ \\
\hline Word-quantity & $.51 * *$ & -.23 & $.48 * *$ & $.80 * *$ & -- & $.66^{* *}$ & $.61 * *$ & $.68 * *$ & $.64 * *$ \\
\hline Word-digits & $.44^{* *}$ & -.12 & $.44 * *$ & $.67 * *$ & $.67^{* *}$ & -- & $.56^{* *}$ & $.69 * *$ & $.56^{* *}$ \\
\hline Give N & $.58 * *$ & -.27 & $.45^{* *}$ & $.57 * *$ & $.69 * *$ & $.58 * *$ & -- & $.74 * *$ & $.50 * *$ \\
\hline KeyMath & $.48^{* *}$ & -.17 & $.57 * *$ & $.66^{* *}$ & $.76^{* *}$ & $.58 * *$ & $.71 * *$ & -- & $.60 * *$ \\
\hline Verbal Comp. & $.62 * *$ & .05 & $.55^{* *}$ & $.73 * *$ & $.77 * *$ & $.60 * *$ & $.63 * *$ & $.67 * *$ & -- \\
\hline Canada $M(S D)$ & & & & $12.54(2.99)$ & $13.62(2.45)$ & $15.91(2.62)$ & & & \\
\hline $\operatorname{Mexico} M(S D)$ & & & & $10.31(2.51)$ & $11.72(2.94)$ & $12.16(3.43)$ & & & \\
\hline
\end{tabular}


Table 4.9. Partial correlations (age controlled) among numeracy tasks.

Mexican sample above the diagonal, Canadian sample below the diagonal.

\begin{tabular}{|c|c|c|c|c|c|c|c|}
\hline & Verbal count & Digit quantity & Word quantity & Word digit & Give $\mathrm{N}$ & KeyMath & $\begin{array}{l}\text { Verbal } \\
\text { Comp }\end{array}$ \\
\hline Verbal count & -- & $.55 * * *$ & $.43 * * *$ & $.43 * * *$ & $.63 * * *$ & $.67 * * *$ & $.41 * *$ \\
\hline Digit-quantity & .23 & -- & $.48 * * *$ & $.62 * * *$ & $.53 * * *$ & $.65 * * *$ & $.39 * *$ \\
\hline Word-quantity & $.35^{*}$ & $.73 * * *$ & -- & $.62 * * *$ & $.55 * * *$ & $.62 * * *$ & $.62 * * *$ \\
\hline Word-digit & $.32 *$ & $.59 * * *$ & $.58 * * *$ & -- & $.50 * * *$ & $.65 * * *$ & $.54 * * *$ \\
\hline Give N & .28 & $.42 * *$ & $.57 * * *$ & $.45^{* *}$ & -- & $.70 * * *$ & $.47 * * *$ \\
\hline KeyMath & $.47 * *$ & $.56^{* * *}$ & $.68 * * *$ & $.47 * * *$ & $.60 * * *$ & -- & $.58 * * *$ \\
\hline Verbal Comp. & $.41 * *$ & $.64 * * *$ & $.68^{* * *}$ & $.46^{* *}$ & $.43 * *$ & $.54 * * *$ & -- \\
\hline
\end{tabular}

${ }^{*} p<.05, * * p<.01, * * * p<.001$. 


\section{Path analyses}

The hypothesized model of relations among numeracy measures is presented in Figure 4.6. The hypothesized model was based on the results of Study 1. There were however two differences, first, as noted in the introduction, a more direct measure of cardinality was used as opposed to the quantity knowledge tasks used in Study 1; second, symbol identification skills were not assessed in the present study. As shown in Figure 4.6, children's knowledge of the verbal counting sequence was expected to predict their knowledge of cardinality. Knowledge of the cardinality principle was expected to predict children's ability to map number words to quantities and number words to digits. Both these mapping tasks were expected to predict mapping between digits and quantities, which was expected to predict children's verbal comparison skills. The direct path from cardinality to verbal comparison was included because results from Study 1 showed that although there was a marginal relationship between digit-quantity mapping and verbal comparison, the strongest predictor was children's quantity knowledge which tapped into children's understanding of the cardinal principle.

Given the modest sample sizes the path analyses are considered exploratory in nature. Note however that the sample size issue is mitigated by the fact that the model presented in the present research is intended as a replication of the results from Study 1. 
Figure 4.6 Hypothesized model of relations among numeracy tasks based on the results from Study 1.

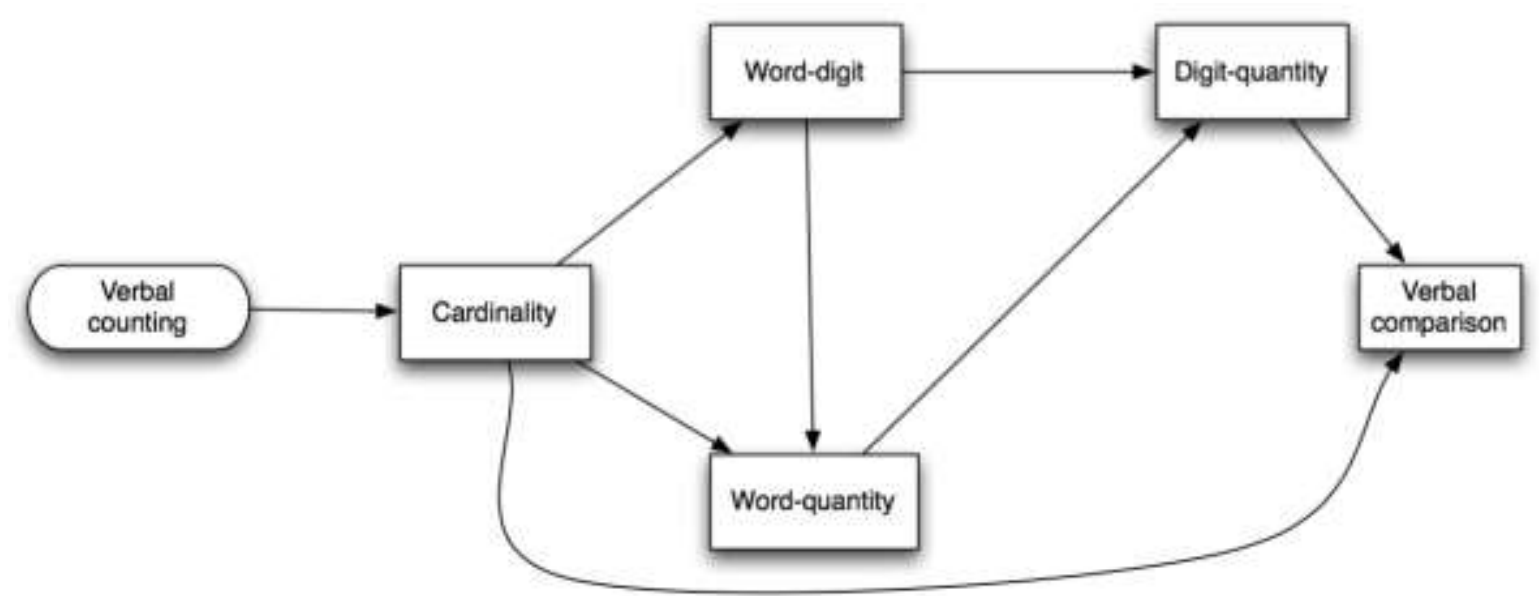

A multi-group path analysis was conducted to assess the hypothesized model. Age was controlled for in all regressions and the relationship between age and each outcome variable was allowed to differ. Pathways shown were based on those identified in Study 1. The analytic approach that I used was to start with a constrained model in which all paths were set to be equal in both samples was assessed. The constrained model had a very poor fit to the data, $\chi^{2}(22)=80.75, p<.001 ; R M S E A=.23,[90 \%$ CI .17 to.28]; $C F I$ $=.84, S R M R=.15$. Subsequently, to improve model fit, modification indices were examined and the path from verbal counting to Give N (cardinality) was allowed to differ between the groups. This modification was considered sensible given the differences in verbal counting skill between the groups and the pattern of partial correlations between performance on the Give $\mathrm{N}$ and verbal counting (see Table 4.9). Allowing the path to differ significantly improved the fit of the model as shown by the reduction in the chi square value, however the overall fit was still poor: $\chi^{2}(21)=56.90, p<.001 ; R M S E A$ $=.18,[90 \%$ CI .127-.241]; CFI $=.90, S R M R=.11$. 
Next, the modification indices suggested that the path from word-quantity to digit quantity be freed to vary between groups. Given Mexican children's poor performance on the symbol-quantity mapping tasks it was possible that they were not relying on their word-quantity mapping to solve the task but more so on their verbal counting skill. Allowing the path between word-quantity and digit-quantity to vary between groups significantly reduced the chi square value, but the model fit was still unacceptable, $\chi^{2}(20)$ $=44.99, p=.001 ; R M S E A=.16,[90 \%$ CI .095-.218]; $C F I=.93, S R M R=.076$. A direct path from verbal counting to digit-quantity (which was also suggested by the modification index) was added for the Mexican sample only, the addition of this path greatly reduced the chi square but the model was still a poor fit, $\chi^{2}(19)=36, p=.01$; RMSEA $=.13,[90 \%$ CI .063-.198]; $C F I=.95, S R M R=.067$. The modification indices further suggested the addition of a path from word-quantity to verbal comparison in both samples. Because it is assumed that children's ability to compare two symbolic number words would be dependent on their symbol-quantity mapping skills, the direct path was added to both models. The addition of the path resulted in a model with excellent fit: $\chi^{2}(18)=14.88, p=.67 ;$ RMSEA $=.00,[90 \%$ CI .000-.101]; CFI $=1.00, S R M R=.05$. Results for this final model are presented in Figure 4.7 and Tables 4.10, 4.11, and 4.12. As shown in Figure 4.7, overall, the differences between the models from each country were related to the relations between verbal counting and the other variables, and the relations among the symbol-quantity mappings. For Mexican children, significant direct and indirect effects of verbal counting on all tasks were found (See Tables 4.10 and 4.12), in contrast, for Canadian children there was only a significant direct effect from verbal counting to Give N (Tables 4.10 and 4.11). 
For Canadian children, both word-digit and word-quantity mapping predicted digit-quantity mapping (as in Study 1) but a different pattern was found for Mexican children where digit-quantity mapping was predicted by word-digit mapping and verbal counting but not by word-quantity mapping. Thus for Mexican children, knowledge of verbal counting and word-digit mapping seemed to be critical to their performance on the more complex digit-quantity mapping, likely because they are still in the process of acquiring these basic skills.

Finally, in both groups, both word-quantity and digit-quantity directly predicted verbal comparison, with significant indirect effects from cardinality and word-digit mapping. The results are thus consistent with findings from Study 1 and provide evidence that understanding symbol-quantity mapping is crucial for children to perform symbolic number comparison. 
Figure 4.7. Significant standardized effects from the multi-group path analyses.

Top figure Canadian sample; bottom figure Mexican sample. Standardized coefficients are shown. Dotted lines are non-significant paths that had been hypothesized in the original model.
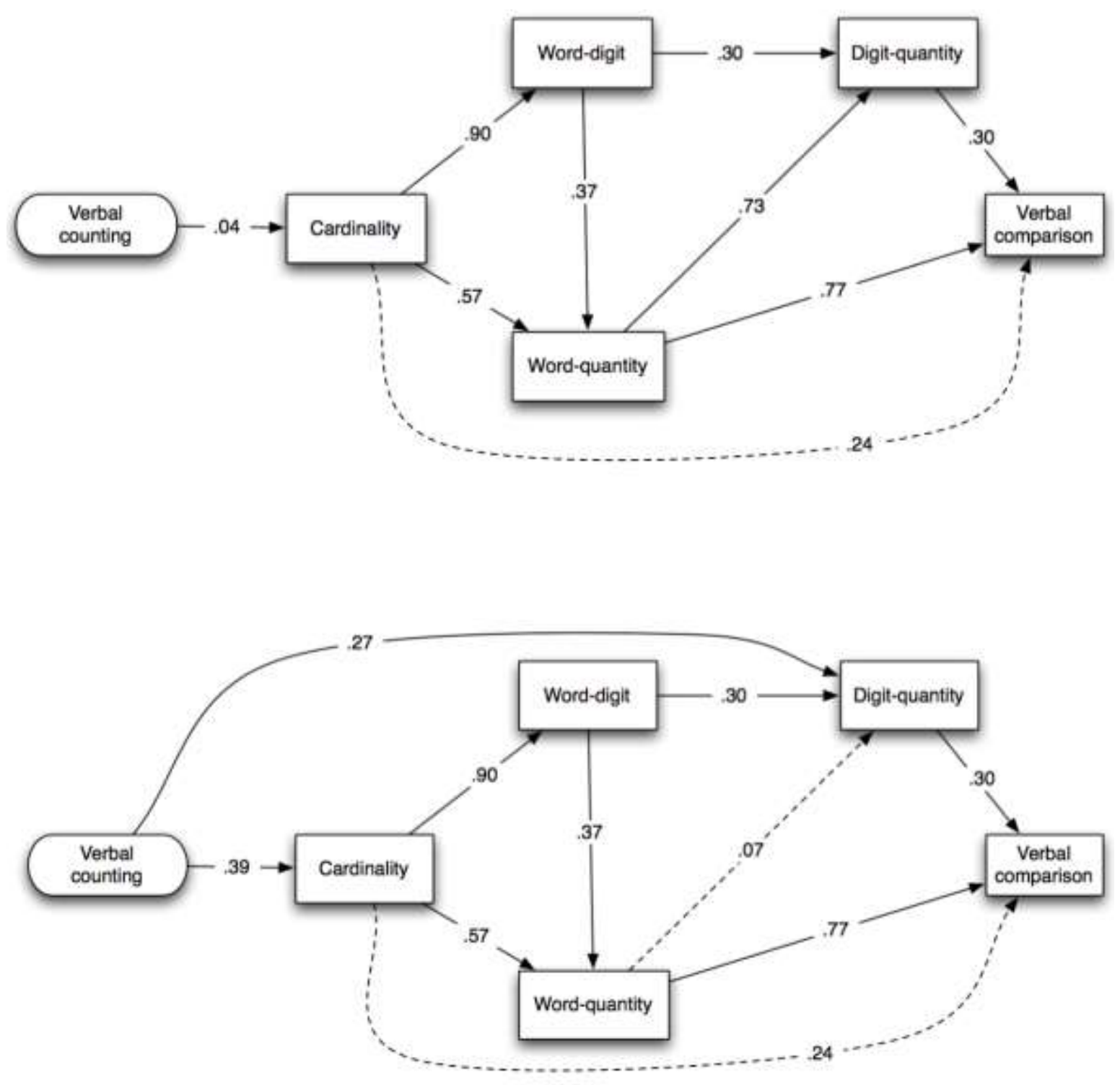
Table 4.10. Path analysis: Unstandardized direct, total indirect and total effects for the multi-group path analyses by country.

\begin{tabular}{|c|c|c|c|c|c|c|c|c|c|}
\hline \multirow[b]{2}{*}{ Outcome } & \multirow[b]{2}{*}{ Predictor } & \multicolumn{4}{|c|}{ Canada $(n=45)$} & \multicolumn{4}{|c|}{ Mexico $(n=57)$} \\
\hline & & Direct & $\begin{array}{c}\text { Total } \\
\text { Indirect }\end{array}$ & $\begin{array}{c}\text { Total } \\
\text { Effect }\end{array}$ & $R^{2}$ & Direct & $\begin{array}{c}\text { Total } \\
\text { Indirect }\end{array}$ & $\begin{array}{c}\text { Total } \\
\text { Effect }\end{array}$ & $R^{2}$ \\
\hline Count & Age & $0.72 * * *$ & & & .17 & $0.41 * * *$ & & & $.24 *$ \\
\hline Give N & $\begin{array}{l}\text { Age } \\
\text { Count }\end{array}$ & $\begin{array}{l}0.12 * * * \\
0.04 *\end{array}$ & & & $.39 * * *$ & $\begin{array}{l}0.01 \\
0.39 * * *\end{array}$ & & & $.47 * * *$ \\
\hline Word-Digit & $\begin{array}{l}\text { Age } \\
\text { Count } \\
\text { Give N }\end{array}$ & $\begin{array}{l}0.07 \\
0.90 * * *\end{array}$ & 0.03 & 0.03 & $.37 * * *$ & $\begin{array}{l}0.14 \\
0.90 * * *\end{array}$ & $0.35 * * *$ & $0.35 * * *$ & $.32 * * *$ \\
\hline Word-quantity & $\begin{array}{l}\text { Age } \\
\text { Count } \\
\text { Give N } \\
\text { Word-digit }\end{array}$ & $\begin{array}{l}0.05 \\
0.57 * * * \\
0.37 * * *\end{array}$ & $\begin{array}{l}0.03 \\
0.33 * * *\end{array}$ & $\begin{array}{l}0.03 \\
0.90 * * *\end{array}$ & $.58 * * *$ & $\begin{array}{l}0.08 \\
0.57 * * * \\
0.37^{* * *}\end{array}$ & $\begin{array}{l}0.35 * * * \\
0.33 * * *\end{array}$ & $\begin{array}{l}0.35 * * * \\
0.90 * * *\end{array}$ & $.55 * * *$ \\
\hline Digit-quantity & $\begin{array}{l}\text { Age } \\
\text { Count } \\
\text { Give N } \\
\text { Word-digit } \\
\text { Word-quantity }\end{array}$ & $\begin{array}{l}0.30 * * * \\
0.73 * * *\end{array}$ & $\begin{array}{l}0.03 \\
0.92 * * * \\
0.27 * * *\end{array}$ & $\begin{array}{l}0.03 \\
0.92 * * * \\
0.57 * * *\end{array}$ & $.67 * * *$ & $\begin{array}{l}0.02 \\
0.27 * * \\
0.30 * * * \\
0.07\end{array}$ & $\begin{array}{l}0.13 * * \\
0.33 * * * \\
0.03\end{array}$ & $\begin{array}{l}0.40 * * * \\
0.33 * * * \\
0.33 * * *\end{array}$ & $.53 * * *$ \\
\hline $\begin{array}{l}\text { Verbal } \\
\text { comparison }\end{array}$ & $\begin{array}{l}\text { Age } \\
\text { Count } \\
\text { Give N } \\
\text { Word-digit } \\
\text { Word-quantity } \\
\text { Digit-quantity }\end{array}$ & $\begin{array}{l}0.24 \\
0.77 * * * \\
0.30 *\end{array}$ & $\begin{array}{l}0.05 \\
0.96^{* * *} \\
0.46^{* * *} \\
0.22^{\dagger}\end{array}$ & $\begin{array}{l}0.05 \\
1.20 * * * \\
0.46^{* * *} \\
0.99 * * *\end{array}$ & $.67 * * *$ & $\begin{array}{l}0.24 \\
0.77 * * * \\
0.30 *\end{array}$ & $\begin{array}{l}0.48 * * * \\
0.79 * * * \\
0.39 * * * \\
0.02\end{array}$ & $\begin{array}{l}0.48 * * * \\
1.03 * * * \\
0.39 * * * \\
0.79 * * *\end{array}$ & $.47 * * *$ \\
\hline
\end{tabular}


Table 4.11. Path analysis: Other significant (unstandardized) indirect effects for the Canadian sample.

\begin{tabular}{llc}
\hline Outcome & Indirect Effects & Estimate \\
\hline Word-quantity & Give N through word-digit & $0.33^{* * *}$ \\
Digit-quantity & Give N through word-quantity & $0.41^{* * *}$ \\
& Give N through word-digit, & $0.27^{* * *}$ \\
& Give N through word-digit and digit-quantity & $0.24^{* * *}$ \\
& Word-digit through word-quantity & $0.27^{* * *}$ \\
Verbal comparison & Give N through word-quantity & $0.44^{* * *}$ \\
& Give N through word-digit and word-quantity & $0.26^{* *}$
\end{tabular}

${ }^{*} p \leq .05, * * p \leq .01$

Table 4.12. Path analysis: Other significant (unstandardized) indirect effects for the Mexican sample.

\begin{tabular}{llc}
\hline Outcome & Indirect Effects & Estimate \\
\hline Word-digit & Count through Give N & $0.35^{* * *}$ \\
Word-quantity & Count through Give N & $0.22^{* * *}$ \\
& Count through Give N, and word-digit & $0.13^{* * *}$ \\
& Give N through word-digit & $0.33^{* * *}$ \\
Digit-quantity & Count through Give N and word-digit & $0.10^{* *}$ \\
& Give N through word-digit & $0.27^{* * *}$ \\
Verbal comparison & Count through Give N and word-quantity & $0.17^{* *}$
\end{tabular}


Count through Give N, word-digit and word-quantity $\quad 0.10 * *$

Give N through word-quantity

Give $\mathrm{N}$ through word-digit and word-quantity

Word-digit through word-quantity

$* p \leq .05, * * p . \leq 01, * * * p \leq .001$

In summary, the pattern of relationships among the numeracy variables in the Mexican children differed from that of the Canadian children in three main aspects. First, verbal counting had a direct effect on Mexican children's performance on the digitquantity mapping. Second, the hypothesized predictive path from word-quantity to digit quantity was only found for the Canadian children. Third, Mexican children's performance on verbal counting had indirect effects on all measures; in contrast, for the Canadian children, verbal counting had no indirect effects on any of the tasks. The encountered differences could reflect Mexican children's poorer counting skill as compared to the Canadian children.

Overall however, for both countries the overall pattern of results was broadly consistent with the hypothesized model. Replicating the results of Study 1, verbal counting predicted children's cardinality knowledge which in turn was related to both the word-digit and the word-quantity mappings. A difference from Study 1 was that in the present study, children's word-quantity and digit-quantity mappings were directly related to their ability to compare two number symbols whereas in Study 1, verbal comparison was found to be marginally related to children's digit-quantity mapping and was predicted by quantity knowledge. However, the results from the present study support the 
hypothesis that children's symbol-quantity mapping is an important predictor of children's symbolic comparison skill.

\section{Order of acquisition of the mappings}

To examine the order in which children acquire the mappings between number representations, children were categorized into those who performed above chance (i.e., obtained a score of 14 or higher on each mapping task, $p<.05$ binomial) and those whose performance did not differ from chance on each of the six mapping tasks. Table 4.13 shows the percentage of children who performed above chance on each mapping task and the percentage of children who performed above chance on both tasks for each mapping pathway in both countries. Replicating the results of Study 1, in both countries, a higher percentage of children performed above chance on the number-word-digit mapping tasks and a lower percentage of children performed above chance on the digit-quantity mapping tasks.

Contingency tables for children who performed at and above chance on worddigit, word quantity, and quantity-digit mappings are presented in Tables 4.14 and 4.15. Categorical and logistic regression analyses were used to assess the probability that children would succeed on the digit-quantity mapping based on whether they succeeded (or not) on the word-digit and word-quantity mappings. In both countries, the pattern of results was similar. For example, all the children (in Canada and Mexico) who failed to perform above chance on digit-word mapping did not perform above chance on digitquantity mapping Given the complete separation in both the Mexican and Canadian children, logistic regression analyses predicting digit-quantity mapping from word-digit mapping were not performed. 
Logistic regression analyses showed that word-quantity mapping significantly predicted digit-quantity mapping in both countries. For Mexican children: $B=3.09, S E=$ 1.18, Wald $=6.84, p=.009$, Odds Ratio $=22.00,95 \%$ CI $[2.17-223.23]$. For Canadian children: $B=2.64, S E=0.86$, Wald $=9.37, p=.002$, Odds Ratio $=14.06,95 \%$ CI $[2.59$ 76.35]. In both countries, children who failed to perform above chance on the wordquantity mapping had less than $9 \%$ probability of succeeding on the digit-quantity task. Thus, consistent with Study 1, data from children in both countries provides evidence that children acquire the digit-quantity mapping once they are able to map number words to digits and number words to quantities.

As in Study 1, I hypothesized that word-digit mapping would develop prior to word-quantity mapping in both countries, the contingency tables for both Mexican and Canadian children and logistic regression analyses are presented in Appendix J. For the Mexican children, those who successfully mapped digits to number words were significantly more likely to perform above chance on the word-quantity mapping task. Replicating the results from Study 1, none of the Canadian children who performed at chance on word-digit mapping were successful on word-quantity mapping. 
Table 4.13. Percentage of children who performed above chance on each of the three sets of mapping tasks in both countries.

The combined column indicates the percentage of children who performed above chance on both orders for that pair of tasks.

\begin{tabular}{|c|c|c|c|c|c|c|}
\hline \multirow[b]{3}{*}{ Mapping Tasks } & \multicolumn{3}{|c|}{ Mexico } & \multicolumn{3}{|c|}{ Canada } \\
\hline & \multicolumn{2}{|c|}{ Direction } & \multirow[t]{2}{*}{ Combined } & \multicolumn{2}{|c|}{ Direction } & \multirow[t]{2}{*}{ Combined } \\
\hline & 1 & 2 & & 1 & 2 & \\
\hline Number Words, Digits & 39 & 40 & 35 & 84 & 89 & 80 \\
\hline Quantities, Number words & 33 & 28 & 21 & 62 & 60 & 44 \\
\hline Quantities, Digits & 9 & 19 & 9 & 47 & 40 & 29 \\
\hline
\end{tabular}

Note. Direction 1 corresponds to the order of the tasks in the Mapping Tasks column. 
Table 4.14. Contingency table for word-digit, digit-quantity mapping tasks in both countries.

\begin{tabular}{lccrc}
\hline & \multicolumn{3}{c}{ Mexico } & Canada \\
\cline { 2 - 5 } & & & \multicolumn{2}{c}{ Digit-quantity } \\
\hline Word-digit & Chance & Above Chance & Chance & Above Chance \\
\hline Chance & & 0 & 9 & 0 \\
Above Chance & 37 & 5 & 23 & 13 \\
\hline
\end{tabular}

Table 4.15. Contingency table for word-quantity, digit-quantity mapping tasks in both countries.

\begin{tabular}{lcccc}
\hline & \multicolumn{3}{c}{ Mexico } & Canada \\
\cline { 2 - 5 } & & & Digit-quantity \\
\hline Word-quantity & Chance & Above Chance & Chance & Above Chance \\
\hline Chance & & 44 & 23 & 9 \\
Above Chance & 8 & 4 & 2 \\
\hline
\end{tabular}




\section{Summary}

The present study had two main goals: a) to compare Mexican and Canadian children's performance on early numeracy tasks, and b) to examine whether the pattern of relationships among children's early numeracy skills would be similar among children in both countries.

Children were assessed on tasks that included a) knowledge of the verbal counting string and cardinality, b) their ability to map among the three representations of number, c) their ability to perform symbolic verbal comparison, and d) their early numeracy knowledge. As children in Mexico had been enrolled in formal school for seven months at the time of testing, I had expected their performance to be higher than that of Canadian children who were still in daycare; however, this hypothesis was not supported. Mexican children performed significantly worse on all measures than did Canadian children. This overall difference in early numeracy skill may limit the validity of the comparison of the patterns of relations across groups.

Results showed that Canadian children had good counting and cardinality skills; Mexican children on the other hand could not, on average, count beyond 9 and the majority were still developing their cardinality knowledge. Canadian children showed above chance performance on all mapping tasks at the small, medium, and large set sizes whereas the Mexican children performed above chance on all three set sizes only on the word-digit mapping. Nevertheless, despite the mean differences in performance between the two groups, the encountered patterns of performance supported findings from Study 1 and prior proposals by Bialystok (1992): In both countries, performance on the word- 
digit mapping task was higher than on the symbol-quantity mapping tasks and lowest on the digit-quantity mapping.

According to prior research, symbolic comparison tasks require an underlying knowledge of the quantities that are represented by the number symbols (e.g., Batchelor, Inglis, \& Gilmore, 2015). In the present study, Canadian children performed above chance on the verbal comparison tasks for both distance 1 and distance 3; Mexican children's performance on the other hand did not differ from chance. This pattern of results was not surprising given Canadian children's significantly better performance on the cardinality and mapping tasks. The results are consistent with research finding that children with who are unable to map the symbolic and non-symbolic representations of quantity show lower performance on more complex math tasks such as symbolic comparison tasks (e.g., Rouselle \& Noël, 2007).

In the present study, the model in Study 1 was tested. The findings from the Canadian sample were overall consistent with the initial model from Study 1 with one exception. In the present study, cardinality knowledge was not a significant predictor of children's verbal comparison skill; in contrast, it was children's word-quantity and digit quantity mappings that predicted children's ability in comparing two verbal number words. This difference across studies may reflect differences in the tasks: in Study 1 the tasks involved reproducing sets of objects without the use of number words; in contrast in Study 2 the task involved asking the child to produce a set of 1-6 objects. To succeed on the set reproduction task (Study 1) children had to capture a quantity and reproduce it and so solving the task probably involved a process of counting the objects and keeping the number to be reproduced in working memory. In contrast, in the Give N task (Study 2) 
children were told the number of objects they needed to provide, furthermore, the children were asked to count the objects to make sure that the amount they provided was correct. Thus the working memory demands of the set reproduction task (particularly for the hidden version) were greater than those of the Give $\mathrm{N}$ task. When performing verbal number comparison, children need to keep two numbers in working memory in order to compare them; thus the direct relation found in Study 1 could be due to the working memory component of the task. Despite this difference, the pattern of relationships for the rest of the measures was replicated and the hypothesis that children need to master the symbol-quantity mappings in order to perform more complex math tasks was again supported by the present data.

The overall pattern of relationships proposed in Study 1 among the early numeracy measures had not been expected to differ between the two countries. This hypothesis was partially supported. Results from the path analyses showed two main differences between the final models from the Mexican and Canadian samples. First, for the Mexican children, there were significant indirect effects of verbal counting on all the outcomes. Second, in contrast to the Canadian children whose performance on both word-digit and word-quantity tasks predicted their ability to map digits to quantities, for the Mexican children, digit-quantity mapping was not related to children's word-quantity mapping and was instead predicted by verbal counting and children's word-digit mapping.

What factors could account for the differences in the patterns of performance between Canadian and Mexican children? Prior research has found that the home learning environment (e.g., practices, academic expectations) is predictive of children's numeracy 
performance (Blevins-Knabe et al., 1996; Kleemans et al., 2012; LeFevre et al., 2002;

LeFevre et al., 2009; LeFevre, Polyzoi et al., 2010; Manolitsis et al., 2013; Skwarchuk, 2009). Research has found that parental home practices and expectations tend to differ as a function of cultural background (e.g., Reese \& Gallimore, 2000; Huntsinger et al., 2000; LeFevre, Polyzoi et al., 2010); socio-economic status (e.g., DeFlorio \& Beliakoff, 2015; Starkey \& Klein, 2008), and parent education (e.g., Harding et al., 2015). Thus, to the extent that the home experiences for the two groups of children are different, home numeracy practices may, in part, account for the differences we observed across groups.

Researchers have found differences between North American and Latino parental beliefs concerning their children's ability to learn prior to entering school. For example, North American parents engage their children in early literacy activities such as story book reading long before their children are enrolled in school, whereas low SES Latino parents believe that prior to entering school, children are unable to benefit from such experiences; as a result, children are less likely to be engaged in early learning activities prior to entering school (Reese \& Gallimore, 2000). In the present study, children in Mexico were recruited from schools serving low- to middle-SES families. Research on differences in home learning environment and SES has shown that low-SES parents tend to have less accurate beliefs about the skills that their children can develop (e.g., DeFlorio \& Beliakoff, 2015). Hence, parents may not provide beneficial early numeracy activities because of a lack of knowledge about children's numeracy development. Finally, as shown in Table 4.1, the level of education for parents was significantly lower in the Mexican than in the Canadian group. Maternal education has been found to be related to children's academic performance (Harding et al., 2015; Lareau, 2011) and 
specifically their math learning (e.g., Gonzalez Estudillo, 2011). Highly-educated mothers provide more stimulating activities and conversations; they are more likely to seek better opportunities for their children both in and out of school; and children may benefit from role models that value education (Harding et al., 2015). All of these factors may have contributed to the differences between the two groups.

Another factor that should be examined is the education children are exposed to in preschools and daycares. In Canada, teachers are not expected to engage in formally teaching the children academic skills; children in daycare are exposed to a play-based environment. Preschool teachers in Mexico are expected to directly teach the children math and literacy, and thus children presumably engage in formal symbolic numeracy practices such as writing numbers in their notebooks or worksheets more frequently. Notably, however, research has shown that children benefit most from preschool or daycare activities when they receive at least a modest amount of support at home (e.g. Anders et al., 2012).

The results from Study 2 were consistent with the model proposed in Study 1. Canadian and Mexican children's knowledge of quantity and cardinality were found to be predictive of children's word-quantity mapping which in turn is predictive of children's digit-quantity mapping. In the next chapter I explore whether enhancing children's cardinality skills would result in an improvement on children's digit-quantity mapping skills. 


\section{CHAPTER 5: STUDY 3}

\section{Introduction}

Study 1 provided evidence that children's understanding of how number words are used to represent quantity is an important predictor of their symbol-quantity mapping skills. The model assessed in Study 1 showed that children's ability to mentally represent and manipulate quantities was found to have direct and indirect effects on their ability to map number words to quantities as well as indirect effects on the digit-to-quantity mapping through both number-word-digit and number-word-quantity mapping. In order to successfully map number symbols to quantities, children need to learn the meaning of the symbol (even when the physical set is absent). In order to gain this understanding, children must acquire the ability to determine either through subitizing (for the smaller numbers) or counting (for the larger numbers the amount of objects in a set); that is, children must understand be able to understand that the last word in a count represents the total number of objects in a set (i.e., cardinality). Thus, I hypothesized that an intervention that targeted children's knowledge of the cardinal principle would result in an improvement of children's digit-quantity mapping skills (see Figure 5.1; Right panel $)^{11}$. The present study was designed to provide further assessment of the model in Study 1 by testing the hypothesis that children's precursor cardinality skill is important for the development of the symbol-quantity mappings.

\footnotetext{
${ }^{11}$ For the group of children who participated in the intervention that targeted cardinality, their digit-quantity mapping was hypothesized to show improvement only to the extent to which children had developed the mapping between number words and digits. For example, if a child could not map the digits to the number words beyond 7, training on cardinality would not be expected to improve digit-quantity mapping beyond 7 .
} 
To provide a comparison condition, the present study included a second training condition that targeted the mapping between number words and digits. The training that was designed to enhance the link between number words and digits was hypothesized to improve only children's word-digit mapping skills (Figure 5.1; Left panel); children's symbol-quantity mapping skills were not expected to show an improvement because the word-digit training would not provide children with practice associating symbolic number representations to the corresponding quantities.

Previous research has shown that interventions designed to enhance children's cardinality (Mix, Sandhofer, Moore, \& Russel, 2012), counting, and number naming skills (Dyson, Jordan, \& Glutting, 2011; Jordan, Glutting, Dyson, Hassinger-Das, \& Irwin, 2012; Krohn, Skinner, \& Fuller, 2012) significantly improve these early numeracy skills. However, the issue of whether enhancing children's cardinality knowledge and their ability to associate number words to digits might also influence their ability to associate digits to their corresponding quantity representations has not yet been examined. The present study used a pre-test, intervention, post-test design to address the issue of whether improving children's cardinality skills would result in an improvement in their digit-quantity mapping abilities.

The training procedures in the present Study were designed following Mix et al. (2012). Mix and colleagues designed five training conditions in which they used a booklet containing 36 pictures of sets of 1-9 objects with three-and-a-half-year-oldchildren. Children participated in one of five training procedures: a) labeling and counting the sets, b) counting the set without verbally labeling it first, c) only verbally labeling the set but not counting it, d) alternating counting and labeling across the 
different sets, and e) a control condition in which the child and the experimenter talked about the objects in the booklet without either counting or labeling the quantities. Mix et al. found that children who participated in the labeling and counting training condition (d) showed significant improvement on their cardinality skill after the six training sessions ${ }^{12}$.

Following Mix et al. (2012), in the present study, training materials for the cardinality condition included cards containing colorful sets of 1-9 objects and, as in Mix et al., participating children were engaged in activities that involved verbally labeling and counting sets of objects. There were however, three main differences between the procedure used in Mix et al. and the one used in the present study. First, children in the study by Mix et al. were trained individually while those in present study were trained with a partner. The decision to train children in pairs was made in order to encourage participation as some children feel more comfortable "playing" with a partner; and to maximize the use of the available time (e.g., some daycares allowed only a limited amount of time for the study). Second, the intervention lasted two sessions as opposed to the six training sessions in Mix et al. Third, in the present study, children in both the cardinality and the symbolic conditions participated in three different activities in each of the two sessions as opposed to a single activity used by Mix and colleagues. The different activities were used in order to maximize the possible learning by presenting the children with the opportunity to both listen to the correct verbal label for the quantity and to produce the verbal labels themselves.

Figure 5.1 Predicted patterns of improvement by training conditions.

\footnotetext{
12 Specifically, the counting and labeling training procedure consisted of 1) the experimenter verbally labeling the set, 2) the experimenter counting the set, and 3) children labeling and counting the sets.
} 
Digit to word mapping is shown in the left panel; digit-quantity mapping in the right panel

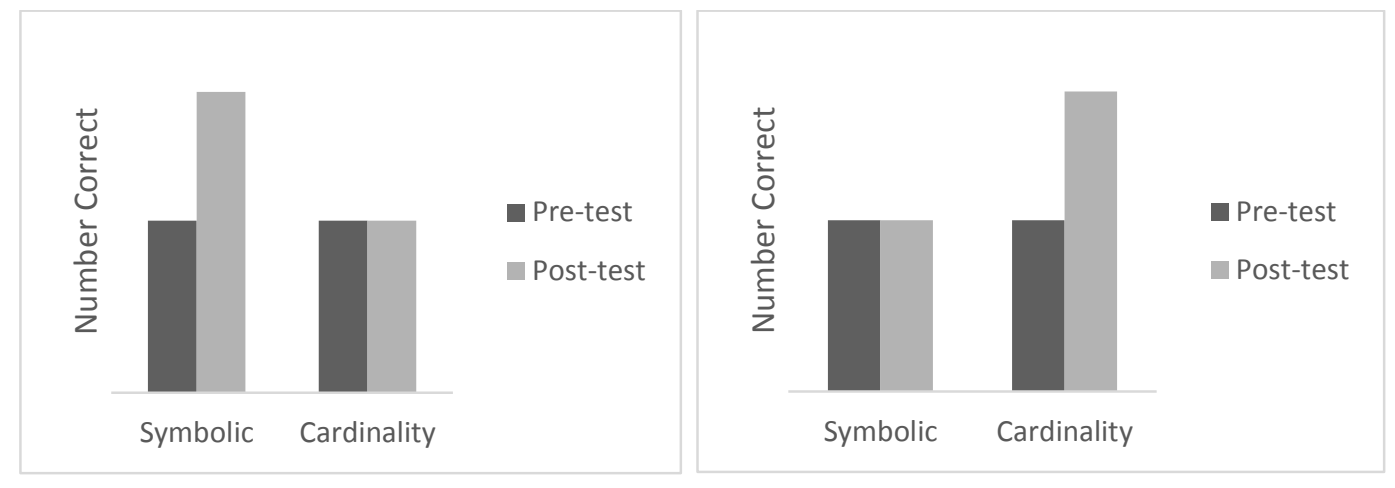

\section{Method}

\section{Participants}

A subset of forty Canadian children from Study $2^{13}$ (19 girls), were randomly assigned to one of two training conditions designed to enhance either a) their numberword-to-digit mapping (symbolic training) or b) their cardinality knowledge (cardinality training).

Mean age of the children was 48.10 months $(S D=5.82$, range $=36-60$ months $)$. The frequency distribution for children's age is presented in Appendix K. Seventeen children spoke another language in addition to English: French (7), Chinese (12), and Spanish (2); one child spoke French and Vietnamese. Information on parental education was available for 39 mothers and 35 fathers. Parents of participating children were highly educated; $61 \%$ of the mothers had post graduate degrees, $31 \%$ had a university degree, $5 \%$ had a college degree, and 3\% had a high school diploma. For the fathers, $57 \%$ had post graduate degrees, $26 \%$ had university degrees, $14 \%$ had a college degree, and $3 \%$ had a high school diploma.

${ }^{13}$ One child moved away, two children refused to participate and 2 children did not complete training due to time restrictions in their daycare. 
There were a total of 20 children per training group. There was no difference in age between the two groups: cardinality training: $M=47.10, S D=6.73$; Symbolic training: $M=49.00, S D=4.72, t(38)=1.03, p=.31$. The distribution of boys and girls did not differ between training groups, $\chi^{2}(1, N=40)=0.90, p=.34$, Cramer's $V=.15$. The distribution of children who spoke a different language in addition to English at home did not differ across training groups, $\chi^{2}(1, N=40)=0.40, p=.52$, Cramer's $V=$ .10 .

\section{Procedure}

Children completed pre-testing during their participation in Study 2. For the Study 3 intervention, children were randomly assigned to one of two training groups: a) symbolic training, and b) cardinality training. For both intervention groups, children were assigned a partner with whom they played three number card games on each of two 20minute sessions that were conducted no more than one week apart.

\section{Cardinality Training}

For the first two games which involved naming all the quantities 1-9, a "deck" of 9 laminated white $(15 \times 10 \mathrm{~cm})$ cards with 1-9 figures printed on them were used (see Figure 5.2 top panel for examples). Each time a game was played, a single type of figure was used. There were six different types of figures (i.e., fish, balloons, stars, monkeys, frogs and apples) so that the children could choose the figure they wished to play with every time. Thus, for each type of figure a complete "deck" of 9 cards was available.

For the third game, which involved children finding the quantities, the materials included 9 pairs of cards with a specific type of colorful figure (e.g., frogs, balloons, apples, fish, monkeys, or stars) printed on them (see Figure 5.2 bottom panel for examples). A single type of figure was used every time the game was played; thus, the 
material used each time the game was played was a "deck" of nine pairs of cards with the same type of object printed on them (See figure 5.2 bottom panel). As shown in the bottom panel of figure 5.2, the pairs of cards were bound together by a small metal ring placed on the upper left hand corner. The pairs of cards included number distances of 2 and 4 , and the larger distances were always presented to the children first.

Figure 5.2. Examples the cards used for the cardinality training.

Three complete "decks" of 9 cards (top panel) and three examples of pairs of cards from three different “decks" (bottom panel).
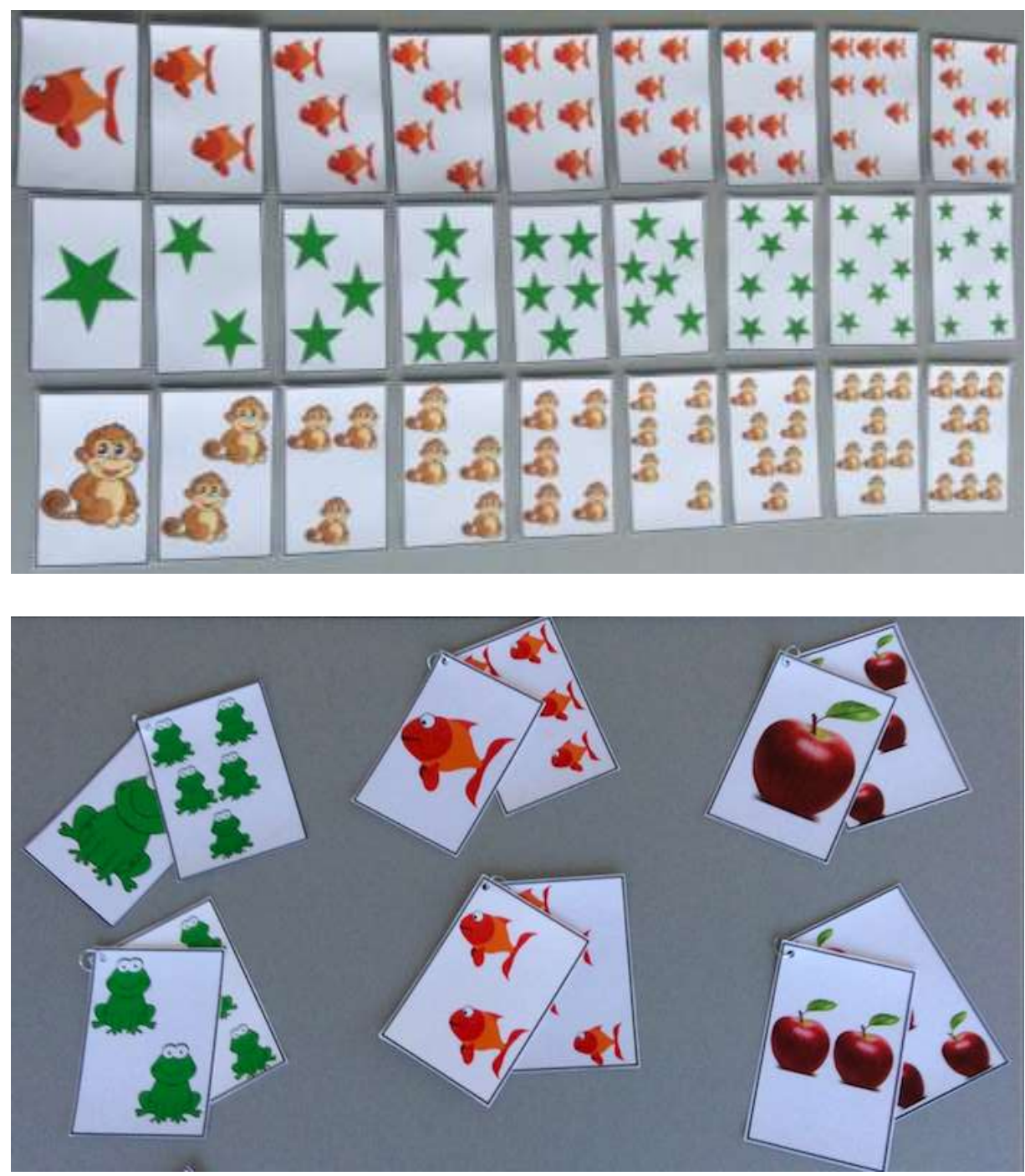
Card games. Following Mix et al. (2012), the cardinality training was designed to enhance children's knowledge of the cardinal principle. Children in this condition played three card games in which they were presented with cards depicting a set of 1-9 figures ${ }^{14}$. For each training game, they: a) listened to the verbal label of a set, b) counted the set, and c) produced the number word corresponding to the cardinality of the set. In each of the three games, children were exposed to all the quantities 1-9 once; thus, during each training session children counted sets of 1-9 three times. Children were verbally encouraged and corrective feedback was provided at all times.

Name the quantities (experimenter). A complete "deck" of nine cards, each of which had 1-9 objects of the same type printed on them (as shown in Figure 5.2 top panel) were used for this game. The experimenter placed a card on the table and asked the children how many objects were on the card, once the children had said a number the experimenter asked each child to count the figures on the card, finally, children were asked once again how many objects there were on the card. The procedure was repeated for the each card, presented in random order.

Name the quantities (children). A complete "deck" of nine cards each of which had 1-9 objects of the same type printed on them (as shown in Figure 5.2 top panel) were used for this game. The experimenter gave one child five of the cards and asked him or her to place them one by one on the table. After the first child placed a card on the table,

\footnotetext{
${ }^{14}$ On the cards that were used for the cardinality training games, the size of the objects was negatively correlated with number. Although children could have potentially associated the numbers with the relative sizes of the objects (e.g., larger numbers are associated with smaller objects) this was unlikely the case as the training emphasized counting the procedure and knowledge of the total amount of objects in the set. The relative size of the objects was never discussed with the children during training nor were children asked to make comparisons (e.g., which card has more or less objects).
} 
the experimenter would ask the other child how many objects there were, then, both children were asked to count the figures. Finally, the experimenter asked both children how many objects there were. The experimenter would then give the remaining four cards to the other child and repeat the procedure.

Find the quantities. For this game there were two identical "decks" of nine pairs of cards for each child. Both decks had the same figures printed on the cards and included pairs of cards with distances of 2 (i.e., 1-3, 2-4, 6-8, and 7-9), and 4 (i.e., 1-5, 2-6, 3-7, 48, 5-9) (See Figure 5.2 bottom panel for examples of the pairs of cards). The two "decks" of cards were always in control of the experimenter and children received the pairs of cards one by one. For example, the experimenter would give each child the pair of cards with 1 and 3 frogs and ask them to find the card with 3 frogs. Once both children had found the card with the three frogs, the experimenter asked the children to show it to her and children were then asked to: a) say how many objects were on the card, b) count them to make sure, and c) were once again asked how many there were; the process was repeated for the other card in the pair (i.e., for this example, the card with 1 frog). Once the children had counted the frogs on both cards, the experimenter would ask the children to give the cards back to her and would repeat the procedure with the next pair. This game was presented in two parts: the experimenter would present the children with the large distance pairs first, and then continue with another game before returning to the small distance pairs.

\section{Symbolic training}

Materials for the two naming games included a single "deck" of 9 white laminated cards $(15 \times 10 \mathrm{~cm})$ with a green, blue, red or purple digit (1-9; font: Times New Roman, 
size: 350 ) printed on each one (see Figure 5.3 right panel). Materials for the third game, find the number, included two identical "decks" of nine pairs of cards (one for each child). As shown in Figure 5.3 (right panel) the two cards were bound together by a small metal ring placed on the upper left hand corner. The pairs of cards included number distances of 2 (i.e., 1-3, 2-4, 6-8, and 7-9), and 4 (i.e., 1-5, 2-6, 3-7, 4-8, 5-9). The larger distances were always presented to the children first. Children were verbally encouraged and given corrective feedback at all times.

Figure 5.3. Examples of the cards used for the symbolic training condition: a complete deck of 9 cards (left panel) and four examples of the pairs of cards (right panel).
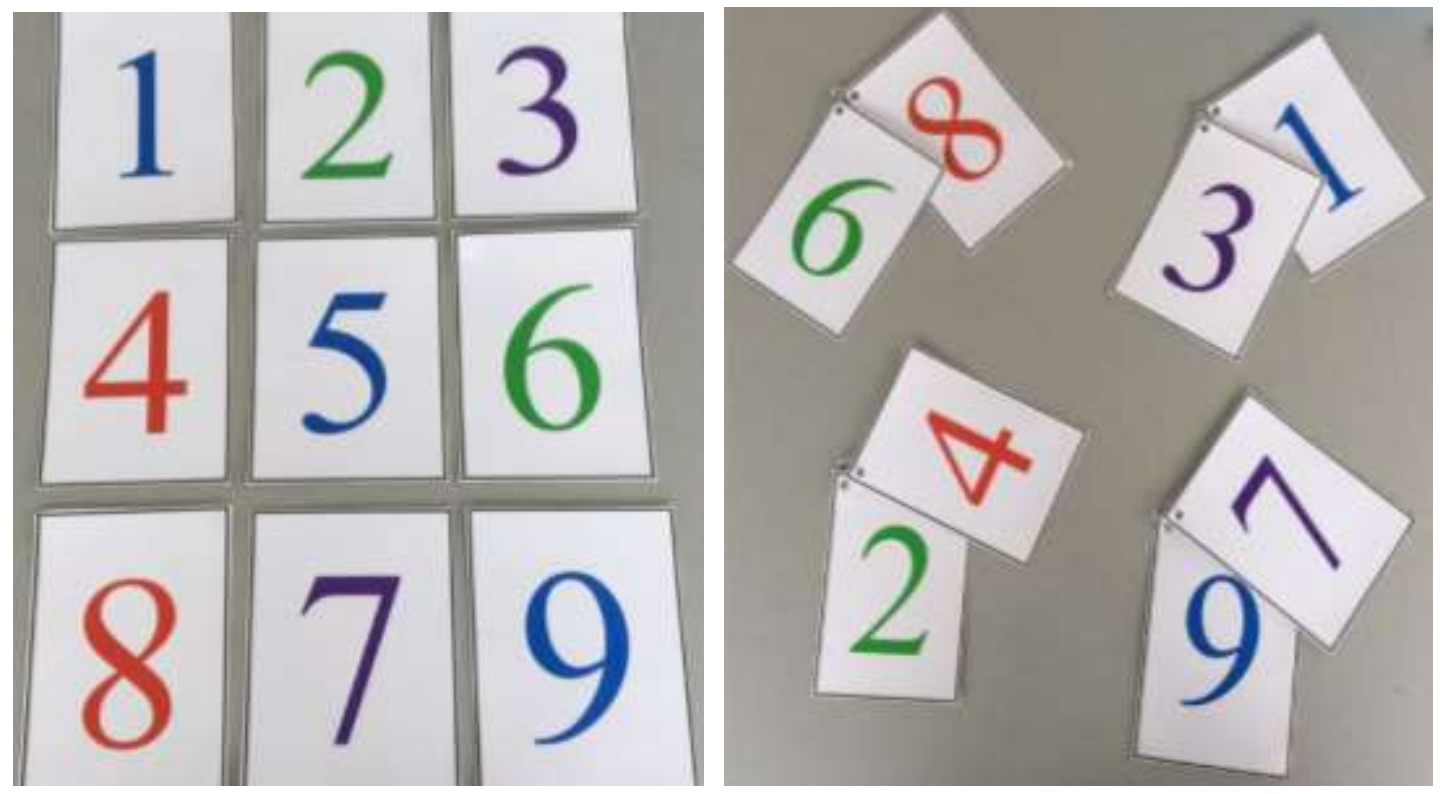

Card games. The purpose of the card games in the symbolic training was to help children learn the mapping between number words and digits. In order to provide the children in both the cardinality and symbolic conditions with similar amount of exposure to the activities, the procedure followed in the symbolic training condition closely resembled that of the cardinality condition. Children in the symbolic training condition played three card games in which they were presented with cards depicting an Arabic 
digit (1-9) and then a) listened to the verbal label of the digit; b) identified the corresponding Arabic digit, and c) produced the number word corresponding to the digit shown in the card. Participants in the symbolic condition were presented with all the digits 1-9 in each of the three games.

Name the digits (experimenter). The experimenter placed a single card (Figure 5.3 left panel) on the table and asked the children to say the number that was written on the card. If one of the children made a mistake, the experimenter would ask them again and corrected them when necessary. The procedure was repeated for all the numbers 1-9.

Name the digits (children). The experimenter gave one child five of the cards (Figure 5.3 left panel) and would ask him or her to place them on the table while the other child named the number. Then the experimenter would then give the remaining four cards to the other child and the first child would name them.

Find the digits. The two identical decks of card pairs (Figure 5.3 right panel) that were used in this game were always in control of the experimenter. The experimenter gave each child the same pair of cards and asked them to find the card with the number $\mathrm{X}$ on it. Once both children had found the number, and showed it to the experimenter, they were asked to find the other number on the pair of cards. This game was presented in two parts: the experimenter would present the children with the large distance pairs first, and then continue with another game before returning to the small distance pairs.

\section{Results}

Means, standard deviations, skew, and kurtosis for both training groups performance on the numeracy tasks at pre- and post-test are presented in Table 5.2. For both groups, the distributions for children's highest count were negatively skewed at pre- 
test. In the symbolic condition, skew was due to two children who counted to $99(z=$ $2.70)$ and $100(z=2.73)$. In the cardinality group the highest count was $31(z=1.98)$. The distributions for both groups on the word-digit, digit-word, and Give $\mathrm{N}$ tasks at pre-test were also non-normal. Transformations did not normalize the distributions. Thus the difference in performance between the groups for these tasks was analyzed with MannWhitney tests. There were no significant differences in performance between the two groups, all $p \mathrm{~s}>.05$.

Differences in performance at pre-test for the word-quantity, quantity-word, digitquantity, quantity-digit, and the standardized scores from the KeyMath Numeration Subtest (because different versions of the test were used at both pre- and post-test) were examined using one-way ANOVAS. The only significant difference was on the KeyMath where children in the Symbolic training condition performed better than children in the Cardinality condition, $F(1,38)=5.98, M S E=0.92, p=.019, \eta_{p}{ }^{2}=.14$.

The verbal comparison task included a total of 20 trials, 10 with a distance of 1 and 10 with a distance of 3 . Differences in performance between training groups on the verbal comparison task at pre-test were analyzed with a 2 (distance: 1 vs. 3 ) x 2(training condition: cardinality vs. symbolic) mixed ANOVA. Across groups, performance on the trials with a distance of 3 was marginally higher $(M=7.35)$ than on the trials with a distance of $1(M=6.80), F(1,38)=3.80, M S E=1.59, p=.059, \eta^{2}{ }_{p}=.09$. Across distances, children in the symbolic training condition performed marginally better $(M=$ 7.80) than children in the cardinality training condition $(M=6.35), F(1,38)=3.68, M S E$ $=11.43, p=.063, \eta_{p}^{2}=.09$. There was no distance by training condition interaction, $F(1$, $38)=0.28, M S E=1.59, p=.60, \eta_{p}^{2}=.01$. 
In summary, children in both groups showed good counting and cardinality skills. Performance at pre-test differed significantly between the two training groups only on the KeyMath with children in the symbolic training condition obtaining higher scores. I next present the results of children's performance at post-test. 
Table 5.1. Descriptive statistics for all measures on pre-test and post-test in Study 3.

\begin{tabular}{|c|c|c|c|c|c|c|c|c|c|c|c|c|c|c|c|c|}
\hline & \multicolumn{16}{|c|}{ Training Condition } \\
\hline & \multicolumn{8}{|c|}{ Symbolic } & \multicolumn{8}{|c|}{ Cardinality } \\
\hline & \multicolumn{4}{|c|}{ Pre-test } & \multicolumn{4}{|c|}{ Post-test } & \multicolumn{4}{|c|}{ Pre-test } & \multicolumn{4}{|c|}{ Post-test } \\
\hline & $M$ & $S D$ & Skew & Kurtosis & $M$ & $S D$ & Skew & Kurtosis & $M$ & $S D$ & Skew & Kurtosis & $M$ & $S D$ & Skew & Kurtosis \\
\hline High Count & 25.70 & 27.16 & $2.20^{*}$ & 4.47 & 33.95 & 34.48 & $1.49^{*}$ & 0.47 & 14.90 & 8.12 & $1.07 *$ & -0.34 & 19.30 & 8.92 & 0.68 & -0.75 \\
\hline Digit-Qty & 13.15 & 3.65 & -0.63 & -0.29 & 13.85 & 2.78 & $-1.53^{*}$ & $2.36^{*}$ & 11.80 & 3.71 & -0.00 & -0.77 & 12.90 & 3.04 & -0.53 & -0.72 \\
\hline Qty-digit & 12.90 & 3.24 & -0.25 & -1.10 & 13.00 & 3.49 & -0.42 & -0.48 & 12.65 & 3.08 & -0.40 & -1.17 & 12.80 & 3.30 & 0.40 & -1.23 \\
\hline Word-Qty & 14.50 & 2.54 & -0.92 & 0.78 & 14.40 & 2.68 & -0.48 & 0.10 & 13.20 & 2.75 & -0.76 & 0.18 & 13.25 & 2.29 & -0.20 & -0.90 \\
\hline Qty-word & 14.60 & 2.06 & -0.43 & 0.38 & 14.65 & 2.72 & -0.62 & 0.19 & 13.10 & 3.31 & 0.02 & -1.61 & 13.90 & 3.41 & -0.71 & -0.73 \\
\hline Digit-word & 16.35 & 2.23 & $-2.13 *$ & $5.41 *$ & 16.40 & 2.19 & $-1.45^{*}$ & 1.05 & 15.25 & 3.84 & $-2.12 *$ & $3.98 *$ & 15.25 & 2.55 & -0.77 & -0.66 \\
\hline \multirow{3}{*}{ Give $\mathrm{N}$} & & & & & & & & & & & & & & \multicolumn{3}{|c|}{$1.70^{*}$} \\
\hline & 5.40 & 1.31 & $-2.08 *$ & $3.18^{*}$ & 5.60 & 0.94 & $-2.43 *$ & 4.97 & 5.05 & 1.39 & -1.00 & -0.60 & 5.55 & 0.89 & - & $2.83 *$ \\
\hline & & & & & & & & & & & & & & \multicolumn{3}{|c|}{$1.92 *$} \\
\hline KeyMath & 4.50 & 1.73 & -0.64 & 0.19 & 4.65 & 1.66 & -0.32 & 0.02 & 3.10 & 1.83 & 0.00 & -1.31 & 4.00 & 1.41 & -0.94 & 0.10 \\
\hline Verbal & 15.65 & 4.09 & $-1.04 *$ & 0.18 & 15.50 & 4.49 & -0.72 & -0.39 & 12.70 & 5.38 & -0.17 & -1.35 & 13.40 & 4.91 & -0.50 & -0.93 \\
\hline \multicolumn{17}{|l|}{ Comp. } \\
\hline Dist. 1 & 7.45 & 2.24 & $-1.19 *$ & 1.00 & 7.65 & 2.46 & -0.99 & 0.94 & 6.15 & 2.71 & -0.16 & -0.21 & 6.70 & 2.40 & -0.61 & 0.62 \\
\hline Dist. 3 & 8.15 & 2.21 & $-1.03 *$ & -0.58 & 7.85 & 2.46 & -0.88 & -0.29 & 6.55 & 2.96 & -0.98 & -1.62 & 6.55 & 2.98 & -0.47 & 1.07 \\
\hline
\end{tabular}

$* p<.05$ 


\section{Performance at post-test}

In the symbolic group, for verbal counting at post-test, four children counted to 100 , skewing the distribution. Accordingly, the post-test data were analyzed with the non-parametric Quade Test ${ }^{15}$ which revealed no differences at post-test between the two training conditions, $F(1,38)=0.19, M S E=46.86, p=.66, \eta^{2}{ }_{p}=.01$.

For the KeyMath numeration subtest, the ANCOVA assumption of no relation between the covariate and the independent variable was violated (i.e., there was a significant difference in performance between the training groups at pre-test), thus, a Quade Test was used to examine whether there were differences at post-test, the results showed no difference in performance between the two groups at post-test, $F(1,38)=$ $0.21, M S E=74.77, p=.65, \eta^{2}{ }_{p}=.01$.

Differences between the two groups at post-test for the six mapping tasks, and Give N, were analyzed with ANCOVAs controlling for the pre-test scores. For the verbal comparison task a MANOVA revealed no effect of training condition on distance 1 or distance 3 post-test items, $F(2,37)=1.14, p=.33, \eta^{2}{ }^{2}=.06$, therefore, an ANCOVA on the 20 items was performed to assess difference in performance at post-test. Results are summarized in Table 5.2. There were no differences in performance at post-test on any of the measures.

\footnotetext{
${ }^{15}$ The Quade Test involves ranking the pre- and post-test scores using SPSS Rank, regressing the ranked post-test scores on the ranked pre-test scores and then performing a 2(training group: symbolic, cardinality) ANOVA on the unstandardized residuals from the regression.
} 
Table 5.2. Statistics for Analyses of Covariance of numeracy performance controlling for pre-test scores.

\begin{tabular}{lccccccc}
\hline \multicolumn{1}{c}{ Measures } & \multicolumn{3}{c}{ Covariate } & \multicolumn{5}{c}{ Post-test } \\
\cline { 2 - 8 } & $F(1,37)$ & $p$ & $\eta^{2}{ }^{2}$ & $F(1,37)$ & $M S E$ & $p$ & $\eta^{2}{ }^{2}$ \\
\hline Mapping & & & & & & & \\
Digit-Quantity & 48.91 & .00 & .57 & 0.05 & 3.75 & .82 & .00 \\
Quantity-Digit & 22.29 & .00 & .38 & 0.00 & 7.41 & .97 & .00 \\
Number word-Quantity & 23.31 & .00 & .39 & 0.36 & 3.92 & .55 & .01 \\
Quantity-Number word & 21.28 & .00 & .37 & 0.11 & 6.22 & .75 & .00 \\
Number word-Digit & 39.70 & .00 & .52 & 0.07 & 2.17 & .80 & .00 \\
Digit-Number word & 11.06 & .00 & .23 & 1.22 & 4.46 & .27 & .03 \\
Other measures & & & & & & & \\
Cardinality & 49.83 & .00 & .57 & 0.45 & 0.37 & .51 & .01 \\
Verbal comparison & 49.86 & .00 & .57 & 0.01 & 9.69 & .92 & .00 \\
\hline
\end{tabular}

\section{Summary}

In summary, there were no differences at post-test between the two training conditions on any of the tasks. What could account for the lack of effects? First, an examination of the mean performance shows that the children in the present study already had good knowledge of the mapping between the number words and the digits in the range included in training, with an average score at pretest of around 16 out of 18 trials correct (almost 90\%). Thus, there was very little room for the children to improve on mapping number words to digits. Second, children in the study also showed good 
knowledge of cardinality at pre-test; on average, children in both conditions were able to accurately provide sets of five objects. Thus, many children had already mastered the cardinal principle of counting (Sarnecka \& Carey, 2008; Wynn, 1990; 1992).

Could training children in pairs have reduced the effect of the training? Children in the present study were observed to enjoy the games played with a partner. Although some researchers have pointed out that children may feel discouraged when the nature of the training procedure involves some form of competition (e.g., Ramani, Siegler, \& Hitti, 2012), this was not the case in the present study as the games did not encourage the children to be the "winners" and both children were awarded their stickers after each session. Furthermore, several intervention studies have successfully used small-group training procedures (i.e., groups of 2 to 4 children) to enhance young children's numeracy skills (e.g., Dyson, Jordan, \& Glutting, 2013; Krohn et al., 2012; Ramani et al., 2012; Toll \& VanLuit, 2012; Young-Loveridge, 2004). Thus, it is unlikely that the intervention carried out in pairs may have negatively affected the outcome of the training.

In summary, this study was planned based on the results of Study 1. However, the children recruited for this study were older, on average, and had good cardinality and digit naming knowledge. Thus, the training did not result in improvement in the trained skills and so there was no opportunity for it to transfer to the more complex mapping task (i.e., digit-quantity mapping) (See Figures 5.4 and 5.5). 
Figure 5.4. Results for pre- and post-test performance on digit-word mapping by training condition.

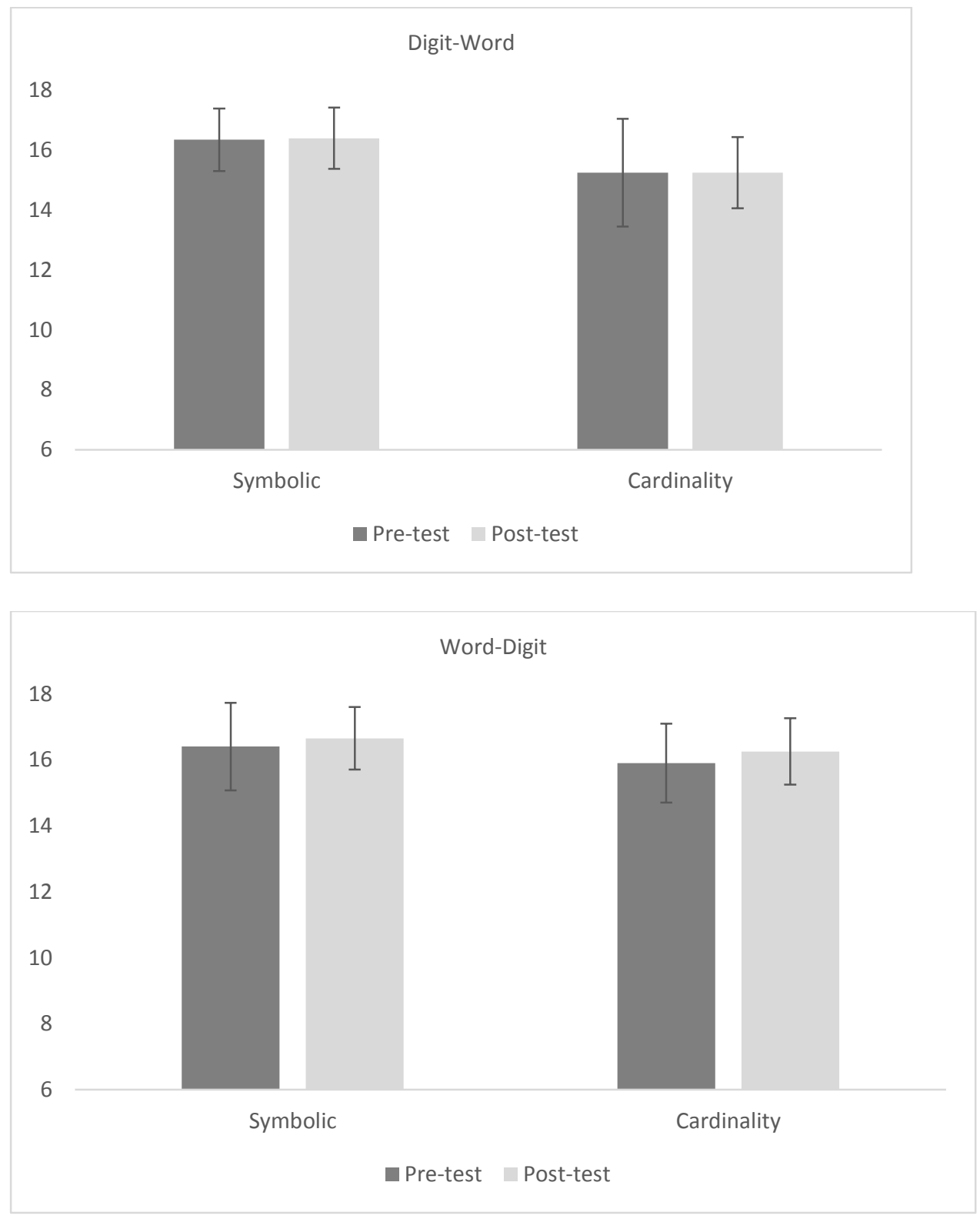


Figure 5.5. Results for pre- and post-test performance on digit-quantity mapping by training condition.
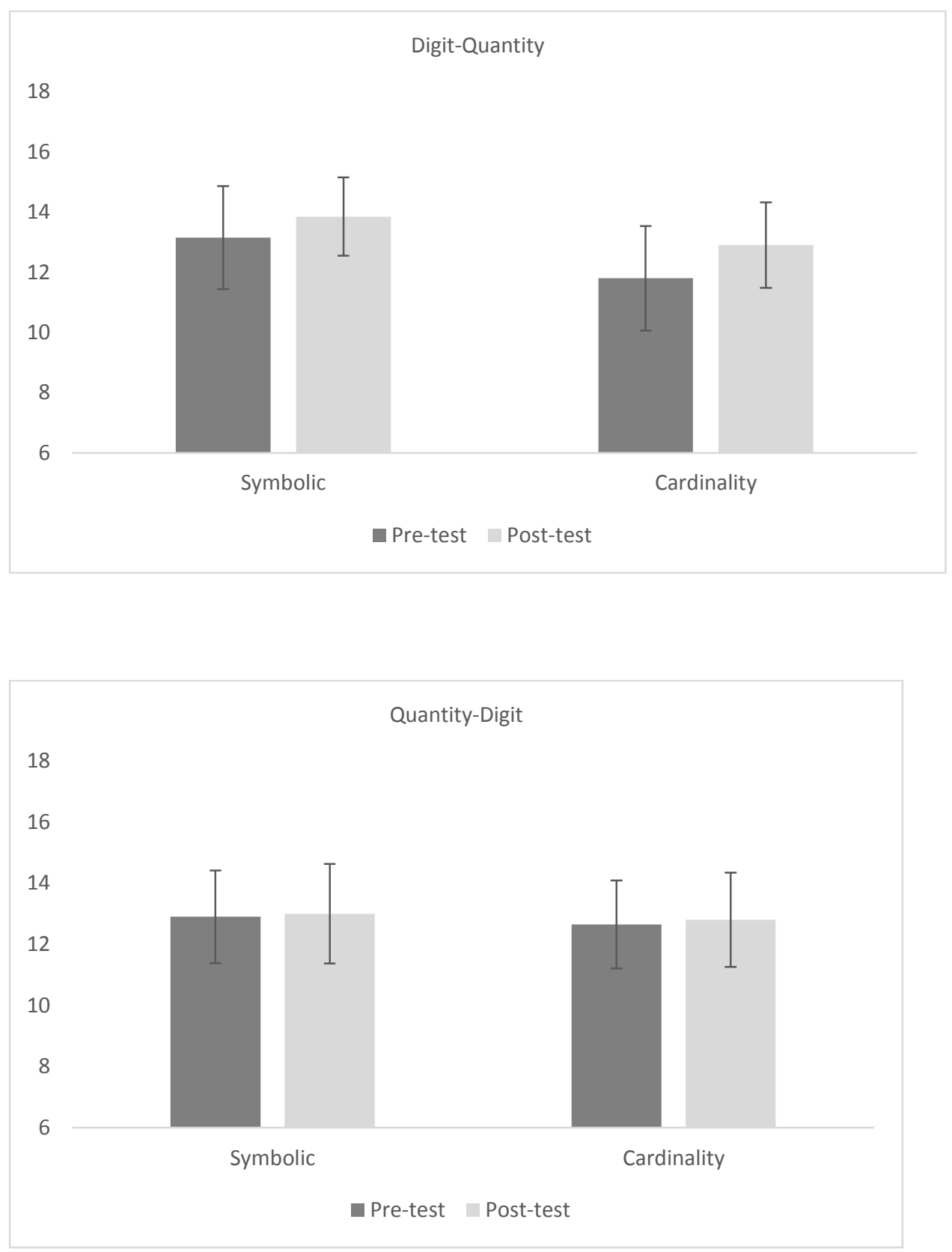


\section{CHAPTER 6: STUDY 2, HOME AND SCHOOL FACTORS \\ Introduction}

Results from Chapter 4 showed that Mexican children performed significantly worse than their Canadian counterparts on all the numeracy measures. Previous research conducted in Canada, the USA, Asia, and Europe has found that children's home learning environment (i.e., parental expectations, parent-reported frequency of engaging children in home numeracy and literacy activities) is related to children's developing numeracy skills (e.g., LeFevre et al., 2002; LeFevre et al., 2009; LeFevre, Polyzoi, et al., 2010; Kleemans et al., 2012; Manolitsis et al., 2013; Pan et al., 2006; Skwarchuk, 2009; Skwarchuk et al., 2014). Parents with higher academic expectations tend to report higher frequencies of engaging in home numeracy activities with their children (Kleemans et al., 2012; Segers et al., 2015); higher frequencies of home numeracy and literacy practices may benefit children's developing numeracy skills (e.g., LeFevre, et al., 2002; Manolitsis et al., 2013). The relationship between parental academic expectations, the frequency of engaging in home numeracy practices, and children's early numeracy skills has been assessed in cross-cultural research that included Canadian and Greek families (LeFevre, Polyzoi et al., 2010) and American and Asian families (Pan et al., 2006; Starkey \& Klein, 2008); however, to the best of my knowledge there is no published research conducted in Mexico on children's home learning environment and its relation to children's early numeracy skills.

Some research comparing numeracy performance and home numeracy practices between Mexican and North American children and parents has been conducted within the USA with samples of Mexican immigrants. These studies have found that Mexican 
children perform more poorly than North American and Asian children (e.g., Fei-Yin Ng, Tamis-LeMonda, Yoshikawa, \& Sze, 2014; Manfra, Dineheart, \& Sembiante, 2014). The lower performance displayed by Mexican children has been found to be related to Mexican parents' attitudes and beliefs about young children, their parenting practices, the frequency of home learning opportunities, and the type of childcare provided (Koury \& Votruba-Drzal, 2014).

Reese and Gallimore (2000) for example, found that immigrant Mexican parents believed that prior to the age in which children begin formal schooling, they are not capable of understanding early literacy concepts, and thus, reading to them or teaching them to print letters and words is not a useful practice. Reese and Gallimore reported that these parents began teaching their children literacy concepts only once they began school. Jung, Fuller, and Galindo (2012) found that Mexican immigrant mothers reported reading less to their children compared to White and Asian mothers. Tamis-LaMonda et al. (2013) found that Mexican mothers provided less feedback and taught numeracy concepts less often than Chinese and African American mothers during a block play session with their children.

The differences in early home practices that have been found between Mexican and North American and Asian parents could be related to differences in the underlying concept of education that Latino parents have (Reese, Balzano, Gallimore, \& Goldenberg, 1995). Latino parents believed that well educated, respectful, obedient children are more likely to be successful when they attend school, thus the emphasis of the home education is not on academic skills, but rather on morality and teaching what is right and wrong; learning academic skills is thus considered to be a responsibility of the 
school. Thus, one goal of the present research was to examine and compare Mexican and Canadian parents' academic expectations, the reported frequency of engaging in home numeracy and literacy practices, the relation between expectations and home practices, and how the home learning experiences are related to children's early numeracy skills.

Results from Chapter 4 also showed significant differences between Mexican and Canadian parents with regards to their highest level of education. More highly educated mothers have been found to provide their children with more enriching and cognitively stimulating activities; to communicate more frequently with their children's teachers, to have a better understanding of the development of their children's skills and abilities, and to be more aware of the school's goals and activities (Harding et al., 2015). Accordingly, there are significant correlations between maternal education and children's math performance (Gonzalez Estudillo, 2011; Purpura \& Reid, 2016). Furthermore, research has shown a significant correlation between parental education and income and the amount of math talk they use with their children (Gunderson et al., 2011; Levine et al., 2010). Thus, presumably, more highly educated mothers are able to provide a more enriched home learning environment which may benefit children's early numeracy skills. In the present study, maternal education was assessed as a predictor of the reported frequency of numeracy and literacy activities and of children's performance on the numeracy outcomes.

Children's math skills have been found to benefit from the amount of math input provided by preschool and daycare teachers (e.g., Boonen et al., 2011; Klibanoff et al., 2006). Although the guidelines for the numeracy skills that children are expected to acquire by age six are similar in both Canada and Mexico, the approach taken by 
daycares in Canada and preschools in Mexico is different. Canadian daycare teachers are not expected to directly teach children numeracy concepts, but instead they use a playbased approach to learning. Daycare teachers are encouraged to embed numeracy concepts within the context of children's daily routines (e.g., counting the cups during lunch time) and play activities (Ontario Ministry of Education, 2014). Mexican preschool teachers on the other hand, are expected to formally teach children specific math skills or concepts (SEP, 2011). Thus, a second goal of the present study was to describe children's math learning environment within their preschool or daycare centers in both countries.

In summary, the present study addressed the following research questions: a) do parental academic expectations and the reported frequency of engaging in home numeracy and literacy practices differ between Mexican and Canadian parents? b) Are parental academic expectations and maternal education predictive of the reported frequency of engaging in home numeracy and literacy activities in both countries? c) Are maternal education, academic expectations, and home numeracy and literacy practices related children's math outcomes in both countries? d) How do the daycare and preschool learning environments differ?

\section{Method}

\section{Participants}

A total of 54 Mexican and 40 Canadian parents returned the parent questionnaire. Thus, the response rate was $95 \%$ and $89 \%$ for the Mexican and Canadian parents, respectively. Children whose parents returned the questionnaire had a mean age of 47.5 and 47.6 months for the Mexican (31 girls) and Canadian (17 girls) children respectively. Mexican children were all monolingual Spanish speakers. A total of 20 Canadian 
children spoke another language in addition to English at home: seven children spoke French, 11 children spoke Chinese, and two children spoke Spanish.

Of the 54 Mexican parents who responded to the questionnaire, three completed the numeracy and literacy sections but did not complete the academic expectation section; and three completed the expectation section but were missing data on the numeracy and literacy sections of the questionnaire, finally two parents had missing data on the literacy practices section of the questionnaire only.

For the Canadian parents, three parents had missing data on both the literacy and numeracy sections, one parent had incomplete data on both the expectations and home numeracy and literacy practices sections of the questionnaire, one parent had missing data on the numeracy section, one had missing data on the literacy section.

\section{Results}

The results from parental reports of academic expectations and home numeracy and literacy activities in each country are first presented. I used multivariate and univariate analysis of variance to compare parental expectations between the two countries. Mixed ANOVA was used to assess the difference between countries in parentreported frequencies of numeracy and literacy practices. I used regression analyses to examine a) whether parental expectations are predictive of the reported frequency of home numeracy practices and b) the relationship between home numeracy and literacy practices and math outcomes. Maternal education was included as a predictor in all analyses. Finally, the data from the teacher questionnaire and the observed classroom visual stimuli are presented. 


\section{Do academic expectations differ between Mexican and Canadian parents?}

Parents' academic expectations were assessed with a questionnaire in which they had to respond on a five-point scale how important they considered it for their children to achieve six numeracy and seven literacy benchmarks before starting Grade 1 (see Table 5.1). Cronbach's Alpha for the thirteen items was .94 in the Mexican sample $(n=51)$ and .91 in the Canadian sample $(n=39)$. The differences between Mexican and Canadian parents on each item were analyzed with multivariate ANOVA and significant effects were followed up with univariate comparisons (see Table 6.1 for detailed comparisons). 
Table 6.1. Academic expectations by country.

\begin{tabular}{|c|c|c|c|c|c|c|}
\hline \multirow[t]{2}{*}{ Item } & \multicolumn{2}{|c|}{ Mexico } & \multicolumn{2}{|c|}{ Canada } & \multicolumn{2}{|c|}{ Difference } \\
\hline & $M$ & $S D$ & $M$ & $S D$ & $F(1,88)$ & $\eta_{p}^{2}$ \\
\hline \multicolumn{7}{|l|}{ Numeracy } \\
\hline Count to 10 & 3.22 & 0.76 & 3.51 & 0.76 & 3.41 & .04 \\
\hline Count to 100 & 2.57 & 1.17 & 2.03 & 1.09 & $5.05^{*}$ & .05 \\
\hline Read printed numbers up to 100 & 2.61 & 1.17 & 2.00 & 0.92 & $7.17 * *$ & .08 \\
\hline Know simple sums (e.g., 2+2) & 2.82 & 0.93 & 2.36 & 1.11 & $4.64 *$ & .05 \\
\hline Count to 1000 & 2.14 & 1.27 & 0.95 & 0.86 & $25.45^{* * *}$ & .22 \\
\hline Know multiplying (e.g., 2x6) & 2.23 & 1.27 & 0.85 & 0.93 & $32.85 * * *$ & .27 \\
\hline Literacy & $M$ & $S D$ & $M$ & $S D$ & $F(1,90)$ & $\eta_{p}^{2}$ \\
\hline Print his/her name & 3.45 & 0.78 & 3.23 & 0.90 & 1.61 & .02 \\
\hline Read a few words & 2.98 & 0.95 & 2.67 & 1.24 & 1.69 & .02 \\
\hline Print all the $26 / 27$ letters & 2.84 & 1.13 & 2.74 & 1.33 & 0.07 & .00 \\
\hline Know some alphabet letters & 3.31 & 0.76 & 3.64 & 0.71 & $4.85^{*}$ & .05 \\
\hline Know all 26/27 letters & 2.92 & 0.96 & 3.36 & 0.87 & $5.50 *$ & .06 \\
\hline Read simple picture books & 2.88 & 0.95 & 2.33 & 1.38 & $4.50^{*}$ & .05 \\
\hline Read chapter books & 2.51 & 1.15 & 0.90 & 1.10 & $45.48 * * *$ & .34 \\
\hline
\end{tabular}

$* p<.05, * * p<.01, * * * p<.001$.

${ }^{\text {a }}$ Spanish has the additional letter ñ.

Note. Parents responded on a five-point scale (0 to 4$)$ to the question: In your opinion, how important is it for your child to achieve the following benchmarks before starting Grade 1 ? Response options were 0 - unimportant, 1 - neither important nor unimportant, 2 - important, 3 - very important, and 4 - extremely important.

As shown in Table 6.1, Mexican parents had higher expectations than Canadian parents for all numeracy benchmarks, $F(6,83)=6.42, p<.001, \eta^{2}{ }_{p}=.32$ with one exception: both groups considered it very important that their children be able to count to 
10 before the start of Grade 1. In terms of literacy, Canadian parents reported higher expectations with regards to children being able to know the letters of the alphabet whereas Mexican parents reported higher expectations with regards to reading picture and even chapter books, $F(7,84)=14.04, p<.001, \eta^{2} p=.54$. However, parents in both countries had similar expectations regarding their children being able to print their names, print all the letters of the alphabet and read a few words.

These differences between the two groups of parents may indicate that Mexican parents were not very knowledgeable of the development of early literacy and numeracy skills in children. They may also reflect a social-desirability bias on the part of the Mexican parents. For further analyses a composite score of academic expectations was created by summing the 13 item scores.

\section{Are there differences between countries in the frequency with which parents report engaging in numeracy and literacy practices?}

To examine the home numeracy and literacy practices, the items were grouped into formal advanced, and formal basic numeracy practices following Skwarchuk et al. (2014; See Table 6.2). In their study, Skwarchuk and colleagues measured frequency of formal numeracy practices with a questionnaire that included 13 questions about how frequently parents engaged their children in the activities. In Skwarchuk et al., eight of the formal home numeracy questionnaire items loaded onto two factors (i.e., 4 items loaded onto each factor which were termed advanced and basic formal practices) the remaining five items that loaded on both factors in that study and therefore were grouped into a mixed category in the present research. Skwarchuk et al. assessed informal practices with a number game checklist that was not included in the present study. Thus, 
although the three factors include activities in which the activities are formal teaching experiences, the advanced formal factor contains items that tap into more complex tasks such as performing mental calculations or arithmetic operations. The basic items are less complex activities such as singing counting songs, playing board games, which do not require advanced number skills such as arithmetic. Finally, the mixed activities include both basic and advanced components such as playing number board games that include adding and subtracting.

Similarly, the questions on the literacy section of the parent questionnaire were grouped according to Skwarchuk et al. into formal advanced, formal basic, and mixed literacy practices. Skwarchuk et al assessed informal literacy with a storybook title checklist that was not included in the present study. Like the numeracy practices, the formal advanced literacy activities included activities which tapped into more complex skills such as reading words and identifying letters and words; formal basic activities included less complex activities such as singing or reciting the alphabet, the mixed literacy activities loaded on both factors and as with the numeracy practices.

Means, standard deviations and reliabilities for the formal advanced, formal basic, and mixed numeracy and literacy factors are presented in Tables 6.2 and 6.3. The factors had acceptable reliabilities. 
Table 6.2. Home numeracy practices by country.

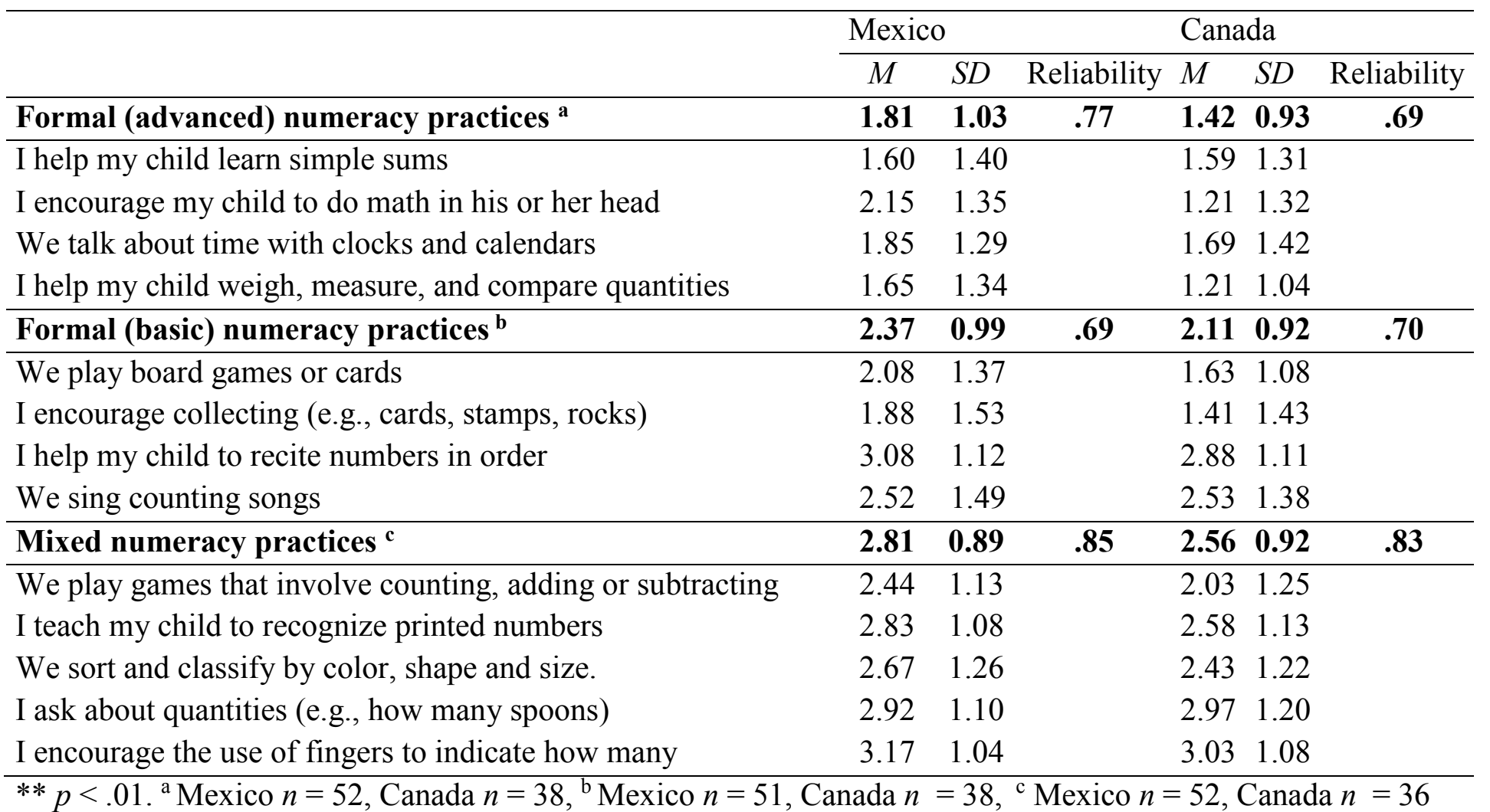

Note. Parents responded on a five-point scale to the question: How often do you do the following activities with your child? Response options were 0- rarely or never, 1- monthly, 2 - weekly, 3 - several days a week, and 4 - most days per week. In Skwarchuk et al. (2014), the mixed practices loaded on both the formal (advanced) and formal (basic) numeracy factors.

Reliability is Cronbach's Alpha based on the total number of items for the total number of parents with complete data. 
Table 6.3. Literacy home practice by country.

\begin{tabular}{|c|c|c|c|c|c|c|}
\hline & \multicolumn{3}{|c|}{ Mexico } & \multicolumn{3}{|c|}{ Canada } \\
\hline & $M$ & $S D$ & Reliability & $M$ & $S D$ & Reliability \\
\hline Formal Advanced a & $\mathbf{2 . 0 3}$ & 1.34 & .89 & 2.30 & 1.28 & .76 \\
\hline I help my child read words & 2.04 & 1.34 & & 2.33 & 1.42 & \\
\hline I ask my child to point to letters/words when we read & 2.06 & 1.48 & & 2.28 & 1.45 & \\
\hline Formal Basic ${ }^{b}$ & 2.18 & 1.11 & .72 & 2.47 & 1.11 & .79 \\
\hline I introduce new words and their definitions to my child & 2.58 & 1.35 & & 2.63 & 1.41 & \\
\hline I help my child sing/recite the alphabet & 1.73 & 1.36 & & 2.82 & 1.12 & \\
\hline We make up rhymes in songs (e.g., down by the bay) & 2.23 & 1.45 & & 2.05 & 1.41 & \\
\hline Mixed $^{\mathrm{c}}$ & 2.21 & $\mathbf{0 . 9 7}$ & .87 & 2.42 & 0.79 & .74 \\
\hline I teach my child to recognize printed letters & 3.02 & 1.02 & & 3.05 & 1.08 & \\
\hline I help my child print words & 2.19 & 1.39 & & 2.10 & 1.31 & \\
\hline We identify words on signs & 2.50 & 1.43 & & 2.23 & 1.42 & \\
\hline I teach my child the sounds of the letters & 2.67 & 1.20 & & 2.74 & 1.23 & \\
\hline I ask questions when we read together & 2.33 & 1.35 & & 2.88 & 1.14 & \\
\hline We visit the library for children's books & 0.58 & 1.11 & & 1.50 & 1.04 & \\
\hline
\end{tabular}

Note. Parents responded on a five-point scale to the question: How often do you do the following activities with your child? Response options were 0- rarely or never, 1- monthly, 2 - weekly, 3 - several days a week, and 4 - most days per week. Reliability is Cronbach's Alpha based on the items for the parents with complete data. 
To examine whether there were differences between countries in the reported frequency of literacy and numeracy activities, a 2(country) x 2 (domain: literacy, numeracy) x 3(type: formal advanced, formal basic, mixed) Mixed ANOVA was conducted on children's mean scores on each numeracy and literacy factor. Results are summarized in Table 6.4. There was a significant main effect of type of activity. Bonferroni corrected comparisons showed significant differences among the three types of activities (i.e., formal advanced, formal basic, and mixed). Across countries and domain (i.e., literacy and numeracy), parents report engaging most frequently on mixed $(M=2.48)$ activities than on formal basic $(M=2.27)$, and formal advanced $(M=1.89)$. The domain (i.e., literacy, numeracy) by type of activity (i.e., formal advanced, formal basic, and mixed) interaction provided a more complete picture: Across countries, there was a significant difference among the reported frequency of home numeracy practices, $F(2,164)=70.94, M S E=0.33, p<.001, \eta_{p}^{2}=.46$. Bonferroni corrected pairwise comparisons showed significant differences among formal advanced $(M=1.64)$, formal basic $(M=2.24)$, and mixed $(M=2.68)$ numeracy practices; in contrast, for the literacy activities, there was no difference in parent-reported frequency of engaging in formal advanced $(M=2.16)$, formal basic $(M=2.29)$, or mixed $(M=2.28)$ literacy activities, $F(2,164)=0.83, M S E=0.48, p=.44, \eta_{p}^{2}=.01$.

The significant domain (i.e., numeracy, literacy) by country interaction is shown in Figure 6.1. As can be seen in Figure 6.1, Mexican parents reported a slightly higher frequency of engaging in numeracy $(M=2.33)$ than in literacy $(M=2.11)$ home practices, $F(1,48)=5.99, M S E=0.20, p=.02, \eta^{2}=.11$. In contrast, Canadian parents 
reported engaging in literacy activities $(M=2.37)$ more frequently than in numeracy activities, $(M=2.02), F(1,33)=8.53, M S E=0.25, p=.01, \eta_{p}^{2}=.21$.

In summary there were significant differences in the frequency of home numeracy and literacy practices reported by parents in both countries. Replicating previous findings (e.g., Skwarchuk, 2009) Canadian parents reported engaging more frequently in literacy than in numeracy activities. Mexican parents on the other hand reported engaging their children more frequently in numeracy than in literacy activities. The finding that across countries parents reported that they engage less frequently in directly teaching their children more advanced numeracy skills (e.g., performing math in their heads, simple sums) and more often in formal basic and mixed practices is likely reflective of the children's young age.

Figure 6.1. Means for parent-reported frequencies of engaging in numeracy and literacy activities by country. Error bars are 95\% Confidence Intervals based on the MSE for the interaction.

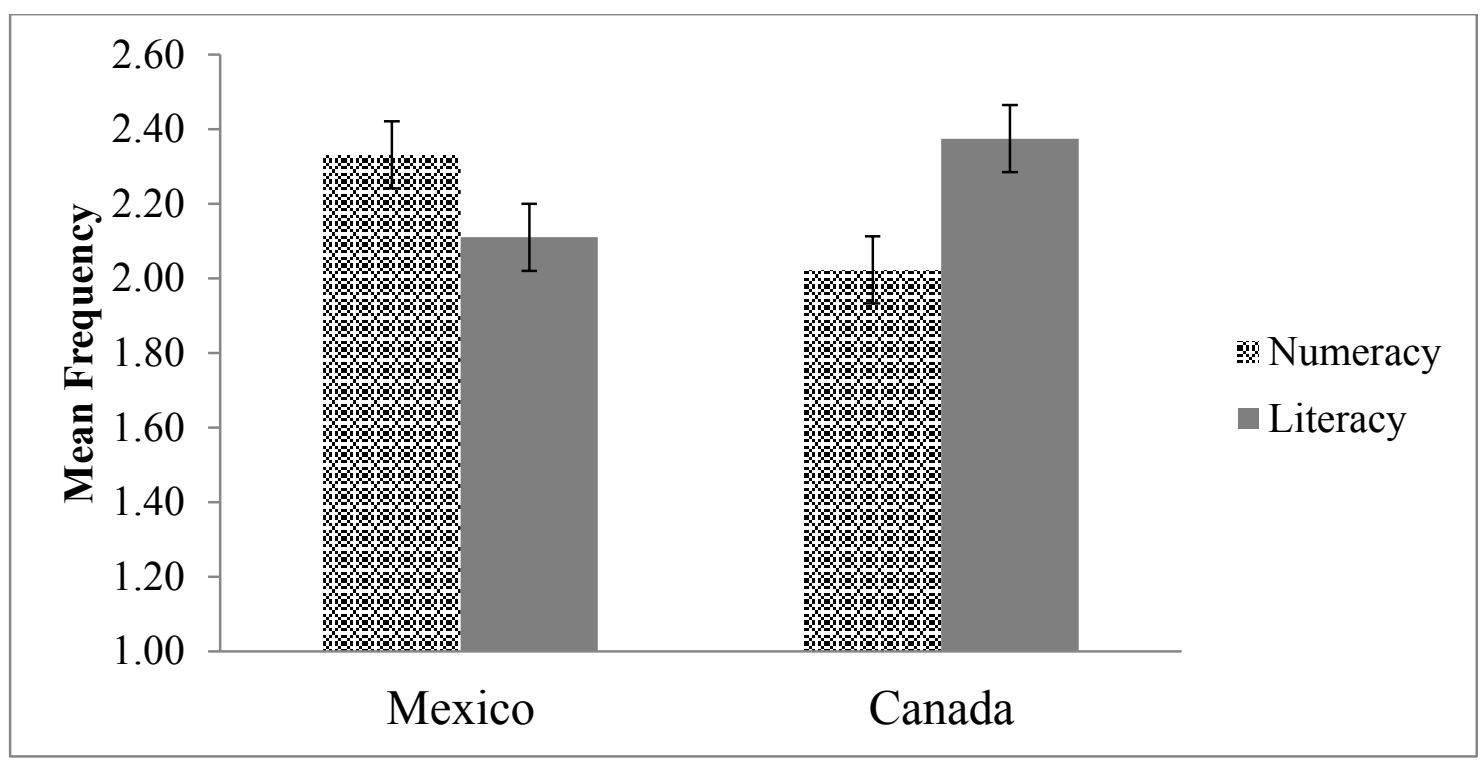


Table 6.4. Inferential statistics for analyses of home learning practices.

\begin{tabular}{lcccc}
\hline & $d f$ & $F$ & $M S E$ & $\eta^{2} p$ \\
\hline Country & 1,81 & 0.01 & 4.33 & .00 \\
Domain (numeracy, literacy) & 1,81 & 0.78 & 0.66 & .01 \\
Domain x country & 1,81 & $15.04^{* * *}$ & 0.66 & .16 \\
Type of activity (formal advanced, & 2,162 & $30.58^{* * *}$ & 0.47 & .27 \\
formal basic, mixed) & & & & \\
Type of activity x country & 2,162 & 0.05 & 0.47 & .00 \\
Domain x type of activity & 2,162 & $26.74^{* * *}$ & 0.34 & .25 \\
Domain x type of activity x country & 2,162 & 0.21 & 0.34 & .00 \\
\hline
\end{tabular}

Note: Mexican parents, $n=49$, Canadian parents, $n=34$

Are maternal education and parental academic expectations predictive of the reported frequency of engaging in home numeracy and literacy activities in both countries?

In prior research, higher parental education, especially the mother's education (Gonzales Estudillo, 2011; Harding et al., 2015), and parental expectations (Davis-Kean, 2005; LeFevre et al., 2009; Skwarchuk et al., 2014) were associated with higher math achievement and with the frequency with which parents report engaging in numeracy and literacy practices in preschool- and elementary-school children. Table 6.5 shows the correlations among maternal education, academic expectations, and numeracy and literacy practices in the Mexican and Canadian samples. Parents in both countries showed a similar pattern of inter-correlations among formal advanced, formal basic and mixed numeracy practices, and parental expectations. In both countries, the frequency of formal 
advanced numeracy practices was correlated with formal basic and mixed numeracy practices and with parental expectations; formal basic and mixed numeracy practices were highly intercorrelated but were not significantly correlated with parental expectations. Maternal education was not correlated with frequency of home numeracy activities or with parental expectations in either group of parents. Children's age was correlated with parents' report of formal advanced numeracy practices in the Canadian sample; in the Mexican sample, children's age did not correlate with any of the home numeracy practices.

The intercorrelations among parents' reported frequency of formal advanced, formal basic, and mixed literacy activities were high in the Mexican sample; in contrast, in the Canadian sample, formal advanced literacy practices were only correlated with mixed literacy practices, although formal basic and mixed literacy practices were highly intercorrelated. In both samples, formal advanced, formal basic, and mixed literacy practices were uncorrelated with maternal education, or academic expectations.

The intercorrelations among formal advanced, formal basic, and mixed numeracy and literacy practices were moderate to high in the Mexican sample; in the Canadian sample, however, the reported frequency of advanced literacy practices was uncorrelated with reported frequency of home numeracy practices. Thus, formal advanced literacy practices may be seen by Canadian parents as distinct from numeracy-related activities; in contrast in the Mexican sample, parents may view both practices as similar in that they both promote their children's development of early academic skills. Furthermore, in both samples, advanced, basic, and mixed literacy practices were uncorrelated with maternal education, or academic expectations. 
Table 6.5. Zero order correlations among education, academic expectations, and home practices. Canadian sample below the diagonal; Mexican sample above the diagonal.

\begin{tabular}{|c|c|c|c|c|c|c|c|c|c|}
\hline & \multicolumn{6}{|c|}{ Numeracy } & \multicolumn{3}{|c|}{ Literacy } \\
\hline & Age & Edu. & Exp. & $\begin{array}{c}\text { Formal } \\
\text { advanced }\end{array}$ & $\begin{array}{c}\text { Formal } \\
\text { basic }\end{array}$ & Mixed & $\begin{array}{c}\text { Formal } \\
\text { Advanced }\end{array}$ & $\begin{array}{c}\text { Formal } \\
\text { Basic }\end{array}$ & Mixed \\
\hline Age & - & -.07 & .10 & -.15 & -.04 & -.18 & -.17 & .01 & .02 \\
\hline Education & .25 & - & -.08 & -.01 & .05 & .14 & -.03 & .09 & -.04 \\
\hline Exp. & .25 & .11 & - & $.41 * *$ & .24 & .24 & .17 & .18 & $.27 \dagger$ \\
\hline \multicolumn{10}{|l|}{ Numeracy } \\
\hline Formal adv. & $.42 * *$ & .30 & $.43 * *$ & - & $.57 * *$ & $.65 * *$ & $.58 * *$ & $.66^{* *}$ & $.71 * *$ \\
\hline Formal basic & .12 & -.14 & .13 & $.44 * *$ & - & $.81^{* *}$ & $.60 * *$ & $.72 * *$ & $.70 * *$ \\
\hline Mixed & .01 & -.29 & .30 & $.55^{* *}$ & $.74 * *$ & - & $.51 * *$ & $.64 * *$ & $.71 * *$ \\
\hline \multicolumn{10}{|l|}{ Literacy } \\
\hline Formal adv. & .16 & .03 & .22 & .27 & .25 & $.32^{\dagger}$ & - & $.70 * *$ & $.75 * *$ \\
\hline Formal basic & -.12 & -.05 & .14 & $.34 *$ & $.70 * *$ & $.58 * *$ & .19 & - & $.83 * *$ \\
\hline Mixed & .18 & .07 & .17 & $.43 * *$ & $.70 * *$ & $.58 * *$ & $.73 * *$ & $.65 * *$ & - \\
\hline
\end{tabular}


To assess whether maternal education and parental expectations were predictive of the parent-reported frequency of home numeracy and literacy activities in both countries and to explore whether they would predict frequency of the same type of home numeracy and literacy activities in the Canadian and Mexican samples, regression analyses were performed. Results are presented in Tables 6.6 and 6.7.

Consistent with prior research (LeFevre et al., 2009; Skwarchuk et al., 2014) parents in both countries with higher expectations also reported engaging in formal advanced home numeracy practices more frequently (see Table 6.6). Frequency of home practices was not predicted by mothers' education or children's age. In contrast, parental academic expectations did not predict frequency of engaging in home literacy practices in either country (see Table 6.7).

Table 6.6. Results from regression analyses predicting home numeracy practices in both countries (unstandardized coefficients).

\begin{tabular}{lcccccc}
\hline \multicolumn{7}{c}{ Dependent Measures (Frequency of Home Numeracy Practices) } \\
\hline Predictors & \multicolumn{5}{c}{ Mexico } & \multicolumn{3}{c}{ Canada } \\
\cline { 2 - 7 } & $\begin{array}{c}\text { Formal } \\
\text { advanced }\end{array}$ & $\begin{array}{c}\text { Formal } \\
\text { basic }\end{array}$ & Mixed & $\begin{array}{c}\text { Formal } \\
\text { advanced }\end{array}$ & $\begin{array}{c}\text { Formal } \\
\text { basic }\end{array}$ & Mixed \\
\hline Intercept & 1.40 & 5.84 & 9.77 & -1.74 & 10.53 & 16.14 \\
Age ${ }^{\text {a }}$ & -0.11 & 0.05 & -0.04 & 0.18 & 0.08 & -0.01 \\
Education & 0.06 & 0.29 & 0.57 & 0.94 & -1.02 & -2.27 \\
Expectations & $0.16^{* *}$ & 0.09 & $0.10^{\dagger}$ & $0.14^{*}$ & 0.05 & $0.15^{\dagger}$ \\
\hline$R^{2}$ & $.18^{*}$ & .07 & .10 & $.33^{* *}$ & .06 & .18
\end{tabular}

${ }^{\mathrm{a}}$ Grand mean (i.e., 47.68 months) centered. ${ }^{\dagger} p<.063,{ }^{*} p<.05,{ }^{* *} p<.01$ 
Table 6.7. Results from regression analyses predicting home literacy practices in both countries (unstandardized coefficients).

Dependent Measures (Frequency of Home Literacy Practices)

\begin{tabular}{lcccccc}
\hline & \multicolumn{3}{c}{ Mexico } & & \multicolumn{3}{c}{ Canada } \\
\cline { 2 - 7 } Predictors & $\begin{array}{c}\text { Formal } \\
\text { advanced }\end{array}$ & $\begin{array}{c}\text { Formal } \\
\text { basic }\end{array}$ & Mixed & $\begin{array}{c}\text { Formal } \\
\text { advanced }\end{array}$ & $\begin{array}{c}\text { Formal } \\
\text { basic }\end{array}$ & Mixed \\
\hline Intercept & 2.65 & 4.52 & 8.82 & 2.77 & 6.06 & 11.29 \\
Age $^{\text {a }}$ & -0.07 & 0.06 & 0.13 & 0.08 & -0.09 & 0.12 \\
Education & -0.07 & 0.14 & -0.19 & 0.17 & -0.18 & 0.32 \\
Expectations & 0.05 & 0.05 & 0.14 & 0.05 & 0.06 & 0.07 \\
\hline$R^{2}$ & .04 & .04 & .09 & .09 & .05 & .05 \\
\hline
\end{tabular}

Are maternal education, expectations, and home numeracy, literacy practices related to children's performance on numeracy assessments?

The correlations between child performance on the numeracy measures and maternal education, parental expectations and home numeracy and literacy practices are presented in Table 6.8 for the Mexican sample and in Table 6.9 for the Canadian sample. In Mexico, maternal education was correlated with measures that involved knowledge of the number words and the quantities that they represent (i.e., Give N, word-quantity mapping, and verbal comparison). In Canada, maternal education was correlated with all math measures except word-digit mapping. Researchers have proposed that maternal education affects children's academic outcomes through mechanisms such as maternal cognitive skills and vocabulary which are more advanced in more highly educated mothers; thus more highly educated mothers are able to engage their children in 
cognitively stimulating conversations (and activities) which in turn benefit their academic skills. In addition, more highly educated mothers have been found to interact more with the teachers and are more likely to request support from the teachers when they feel their child requires additional support (Harding et al., 2015; Lareau, 2011). Thus, even though there was no relation between maternal education and frequency of numeracy or literacy activities (see Tables 6.6 and 6.7), maternal education may be related to performance through these other proposed mechanisms, the question is therefore open to future research.

As shown in Table 6.8, in Mexico, children's performance on the early numeracy assessments were uncorrelated with parental reports of frequency of formal advanced, formal basic, or mixed numeracy practices. Similarly, Mexican children's performance on the numeracy measures was uncorrelated with parental expectations. For literacy practices, however, there was a significant negative correlation between parents' reports of formal advanced literacy practices and children's performance on the Word-Quantity and Give-N Tasks. Overall, therefore, these results are not similar to those in previous research with children from other countries.

As shown in Table 6.9, for the Canadian sample, parents' reported frequency of formal advanced numeracy practices was significantly correlated with all numeracy measures except Give N. Formal advanced literacy practices was significantly and positively correlated with children's performance on the KeyMath; mixed literacy practices was correlated with children's performance on the Give N task. Parents' reported academic expectations were significantly correlated with children's performance on the digit-quantity mapping measure. 
Table 6.8. Mexican sample zero order correlations among tasks and home numeracy practices (mapping combined orders).

\begin{tabular}{|c|c|c|c|c|c|c|c|c|}
\hline & & & \multicolumn{3}{|c|}{ Numeracy } & \multicolumn{3}{|c|}{ Literacy } \\
\hline & M. Edu & Expect. & $\begin{array}{c}\text { Formal } \\
\text { advanced }\end{array}$ & $\begin{array}{c}\text { Formal } \\
\text { basic }\end{array}$ & Mixed & $\begin{array}{c}\text { Formal } \\
\text { advanced }\end{array}$ & $\begin{array}{c}\text { Formal } \\
\text { basic }\end{array}$ & Mixed \\
\hline Age & -.07 & .10 & -.15 & -.04 & -.18 & -.17 & .01 & .02 \\
\hline High count & .15 & .12 & -.19 & -.15 & -.13 & -.12 & -.05 & -.01 \\
\hline Digit-quantity & .12 & -.00 & -.07 & -.09 & .08 & -.09 & .01 & .15 \\
\hline Word-quantity & $.34^{*}$ & -.07 & $-.27 \dagger$ & -.21 & -.07 & $-.30 *$ & -.13 & -.08 \\
\hline Word-digit & .24 & .06 & .08 & .10 & .15 & .12 & .20 & $.27 \dagger$ \\
\hline Give N & $.33^{*}$ & .03 & -.14 & -.11 & .02 & $-.29 *$ & -.08 & -.03 \\
\hline KeyMath & .25 & .15 & -.06 & -.10 & .05 & -.05 & -.02 & .03 \\
\hline Verbal Comparison & $.33^{*}$ & .09 & .04 & -.03 & .14 & .02 & .06 & .17 \\
\hline
\end{tabular}

$\dagger p<.054$ 
Table 6.9. Canadian sample zero correlations among tasks and home numeracy practices (mapping combined).

\begin{tabular}{|c|c|c|c|c|c|c|c|c|}
\hline & & & \multicolumn{3}{|c|}{ Numeracy } & \multicolumn{3}{|c|}{ Literacy } \\
\hline & M. Edu & Expect. & $\begin{array}{c}\text { Formal } \\
\text { advanced }\end{array}$ & $\begin{array}{c}\text { Formal } \\
\text { basic }\end{array}$ & Mixed & $\begin{array}{c}\text { Formal } \\
\text { advanced }\end{array}$ & $\begin{array}{c}\text { Formal } \\
\text { basic }\end{array}$ & Mixed \\
\hline Age & .25 & .25 & $.42 * *$ & .12 & .01 & .16 & -.12 & .18 \\
\hline High count & $.47 *$ & .22 & $.45^{*}$ & .03 & -.05 & $.31 \dagger$ & .02 & .22 \\
\hline Digit-quantity & $.30 *$ & $.32 *$ & $.44 * *$ & .27 & .27 & .28 & -.01 & .20 \\
\hline Word-quantity & $.35^{*}$ & .17 & $.36^{*}$ & .20 & .19 & .20 & -.13 & .10 \\
\hline Word-digit & .27 & .19 & $.48 * *$ & .09 & .23 & .22 & -.02 & .13 \\
\hline Give N & $.32 *$ & .24 & .28 & $.31 \dagger$ & .22 & .24 & .16 & $.42 *$ \\
\hline KeyMath & $.45 * *$ & .26 & $.42 * *$ & .20 & .14 & $.37 *$ & -.09 & $.31 \dagger$ \\
\hline Verb. comparison & $.41 * *$ & .29 & $.47 * *$ & .28 & .14 & .14 & -.06 & .15 \\
\hline
\end{tabular}


To determine whether parents' reports of numeracy and literacy practices were related to children's performance as in previous work, regression analyses predicting each numeracy outcome from age, maternal education, parental expectations, formal advanced, and formal basic home numeracy practices and formal advanced literacy practices were performed. Mixed numeracy practices and formal basic literacy practices were uncorrelated with the numeracy outcomes in both countries and were excluded from the analyses. In addition, mixed literacy practices showed high correlations with formal basic numeracy in both countries and was therefore also excluded from the subsequent analyses. Results are presented in Table 6.10, 6.11, and 6.12.

Table 6.10 shows the analyses for verbal counting and cardinality. For the Canadian children, verbal counting was only predicted by maternal education. Canadian children's cardinality skills were predicted by both formal basic numeracy practices and maternal education. In the Mexican sample, only age significantly predicted children's verbal counting and maternal education predicted children's cardinality skills. Parental reports of the frequency of engaging in home numeracy and literacy practices were unrelated to Mexican children's verbal counting and cardinality skills.

Table 6.11 summarizes the results of the regression analyses predicting performance on the three mapping tasks. For the Canadian children, formal advanced numeracy practices were marginally associated with children's word-digit mapping. The symbol-quantity mapping tasks were unrelated to parental reports of home numeracy and literacy practices. For the Mexican children, maternal education significantly predicted word-quantity mapping. 
Table 6.12 shows the analyses for children's more complex math skills, that is, the KeyMath numeration subtest and verbal comparison. Canadian children's performance on the KeyMath was significantly related to maternal education. Performance on the verbal number comparison task was unrelated to maternal education and parental reported frequency of engaging in home numeracy and literacy practices. Mexican children's performance on the KeyMath was only predicted by the children's age, performance on the verbal comparison task was significantly predicted by maternal education.

Maternal education was the most consistent predictor of performance, accounting for unique variance in both samples. For the Mexican children maternal education predicted performance on the verbal comparison, Give N, and word-quantity mapping; for the Canadian children maternal education predicted performance on the KeyMath, Give N, and verbal counting. Parents' reports of home practices did not account for variance in any outcomes for the Mexican group. In contrast, in the Canadian group parent reported frequency of engaging in numeracy practices were related to children's cardinality knowledge and marginally to their knowledge of the word-digit mapping. 
Table 6.10. Results from regression analyses predicting counting outcomes for the Canadian and Mexican sample.

\begin{tabular}{|c|c|c|c|c|c|c|c|c|}
\hline & \multicolumn{4}{|c|}{ Canada } & \multicolumn{4}{|c|}{ Mexico } \\
\hline & \multicolumn{2}{|c|}{ Count } & \multicolumn{2}{|c|}{ Give $\mathrm{N}$} & \multicolumn{2}{|c|}{ Count } & \multicolumn{2}{|c|}{ Give $\mathrm{N}$} \\
\hline & $\mathrm{B}$ & $\beta$ & $\mathrm{B}$ & $\beta$ & $\mathrm{B}$ & $\beta$ & $\mathrm{B}$ & $\beta$ \\
\hline Intercept & -12.79 & & -0.25 & & $6.85 * *$ & & $2.55^{*}$ & \\
\hline Age & 0.47 & 0.26 & $0.12^{* *}$ & $0.49 * *$ & $0.33 * *$ & $0.44 * *$ & $0.13^{\dagger}$ & $0.29^{\dagger}$ \\
\hline Education & $7.05 * *$ & $0.40 * *$ & $1.03 * *$ & $0.42 * *$ & 0.45 & 0.17 & $0.54 *$ & $0.34 *$ \\
\hline Expectation & -0.00 & -0.00 & 0.03 & 0.16 & 0.06 & 0.21 & 0.01 & 0.08 \\
\hline \multicolumn{9}{|c|}{ Home numeracy } \\
\hline Formal adv. & 0.76 & 0.27 & -0.13 & -0.31 & -0.15 & -0.18 & -0.03 & -0.06 \\
\hline Formal basic & -0.29 & -0.10 & $0.17^{*}$ & $0.43^{*}$ & -0.10 & -0.12 & 0.01 & 0.03 \\
\hline \multicolumn{9}{|c|}{ Home literacy } \\
\hline Formal adv. & 0.64 & 0.15 & 0.04 & 0.07 & 0.10 & 0.09 & -0.16 & -0.22 \\
\hline$R^{2}$ & $.49 * *$ & & $.52 * *$ & & $.27^{\dagger}$ & & $.26^{\dagger}$ & \\
\hline
\end{tabular}


Table 6.11 Results from regression analyses predicting mapping outcomes.

\begin{tabular}{|c|c|c|c|c|c|c|c|c|c|c|c|c|}
\hline & \multicolumn{6}{|c|}{ Canada } & \multicolumn{6}{|c|}{ Mexico } \\
\hline & \multicolumn{2}{|c|}{ Digit-qty } & \multicolumn{2}{|c|}{ Word-qty } & \multicolumn{2}{|c|}{ Word-digit } & \multicolumn{2}{|c|}{ Digit-qty } & \multicolumn{2}{|c|}{ Word-qty } & \multicolumn{2}{|c|}{ Word-digit } \\
\hline & $\mathrm{B}$ & $\beta$ & B & $\beta$ & $\mathrm{B}$ & $\beta$ & $\mathrm{B}$ & $\beta$ & $\mathrm{B}$ & $\beta$ & $\mathrm{B}$ & $\beta$ \\
\hline Intercept & $7.61^{*}$ & & $10.08 * *$ & & $13.89 * * *$ & & $11.20 * * *$ & & $12.68 * * *$ & & $11.24 * * *$ & \\
\hline Age & $.16^{\dagger}$ & $33^{\dagger}$ & $15^{*}$ & $.38^{*}$ & .11 & .23 & $.28 * *$ & $.43 * *$ & $.29 * *$ & $.39 * *$ & $.29^{*}$ & $.34 *$ \\
\hline Edu. & .56 & .12 & .63 & .17 & .31 & .07 & .34 & .15 & $.87 *$ & $.35^{*}$ & .47 & .16 \\
\hline Exp. & .03 & .10 & .00 & .00 & -.02 & -.07 & -.02 & -.07 & -.01 & -.03 & -.03 & -.10 \\
\hline \multicolumn{13}{|c|}{ Home Numeracy } \\
\hline $\begin{array}{l}\text { Formal } \\
\text { adv. }\end{array}$ & .10 & .13 & .02 & .03 & $.31^{\dagger}$ & $.41^{\dagger}$ & -.02 & -.03 & -.17 & -.21 & .07 & .08 \\
\hline $\begin{array}{l}\text { Formal } \\
\text { basic }\end{array}$ & .08 & .11 & .10 & .16 & -.12 & -.16 & -.05 & -.07 & -.03 & -.04 & .03 & .03 \\
\hline \multicolumn{13}{|c|}{ Home Literacy } \\
\hline $\begin{array}{l}\text { Formal } \\
\text { adv. }\end{array}$ & .19 & .17 & .07 & .08 & .16 & .15 & -.01 & -.01 & -.13 & -.12 & .16 & .12 \\
\hline$R^{2}$ & $.34^{*}$ & & .27 & & .30 & & .20 & & $.37 * *$ & & .16 & \\
\hline
\end{tabular}


Table 6.12. Results from regression analyses predicting more complex math outcomes for the Canadian and Mexican sample.

\begin{tabular}{|c|c|c|c|c|c|c|c|c|}
\hline & \multicolumn{4}{|c|}{ Canada } & \multicolumn{4}{|c|}{ Mexico } \\
\hline & \multicolumn{2}{|c|}{ KeyMath } & \multicolumn{2}{|c|}{ Verbal Comp. } & \multicolumn{2}{|c|}{ KeyMath } & \multicolumn{2}{|c|}{ Verbal Comp. } \\
\hline & $\mathrm{B}$ & $\beta$ & $\mathrm{B}$ & $\beta$ & $\mathrm{B}$ & $\beta$ & $\mathrm{B}$ & $\beta$ \\
\hline Intercept & $-3.50 * *$ & & $9.06^{\dagger}$ & & -.33 & & $7.58 * *$ & \\
\hline Age & $.06^{*}$ & $.34^{*}$ & $.46^{* * *}$ & $.62 * * *$ & $.10^{*}$ & $.40^{*}$ & .26 & .25 \\
\hline Education & $.69 * *$ & $.41 * *$ & .73 & .10 & .22 & .25 & $1.44 * *$ & $.40 * *$ \\
\hline Expectation & .01 & .09 & .03 & .07 & .01 & .14 & .04 & .10 \\
\hline \multicolumn{9}{|c|}{ Home numeracy } \\
\hline Formal adv. & .00 & .01 & .09 & .07 & -.02 & -.06 & .04 & .04 \\
\hline Formal basic & .05 & .18 & .22 & .19 & -.06 & -.23 & -.30 & -.27 \\
\hline \multicolumn{9}{|c|}{ Home literacy } \\
\hline Formal adv. & .08 & .19 & -.14 & -.08 & .06 & .15 & .26 & .17 \\
\hline$R^{2}$ & $.49 * *$ & & $.52 * *$ & & .23 & & .22 & \\
\hline
\end{tabular}




\section{Summary}

The first goal of the present study was to examine parents' academic expectations and their reported frequency of engaging in numeracy and literacy practices with their children both in Canada and Mexico. Results showed significant differences between the two countries in parents' reported academic expectations. Mexican parents reported higher expectations than Canadian parents for all numeracy benchmarks (except count to 10). For literacy expectations, Mexican parents reported higher expectations on the more advanced items such as reading chapter books. The differences probably reflect Mexican parents' lack of knowledge of the development of children's early numeracy and literacy skills. This conclusion is supported by the finding that Mexican parents rated the most difficult numeracy and literacy skills as 'very important' (e.g., know multiplying, counting to 1000, and reading chapter books). Those are unrealistic expectations for children at the end of Kindergarten, especially given the Mexican children had less advanced numeracy skills than the Canadian children. In both countries, academic expectations were moderately predictive of the frequency with which parents reported engaging in formal advanced numeracy practices. Of interest was the finding that, contrary to previous research with parents and children in Canada (Skwarchuk et al., 2014), academic expectations were unrelated to parent-reported frequencies of formal advanced, basic, and mixed literacy practices.

With regards to parents' reports of numeracy and literacy practices, consistent with past research (e.g., Blevins Knabe \& Musun-Miller, 2000; LeFevre, et al., 2009; Skwarchuk, 2009) Canadian parents reported engaging more frequently in literacy than numeracy activities. Mexican parents on the other hand, reported engaging more 
frequently in numeracy than in literacy activities. The finding that Mexican parents report engaging more frequently in numeracy than literacy could reflect the effect of parents' interaction with their children's teachers. For example, in a study including low income Latino immigrants to the USA, Reese and Gallimore, (2000) reported that once children began school and parents came in contact with the teachers' goals and school practices, parents began engaging in home practices to comply with the teacher. This explanation would also be consistent with the finding that parents' reports of home practices are negatively correlated with children's performance: Perhaps parents of those children who were doing most poorly were more likely to be approached by teachers with suggestions for home activities.

Research has also found that parental involvement in school is related to parents' awareness of the importance of learning activities at home (Hill \& Taylor, 2004). In the present study, anecdotal evidence from the author shows that teachers were very concerned about their children's numeracy development. Furthermore, the teachers mentioned that they encouraged the parents to teach their children math at home. Of note with respect to literacy practices, prior research has found that parents whose language involves a more regular orthography report engaging less frequently in literacy activities (e.g., Manolitsis et al., 2013). In Spanish, there is a transparent correspondence between letters and their sounds. Research suggests that learning to read in Spanish may be easier than learning to read in English (Aro \& Wimmer, 2003). Thus, it is possible that because teachers are aware that learning to read is relatively easy they therefore emphasize and encourage parents to focus more on numeracy. Thus, Mexican parents' responses to the 
questionnaires could reflect that they are aware that they should be engaging their children in numeracy activities, rather than reflecting established attitudes and practices.

A second goal of the study was to assess the relationship between maternal education, parental academic expectations, parent reported frequency of engaging in home learning activities and children's performance on the early numeracy assessments. Results from the regression analyses show that the only demographic predictor of children's numeracy outcomes in the Mexican sample was maternal education. It significantly predicted children's performance on the Give N, word-quantity mapping, and verbal comparison tasks and was positively related to most of the other numeracy skills. In contrast, reports of home practices were not related to performance on any of the measures. In the Canadian sample, maternal education predicted performance on verbal counting, the Give N task, and on the KeyMath Numeration Subtest. Canadian children's performance on the Give $\mathrm{N}$ task was significantly predicted by parent-reported frequency of formal basic numeracy and performance on the word-digit mapping was marginally predicted by parental reports of frequency of engaging in formal advanced numeracy activities. Inclusion of the home literacy variables in the same analyses (along with small sample sizes) may have limited the power to detect small effects because the simple correlations between parents' reports of home numeracy practices and children's performance were similar or higher than those in other studies.

The finding that maternal education was related to children's knowledge of the meaning of the number words (and knowledge of the symbolic number system in general in the Canadian sample) is consistent with prior research by Gunderson et al. (2011) and Levine et al. (2010) who found that a factor created from parent education and income 
was positively correlated with the amount of math talk that parents engaged with their children. Both studies found a significant relationship between the amount of math talk provided by the caregivers and children's performance on a cardinality assessment. In addition, more complex math talk (e.g., counting larger sets of objects) is predictive of children's performance on more advanced math skills such as magnitude understanding (Ramani et al., 2015), rather than math talk about smaller quantities. Thus, more highly educated mothers may be more knowledgeable of the development of children's early numeracy skills and engage in more frequent and more complex math talk.

In the Canadian sample, parents' reports of the frequency of formal basic numeracy practices significantly predicted children's performance on the Give N cardinality assessment. In Skwarchuk et al., (2014) the reported frequency of engaging in formal basic activities were unrelated to both knowledge of the symbolic number system and non-symbolic math skills, however, the children in that study were almost a year older than the children in the present study at the time of testing. In the present study, however, these basic activities may have been related to cardinality performance because they are developmentally appropriate for cardinality learning.

Finally, the finding that parents' reported frequency of formal advanced numeracy practices was marginally related to children's ability to map number words to digits is consistent with prior research and shows that parents' reports of the frequency of directly teaching their children more advanced math skills is related to their knowledge of the symbolic number system (LeFevre et al., 2002; Skwarchuk et al., 2014). 


\section{Preschool and daycare learning environments}

A goal of the present study was to describe children's preschool or daycare learning environments. The classrooms in both countries were examined for the presence or absence of numeracy and literacy visual stimuli. Numeracy visual stimuli included a) presence of a clock with the numbers big enough for children to see, b) presence of a calendar, c) Arabic digits displayed on the wall d) whether the digits were arranged in sequential order, and e) whether the quantities corresponding to the digits were also displayed. Table 6.13 shows a summary of the presence of numeracy artifacts in the classrooms in both countries.

Table 6.13. Percentage of Daycares/Preschools with of Literacy and Numeracy Artifacts

\begin{tabular}{lll}
\hline Visual Artifacts & Mexico Canada
\end{tabular}

\section{Numeracy}

Clock on the wall

20

100

Calendar on the wall

40

67

Have the Arabic digits

60

83

Books that include numbers

60

100

\section{Literacy}

Letters on the wall

80

67

Words on the wall

80

67

As can be observed from Table 6.13, the Canadian daycares had more visual numerical stimuli and when the numbers were displayed in some cases they were larger. For example, five Canadian daycare classrooms displayed the digits on the wall; of the 
five classrooms that displayed the digits on the wall, three had all the numbers $1-10$, one had all the numbers 1-20, these numbers were arranged in sequential order; the fifth one only had the numbers 4 and 6 ; the corresponding quantities in the form of various pictures (e.g., fruits, bears, stick figures) were always displayed next to each digit. In Mexico, three of the classrooms displayed the numbers on the wall all of which included the whole range of 1-10 arranged in sequential order; only two of the classrooms displayed the corresponding quantities: one was a caterpillar which had the paper balls beneath each number (see Figure 6.2), the other had either one or two hands holding up the corresponding number of fingers.

The number of available books within the classrooms was also greater in the Canadian daycares. All the daycares had books that included number within the classroom; the number of books ranged from 2 to 200. In Mexico, of the five classrooms, three had number books: two classrooms had 10 books each and one had 5 . Figure 6.2. Caterpillar displaying the written Arabic digits and the corresponding quantities.

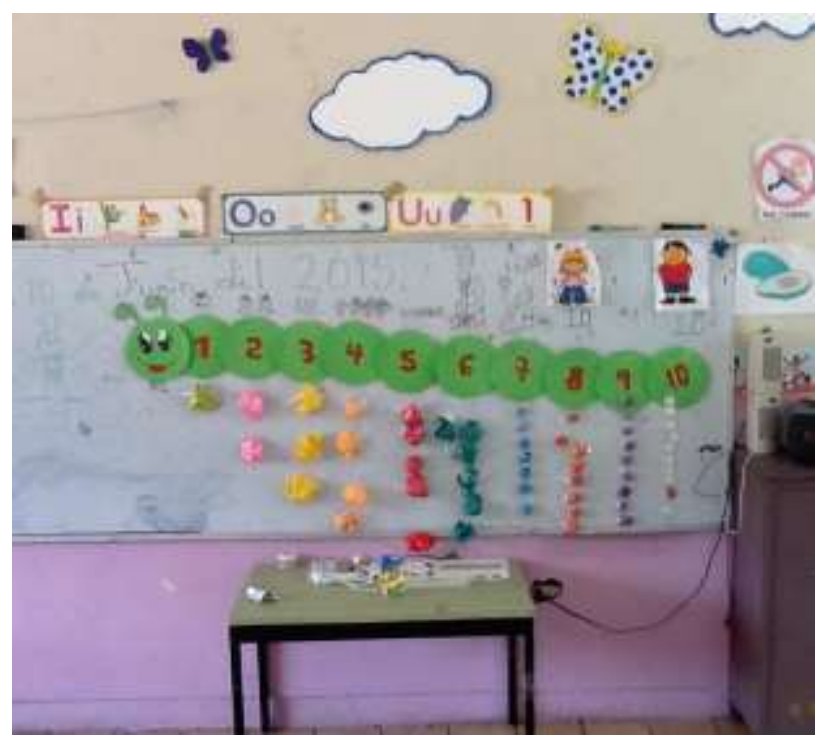


Table 6.13 also shows the percentage of classrooms that had letters and words posted on the walls. In Canada, the letters that were posted in the four classrooms were the 26 letters of the alphabet. In Mexico, one classroom had the entire alphabet, two classrooms had the vowels, and one classroom had the first letter of each of the children's names. The Mexican classrooms that had words posted on the walls had the children's names, the months of the year, the colors, the parts of the flowers, and signs such as "wash your hands". In Canada, the words that were posted were similar to those in the Mexican classrooms including days and months, names of flowers and animals, and messages such as "Be kind" "Be positive" and "Be fair."

In summary, Canadian daycares had more resources than Mexican preschools, including more books, clocks, calendars. The numerical visual stimuli displayed in Canadian daycares included larger numbers than in Mexican preschools (where one classroom even had bare walls). In addition, artifacts that display numbers such as clocks and calendars were present in more classrooms than in Mexican preschools. With regards to literacy stimuli, the percentage of classrooms that displayed words and letters on the wall was slightly higher in Mexico than in Canada.

\section{Numeracy and literacy activities reported by teachers}

Teachers in both countries were asked to respond to 19 questions about frequency of numeracy practices conducted within the classroom. The numeracy questionnaire included five sections in which teachers were asked to report the frequency of activities related to a) digit-word mapping, b) quantity-word mapping, c) quantity-digit mapping, d) general math knowledge, and e) number comparison. In addition, teachers completed 10 questions about classroom literacy practices. Teachers responded to each question 
using a scale from 0 to 4 . Table 6.14 and 6.15 presents the questionnaire items included in each section and the overall item means by country.

The distribution of responses for six of the numeracy items provide some insight into the difference in approach to teaching taken by Canadian daycares and Mexican preschools. For example, as shown in Figure 6.3, Canadian teachers reported a greater frequency of engaging the children in more basic numeracy activities such as a) singing number songs, b) using books to teach about number, and c) using objects to teach the children about more complex math concepts such as fractions. In contrast, Mexican teachers reported more frequently a) asking the children to complete worksheets that require them to verbally count objects, b) complete worksheets that depict the written Arabic digit and the corresponding quantity, and c) ask children to count and write the digit (See Figure 6.4) consistent with a more formal direct approach to teaching early math. 
Figure 6.3. Distribution of responses for items on which Canadian teachers reported greater frequencies than Mexican teachers.
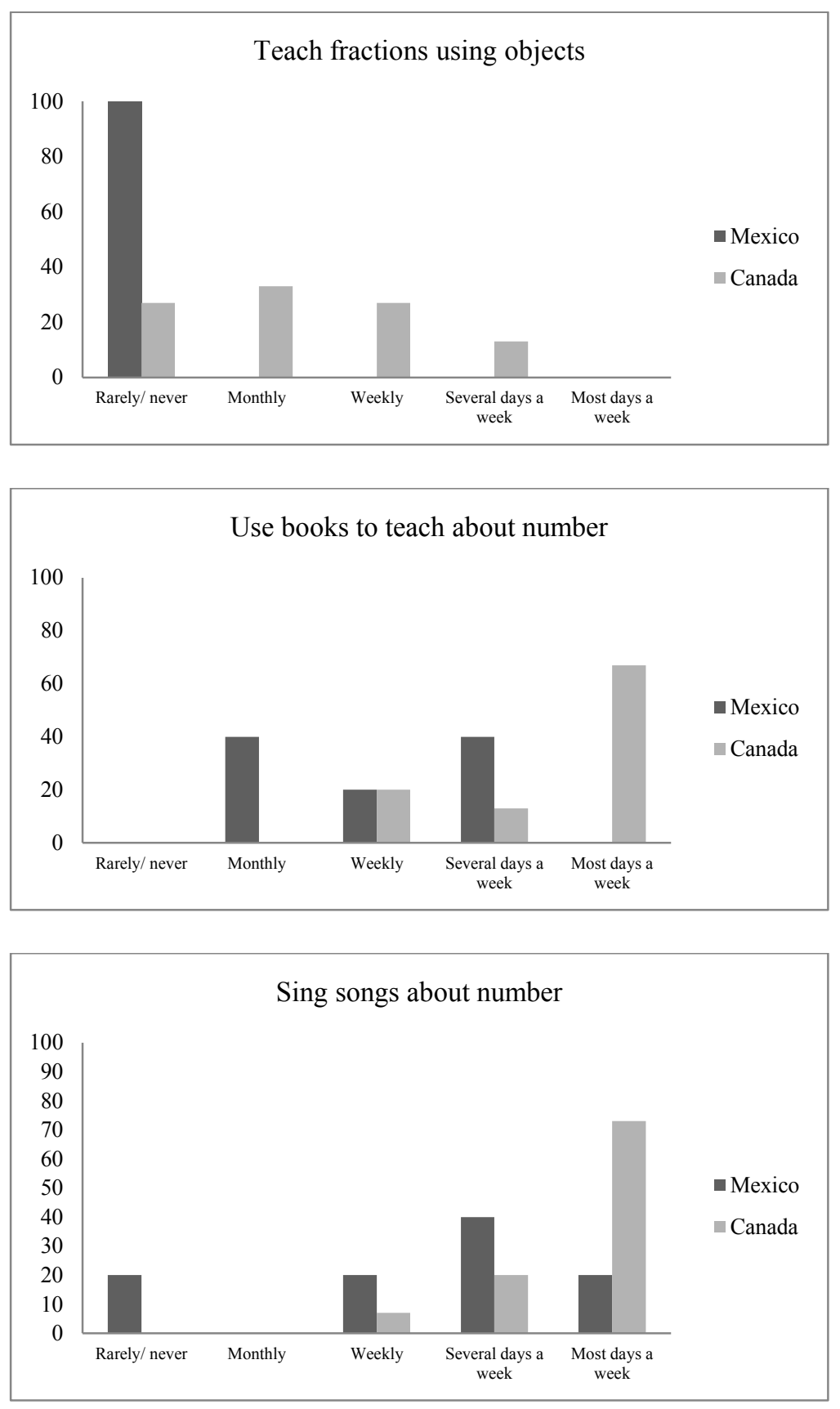
Figure 6.4. Distribution of responses for items on which Mexican teachers reported greater frequencies.
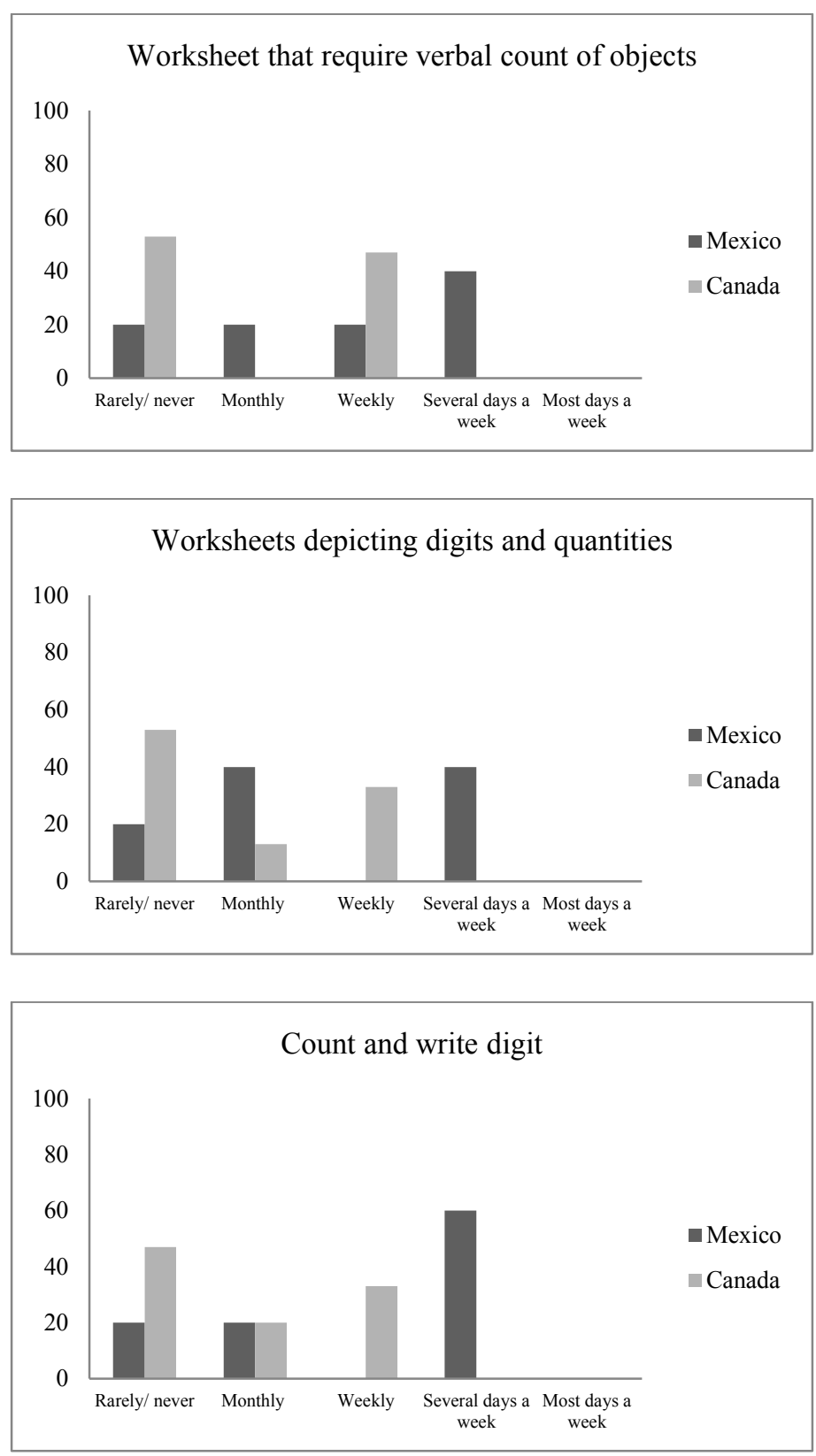
Responses from Canadian teachers also show a more informal approach to literacy, for example, all daycare teachers reported reading to the children, allowing time for children to read on their own, and asking questions when reading most days of the week (see Table 6.15). Singing the alphabet song was reported by all Canadian teachers as done more than once a week, in addition, more than $80 \%$ reported explaining new and unfamiliar words to the children most days of the week. Teachers in both countries showed a similar pattern of responses for teaching children to recognize printed letters and words, teaching children to print words, and Mexican teachers reported more frequently teaching children to print their names (Figure 6.5).

Figure 6.5. Distribution of teacher-reported frequencies of asking children to print their names.

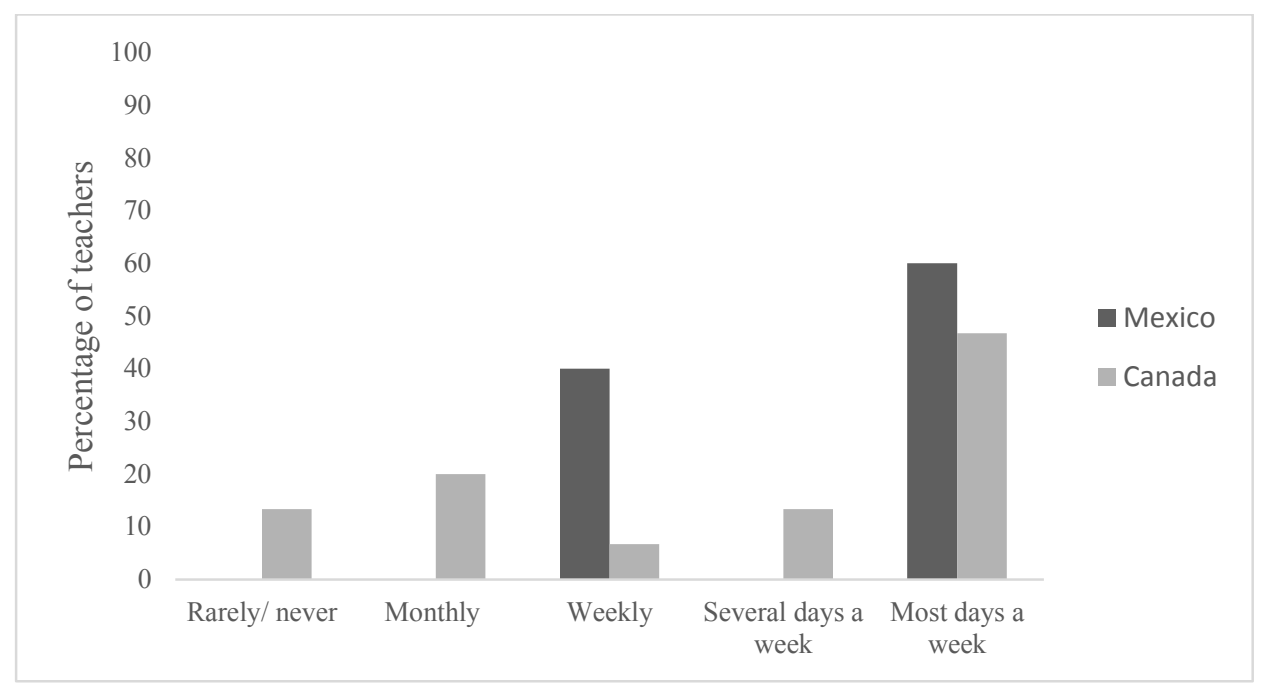


Table 6.14. Averaged Teachers' Reports of Classroom numeracy practice by country.

\begin{tabular}{|c|c|c|c|c|}
\hline \multirow[t]{2}{*}{ Items } & \multicolumn{2}{|c|}{ Mexico $(n=5)$} & \multicolumn{2}{|c|}{ Canada $(n=15)$} \\
\hline & $M$ & $S D$ & $M$ & $S D$ \\
\hline \multicolumn{5}{|l|}{ Word-digit } \\
\hline Show children the time while referring to the numbers on the clock & 2.20 & 2.05 & 3.07 & 0.92 \\
\hline Show children the date using the calendar or the board. & 3.60 & 0.55 & 3.00 & 1.41 \\
\hline Use books to teach the children about numbers (i.e., number recognition) & 2.00 & 1.00 & 3.47 & 0.83 \\
\hline Ask children to write the Arabic digits & 3.00 & 1.22 & 2.00 & 1.69 \\
\hline \multicolumn{5}{|l|}{ Word-quantity } \\
\hline Sing songs include number & 2.40 & 1.52 & 3.66 & 0.62 \\
\hline Engage children in activities that involve counting objects & 2.80 & 1.30 & 3.47 & 0.83 \\
\hline Ask children to use their fingers when counting & 3.00 & 1.22 & 2.93 & 1.44 \\
\hline Ask children to complete worksheets that require them to verbally count sets of objects & 1.80 & 1.30 & 0.93 & 1.03 \\
\hline \multicolumn{5}{|l|}{ Digit-quantity } \\
\hline Direct children's attention towards the digits and quantities on the wall & 1.20 & 1.30 & 2.67 & 1.40 \\
\hline Ask the children to complete worksheets that depict both the Arabic digits and the quantities & 1.60 & 1.34 & 0.80 & 0.94 \\
\hline Ask the children to count objects and then write the corresponding Arabic digit & 2.00 & 1.41 & 0.87 & 0.92 \\
\hline \multicolumn{5}{|l|}{ General Math } \\
\hline Teach the children simple sums/subtractions using objects & 0.80 & 1.10 & 1.73 & 1.44 \\
\hline Teach the children simple sums/subtractions using number words or Arabic digits & 0.40 & 0.89 & 1.20 & 1.61 \\
\hline Teach the children about fractions using objects (e.g., half an orange) & 0.00 & 0.00 & 1.27 & 1.03 \\
\hline Engage children in activities that require them to make measurements. & 0.40 & 0.55 & 1.40 & 0.99 \\
\hline Ask the children to classify objects by shape, color, and size. & 2.80 & 1.10 & 3.13 & 0.74 \\
\hline \multicolumn{5}{|l|}{ Comparison } \\
\hline Ask the children to compare sets of objects and identify which has a greater amount & 2.40 & 1.52 & 2.40 & 0.98 \\
\hline Ask children to compare number words and identify the one that represents a greater & 1.80 & 1.64 & 1.47 & 1.36 \\
\hline Ask the children to compare Arabic digits and tell you which represents a greater quant & 1.40 & 1.52 & 1.33 & 1.29 \\
\hline
\end{tabular}

Note. Teachers responded on a five-point scale to the question: How often do you do the following activities with the children?

Response options were 0- rarely or never, 1- monthly, 2- weekly, 3- several days a week, and 4- most days per week. 
Table 6.15. Averaged teachers' reports of literacy practice by country.

Items Mexico $(n=5) \quad$ Canada $(n=15)$

\begin{tabular}{llll}
\hline$M$ & $S D$ & $M$ & $S D$
\end{tabular}

Teach the children to recognize printed letters

$\begin{array}{llll}3.40 & 0.89 & 3.53 & 0.63 \\ 2.80 & 1.30 & 3.00 & 1.30 \\ 1.80 & 1.64 & 2.20 & 1.61 \\ 3.20 & 1.09 & 2.60 & 1.59 \\ 1.20 & 1.64 & 1.27 & 1.22 \\ 2.20 & 1.30 & 4.00 & 0 \\ 3.20 & 0.83 & 4.00 & 0 \\ 3.60 & 0.54 & 4.00 & 0 \\ 2.60 & 1.14 & 3.80 & 0.56 \\ 0.60 & 0.89 & 3.47 & 0.64\end{array}$

Sing the alphabet song

Note. Teachers responded on a five-point scale to the question: How often do you do the following activities with the children? Response options were 0- rarely or never, 1- monthly, 2- weekly, 3- several days a week, and 4- most days per week. 


\section{Summary}

The first goal of the present study was to examine whether there were differences between Canadian and Mexican parents' reports of academic expectations and frequency of engaging in home numeracy and literacy practices. There were significant differences with regards to parents' expectations. Mexican parents considered it important for children to achieve advanced numeracy and literacy skills such as counting to 1000 and read chapter books by the time they began Grade 1 . The high expectations likely reflect a poor understanding of the development of numeracy and literacy skills in young children.

Consistent with prior research (e.g., Kleemans et al., 2012; LeFevre, Polyzoi et al., 2010; Segers et al., 2015) in both countries parents who reported higher academic expectations for their children also reported engaging more frequently in formal advanced numeracy activities. There was no relationship between academic expectations and reported frequency of formal basic and mixed numeracy activities or with either formal advanced, basic, or mixed literacy activities. Maternal education was found to be unrelated to the reported frequency of engaging in home numeracy and literacy activities in both countries.

Parent reported frequency of engaging in formal advanced numeracy practices was marginally related to Canadian children's knowledge of the mapping between digits and number words. Furthermore, Canadian children's knowledge of cardinality skills were significantly related to the parent-reported frequency of engaging in formal basic numeracy practices. In contrast, parent reported frequency of numeracy and literacy practices were unrelated to Mexican children's performance on all the numeracy measures. 
Maternal education was found to be an important predictor of Canadian and Mexican children's numeracy skills. For the Mexican children, maternal education was related to the numeracy measures that involved knowledge of the verbal number words. For the Canadian children, maternal education predicted performance on the general numeracy measure, knowledge of the counting string and as with the Mexican children, knowledge of cardinality. Literacy practices were unrelated to performance on the math measures.

With regards to the daycare and preschool learning environment, some differences were observed in the amount of visual numerical stimuli that children are exposed to within their classrooms. Canadian classrooms had more books and more other resources such as clocks and calendars. These differences presumably reflect differences in the amount of economic resources available to the preschools and daycares. Importantly however, in classrooms in which the Arabic digits were displayed on the wall, more Canadian daycares displayed a larger range of numbers (i.e., 1-20) compared to the Mexican classrooms in which the highest number displayed was 10 .

Findings from the teacher questionnaire also indicated that different approaches were taken by daycare and preschool teachers. Canadian daycare teachers follow a more informal approach such that math activities are embedded in everyday activities and routines (e.g., circle and lunch time), children are read to every day, and allowed time to read daily. Mexican preschool teachers' responses are more consistent with a more direct and formal approach to teaching. 


\section{CHAPTER 7: GENERAL DISCUSSION}

The goals of the present research were to examine the mappings among quantities, words, and digits in two-to-four-year-old children, to explore the order in which these mappings are acquired, and to examine the relations between mapping, and other early numeracy skills such as verbal counting, cardinality, and symbolic number comparison skills. Building on prior models of early numeracy development by LeFevre, Fast. et al. (2010), and von Aster and Shalev (2007), I developed a model of early numeracy development in which the mapping skills were included as mediators of the relation between early quantitative and symbolic knowledge and symbolic number comparison. The model was tested with samples of Canadian and Mexican preschool children. Results from the present research suggest that children integrate their early quantitative and symbolic skills in order to develop the mappings between the three representations of number. Furthermore, the present findings are consistent with prior research in which children's knowledge of the underlying quantity represented by a number symbol is related to their ability to succeed on symbolic magnitude comparison tasks (Batchelor, Inglis, \& Gilmore, 2015). Thus, the present research supports the view that children's mapping skills are an important intermediate step between children's early symbol and quantity knowledge and the development of more complex math skills.

The present research also assessed the sequence of acquisition of the mappings between the three number representations. Bialystok (1992) proposed that children initially learn the mapping between number words and digits, then between number words and quantities; finally, the mapping between digits and quantities was proposed to develop once the other mappings were in place. Research on children's mapping skills 
has found inconsistent results with regards to the order of acquisition of the mappings. For example, Benoit et al. (2013) found that children initially develop the mapping between number words and quantities, then between digits and quantities, and finally between number words and digits. In contrast, Knudsen et al. (2015) found that children develop the mapping between digits and quantities after the mappings between number words digits and number words and quantities; however, the two mappings that involve digits were found to develop concurrently. Results from Studies 1 and 2 are consistent with the model proposed by Bialystok (1992); I found that in the three samples of children, performance was better on the number-word-digit mapping and worse on the digit-quantity mapping. In addition, the probability of success on the digit-quantity mapping tasks was greater if children were successful at mapping number words and digits and number words and quantities. Thus, the replication of the results in samples of children with different cultural and educational backgrounds suggest that the order of acquisition of the mappings may be a stable developmental progression (i.e., in the three samples more children performed better mapping digits-words, then words-quantities, and were least likely to succeed on digit-quantity mapping. The developmental trend replicates regardless of differences in educational or cultural experiences).

Despite the similarity in the order of acquisition of the mappings between Mexican and Canadian children, Mexican children showed lower performance on all the early numeracy measures than Canadian children. Researchers have found that differences in home numeracy practices are related to children's varying levels of performance (e.g., Huntsinger, 2000; LeFevre et al., 2002; LeFevre, Polyzoi et al., 2010). In the present research, however, parents' reports of the frequency of engaging in 
numeracy activities was only related to Canadian children's numeracy skills. For the Mexican children, parental expectations, reports of home numeracy and literacy were unrelated to the children's numeracy performance. The differences in the relations between the home learning environment and Mexican and Canadian children's performance on the numeracy assessments suggest that there may be cultural differences in the way in which early home education is conceived in different countries (e.g., Reese et al., 1995).

A limitation that should be considered when interpreting the results from the model proposed in the present research is that the sample sizes in Studies 1 and 2 were relatively small for path analyses and thus the results may not be generalizable. However, although further replication is warranted, the results from the Canadian children can be viewed with an acceptable degree of confidence given that a) the models in Studies 1 and 2 were theoretically motivated, and b) that the pattern of results found in Study 1 was replicated in Study 2. With regards to the model results for the Mexican children (Study 2), further research that includes larger samples of children from different socioeconomic backgrounds is required to understand whether the differences between the Mexican and Canadian children resulted from the sample size limitation.

\section{Precursor skills to mapping}

Several precursor skills were shown to predict children's mapping. Verbal counting was significantly related to children's cardinality and symbol knowledge, and, in Study 2, was related to children's digit-quantity mapping. Children's ability to understand and manipulate quantities (which requires understanding of cardinality for 
numbers beyond the subitizing range) was related to their word-quantity mapping in Study 1. Cardinality knowledge, predicted children's word-quantity mapping in Study 2. Researchers have found that children's advanced counting skills are predictive of later math performance (Aunola, Leskinen, Lerkkanen, \& Nurmi, 2004; Chu, van Marle, \& Geary, 2015; Manfra, Dinehart, \& Sembiante, 2014; Nguyen, Watts, Duncan, Clements, Sarama, Wolfe, \& Spitler, 2016; Tobia, Bonifacci, \& Marzocchi, 2015). The present research may provide an explanation for why counting skills are important, suggesting that they are related to children's more complex math abilities through their mapping skills. The relationship between counting, cardinality, and children's mapping skills was consistently found in the three groups of participants, replicating results from prior research (Batchelor, Kleeble, \& Gilmore, 2015; Lipton \& Spelke, 2005).

The finding that children's cardinality skills predicted their digit-word mapping in both Study 1 and 2 had not been predicted. This finding could reflect children's understanding of the number words as symbolic representations of quantity. Understanding the number words as representations of quantity is required in both cardinality and digit-word mapping tasks. Thus, children who understand the verbal number words as representations may find it easier to associate them with the corresponding written symbol (e.g., Neumann, Hood, Ford, \& Neumann, 2013).

Symbolic knowledge. Children's ability to identify number words and Arabic digits as a different category of symbols was hypothesized to be a precursor to children's word-digit mapping skills. Prior research by Mix (2009) had found that children begin to identify the written number symbols shortly after learning the count list and before they understand the cardinality principle. Study 1 showed that preschool children have the 
ability to recognize both the verbal and written number representations as distinct categories of symbols (i.e., numerical symbols) and can differentiate them from similar sounding words and letters that have similar shapes. Moreover, consistent with LeFevre, Fast. et al. (2010), children's symbolic identification skills were found to be unrelated to their quantity skills and as hypothesized, predictive of children's word-digit mapping skills.

\section{Children's mapping skills}

Order of acquisition of the mappings. In Study 1, Canadian children's knowledge of the mapping between number words and digits and number words and quantities predicted digit-quantity mapping. Although in Study 2 there were some differences between the results of the path analyses in the two samples (i.e., wordquantity mapping did not predict Mexican children's quantity-digit mapping skills) the overall pattern of results for both groups was consistent with the findings of Study 1. In addition, results from categorical analyses on both Canadian and Mexican children showed that the probability that children would succeed on the digit-quantity mapping is higher when children succeed on the word-digit and word-quantity mapping tasks. Brought together, the results from Studies 1 and 2 support the hypothesis that the mapping between digits and quantities develops later than the mappings between number words and digits and number words and quantities (Bialystok, 1992; Knudsen et al., 2015), and provides evidence that the developmental sequence of acquisition of the mappings among the number representations is a stable progression.

It is possible that the order in which children were observed to acquire the mappings is related to the way in which children are taught about number in Canada and 
Mexico. Alternate sequences of acquisition of the mappings might be observed if children learned the mappings between number words and quantities before learning the written number representations; in fact, Knudsen et al. (2015) found that children learned to map quantities to number words first and then simultaneously learned the mappings of digits to number words and digits to quantities. In the present research none of the Canadian children (in Studies 1 and 2) who failed to map words to digits were successful at mapping words to quantities. It is possible that children in Canada are exposed to the number symbols and begin learning their names through children's books and educational TV series at a very young age and before they are aware of the meaning of the symbols (see also Mix, 2009). The pattern of results was similar for the Mexican children in Study 2: of the 37 children who failed to map digits to words only three successfully mapped words to quantities. Mexican parents have been found to show preference for a more didactic approach to teaching their children (e.g., writing numbers and letters over and over until they can do it correctly) therefore this type of teaching may also foster children's acquisition of the mappings among the symbolic representations prior to understanding how they are related to the quantities they represent. Children could also potentially learn the mapping between digits and quantities prior to learning the mapping between number words and digits as found by Benoit et al (2013) although the process would be one of learning how a digit with no verbal label is related to a specific quantity and would almost necessarily be learnt from repeated simultaneous visual exposure to both the digit and the quantity.

Direction of mapping. Researchers have asked whether children's acquisition of mapping skills varies with the direction of mapping, for example, is it harder to map from 
digits to quantities than from quantities to digits? Mundy and Gilmore (2009) found an effect of direction when assessing children's symbol-quantity mapping whereas Brankaer et al. (2014) did not find a difference in performance on symbol-quantity mapping tasks as a function of direction of mapping. One possibility is that the effect of direction is a result of the size of the numbers used in the mapping tasks (Brankaer et al., 2014). For example, the mapping task used in the study by Mundy and Gilmore (2009) included numbers in the range of 20 to 50, whereas Brankaer et al. included only numbers from 19. In contrast, Odic et al. (2015) found a significant effect of direction of mapping using numbers in the range of 1-10. In Study 1 of the present research, I found that children performed better when mapping number words to quantities than the reverse whereas no effect of direction was found for the word-digit or for the digit-quantity mapping tasks. In Study 2 , there was no effect of direction on any of the six mapping tasks. The contrasting findings in the present research could be related to the order of presentation of the tasks. In Study 1, all the children received the same mapping direction (quantity-to-numberword) first, thus the possibility that the higher performance on the number-word-toquantity mapping could reflect a practice effect cannot be ruled out. In Study 2, the order in which the mapping tasks were presented varied across children. Overall, the evidence seems to indicate that children's mapping performance is equivalent in both directions.

\section{Symbolic number comparison.}

The present research further assessed the relationship between children's mapping and symbolic number comparison skills. The expected relationship between children's symbol-quantity mappings and symbolic number comparison skills was found in Studies 1 and 2 and, importantly, the strength of the relationship was the same in both Mexican 
and Canadian children (Study 2). Thus, results from the present studies are consistent with the proposal by Bialystok (1992) and with prior research in consistently showing that children's mapping skills predict performance on more advanced mathematical skills (e.g., Batchelor, Inglis, \& Gilmore, 2015; Brankaer et al., 2014; Mundy \& Gilmore, 2009). The present research suggests that the development of the mapping skills is a critical intermediate step that allows children to access quantity information from the number symbols. Direct access of quantity from the number symbols allows them to efficiently use and process the number symbols and supports their acquisition of later mathematical skills (e.g., Defever, DeSmedt, \& Reynvoet, 2013; De Smedt \& Gilmore, 2011; Gersten, Jordan, \& Flojo, 2005; Iuculano, Tang, Hall, \& Butterworth, 2008; Rousselle \& Noël, 2007).

\section{Children's numeracy performance and the home learning environment}

In Study 2, Mexican children's performance on all the numeracy assessments was significantly lower than that of Canadian children, even though the Mexican children were in a more formal instructional setting. Prior research has found that children's numeracy performance is significantly related to their home learning environment (e.g., Blevins-Knabe et al., 1996; Kleemans et al., 2012; LeFevre et al., 2002; LeFevre et al., 2009; LeFevre, Polyzoi et al., 2010; Manolitsis et al., 2013; Segers et al., 2015;

Skwarchuk, 2009; Skwarchuk et al., 2014). Furthermore, research has also found differences in the home learning environment associated with culture (Huntsinger et al., 2000; LeFevre, Polyzoi, et al., 2010; Starkey \& Klein, 2008), SES (DeFlorio et al., 2015), and parental education (e.g., Davis-Kean, 2005; Gonzalez, Acosta, Davis, PollardDurodola, Saenz, Soares, Resendez, \& Zhou, 2016; Harding et al., 2015). 
Consistent with prior research (e.g., Huntsinger et al., 2000; Kleemans et al., 2012; LeFevre, Polyzoi et al., 2010, Kleemans et al., 2012; Segers et al., 2015;

Skwarchuk, 2009; Skwarchuk et al., 2014), in the present research parents who reported having greater academic expectations also reported engaging more frequently in teaching their children early numeracy skills (e.g., teaching them to do simple sums). However, parents' reports of the frequency of home numeracy activities only predicted children's performance for Canadian, not for Mexican children. The pattern of parents' reports across cultures suggested that Mexican parents either did not have good knowledge of children's skills, or that they interpreted the questions differently than Canadian parents. For example, Mexican parents rated all of the numeracy benchmarks as more important for students entering Grade 1 than did the Canadian parents. The higher expectations were, in some cases, unrealistic. For example, Mexican parents considered it important for children to know multiplying and count to 1000, and read chapter books by the time they began Grade 1, suggesting that Mexican parents' poor knowledge of children's early numeracy and literacy development (e.g., DeFlorio \& Beliakoff, 2015).

With regards to numeracy practices, findings from the Canadian parents replicated past findings showing that parents engage more frequently in literacy than in numeracy activities (e.g., Blevins-Knabe et al., 2000; LeFevre et al., 2009). In contrast, Mexican parents reported engaging more frequently in numeracy than in literacy practices. Manolitsis et al. (2013) found a similar pattern of results in a study including Greek children and their parents; the authors associated the difference in the pattern of results to the fact that Greek is a more transparent orthography and thus, Greek parents may be 
aware that learning to read is relatively easy and consequently focus more on numeracy than on literacy practices.

Spanish is also a transparent orthography, and learning to read in Spanish has been found to be easier than learning to read in English (Aro \& Wimmer, 2003). However, in the present study, given Mexican parents' higher and unrealistic expectations, it is unlikely that the pattern of results reflects their knowledge that learning to read in Spanish is relatively easy. The pattern of results could however, reflect, the effect of parents' contact with their children's teacher. Preschool teachers are likely to be aware that learning to read is relatively easy, and may thus encourage parents to focus more on numeracy activities at home.

Another explanation for the differences between Canadian and Mexican results could be that the parent questionnaire that has been used to assess home practices in Canadian homes may not have been suitable for the Mexican parents because of the cultural differences in the approach to early education between the countries. For example, although storybook reading is a frequently reported practice among Canadian parents (e.g., Skwarchuk et al., 2014), story telling was found to be a frequently reported practice in Mexican homes (Reese, 2012). Furthermore, story-telling was not done exclusively by the parents, but by members of the extended family and was a frequent activity that children were engaged in during family reunions, for example, telling of horror stories, family anecdotes, and jokes.

Another potential cultural difference that was not captured in the present study was that research conducted within the USA with immigrant Latino parents showed that when they engaged in teaching their children literacy, they mainly used a formal didactic 
approach based on memory and repetition (e.g., writing letters or reading words over and over until the child learns it by heart); these activities were rarely embedded in the context of their everyday lives and routines (Goldenberg, Reese, \& Gallimore, 1992). Thus, it is possible that the questionnaire could have omitted other culture-specific literacy or numeracy practices.

With regards to the relationship between performance on numeracy assessments and parent-reported frequency of engaging in home numeracy and literacy practices, consistent with prior research (e.g., LeFevre et al., 2002) results from the Canadian children and parents showed a significant relationship between children's cardinality skill and reported frequency of basic numeracy practices, and a marginal relation between children's knowledge of the digit-word mapping and parents' reported frequency of formal advanced numeracy activities. In contrast, Mexican children's performance was unrelated to parents' reports of home numeracy and literacy practices. This last finding could reflect that parents have only recently begun teaching their children numeracy and literacy skills (e.g., Reese \& Gallimore, 2000) and that the effects of the teaching are not yet apparent. Alternatively, it may reflect social-desirability biases in Mexican parents' answers to the questionnaire. Preliminary work with children from a similar Spanishspeaking culture in Chile (Susperreguy, Jiménez Lira, \& LeFevre, in preparation) similarly did not show a relation between parents' reports and children's numeracy skills. Further research is needed to understand these cultural differences.

\section{Preschool and daycare learning environment}

There were some differences with regards to the preschool learning environment in Canada and Mexico. Compared to Mexican preschool classrooms, a greater percentage 
of Canadian daycare classrooms displayed numbers (e.g., clocks, calendars, written on the walls), and had number books. Furthermore, when the numbers were displayed on the walls, the range of numbers was 1-20 as opposed to 1-10 in the Mexican classrooms. Teachers' approach to learning also differed across countries. Canadian daycare teachers are expected to follow an informal, play-based approach to learning. In contrast, Mexican preschool teachers are expected to formally teach children numeracy and literacy skills. Teachers' responses to the questionnaire reflected the differences in the approach to teaching children. For example, Canadian teachers reported singing the alphabet and songs about numbers, using books to teach the children about number more frequently than Mexican teachers. In contrast, Mexican teachers reported more frequently engaging the children in completing worksheets and counting and writing the numbers than the Canadian teachers.

The different approaches to teaching may have affected children's numeracy skills, for example, because Canadian children are exposed to math activities during their daily routines (e.g., circle time) they may be more likely to carry out similar activities (e.g., singing the counting song, counting cups during meal times) by themselves during the course of the day. In contrast, if Mexican children are exposed to activities that are difficult to generalize (e.g., completing worksheets), it may be difficult for them to translate the acquired knowledge to other situations during their day (e.g., they may not see how they can use number to determine the number of cups needed for everyone at the table). 


\section{Limitations}

The present research had several limitations. First, the sample sizes in Studies 1 and 2 were relatively small. Replication of the proposed model in larger samples is needed if the results are to be generalized. In Study 2, the small number of preschools and daycares that participated limited our ability to statistically assess the relationship between children's performance and teachers' reports of the frequency of numeracy and literacy practices. In addition, Mexican children in Study 2 were recruited from public preschools serving mainly middle- to lower-SES families, thus, the results may not extend to children who attend private schools and who come from higher SES families.

A second limitation of the present research was the cross sectional design, which provides only an initial insight into the developmental sequence of acquisition of the mappings. Longitudinal studies are needed to assess the developmental sequence of acquisition of the mappings depicted in the model proposed in Study 1.

Longitudinal studies are also important in evaluating the sources of the observed differences in early numeracy skills between the Canadian and Mexican children. Differences in children's numeracy experiences prior to school may be an important source of these differences, a possibility that is consistent with the findings that children in many cultures arrive at school with widely varying levels of early numeracy skill (e.g., Starkey \& Klein, 2008). Mexican parents may not engage in teaching their children academic skills until they enter school (e.g., Reese \& Gallimore, 2000) whereas Canadian parents may engage their children in numeracy activities from an early age. If such difference exist, Mexican children would have been exposed to home numeracy activities only for the time they had been in school (i.e., eight months), explaining their relatively low performance, compared to longer for Canadian children. Thus, longitudinal studies 
would be extremely helpful in explaining the differences in numeracy performance between Canadian and Mexican children encountered in the present research.

A third limitation, and potential source of some of the differences across the groups, is that the context in which parents answered the questionnaire differed in Canada and Mexico. In Canada, questionnaires were given to the parents to complete individually at home, whereas parents in Mexico responded to the questionnaire during a meeting in which the preschool director, their children's teacher, and the experimenter were present. This form of collecting parent data could have led them to respond to the questions in a socially desirable way.

A related limitation is that the questionnaire used to assess the home learning environment may not have included home learning activities that are culture-specific. For example, oral story telling is more common in Mexican homes than shared reading (Reese, 2012). A more suitable instrument to assess the home learning environment might need to include assessment of oral story-telling and other literacy and numeracy practices not captured in the questionnaire. In depth interviews and observations of interactions between parents and children in Mexican homes are necessary in order to ensure that suitable instruments are used to assess the home numeracy environment.

A limitation of Study 3 was that Canadian children who participated in the intervention already had sufficient understanding of cardinality such that there was no room for improvement with training. In essence, it was not possible to determine whether the training procedure itself was ineffective or whether the intervention had no effect because the targeted skills in the children were already well developed. 


\section{Conclusions}

The present research provides several contributions to the literature on children's mapping skills (e.g., Batchelor, Inglis, \& Gilmore, 2015; Brankaer et al., 2014; Benoit et al., 2013; Knudsen et al., 2015; Mundy \& Gilmore, 2009). First, I simultaneously assessed of mappings among the three representations in two studies using three different samples, and using tasks that were designed to be equated for difficulty and task demands. Thus, the present research is a more thorough exploration of children's mapping performance than is found in existing research. Second, none of the studies of children's mapping included a model of early numeracy development or examined the relations between children's precursor, mapping, and symbolic number comparison skills (cf. Batchelor, Inglis, \& Gilmore, 2015; Batchelor, Keeble, \& Gilmore, 2015; Brankaer et al., 2014; Lipton \& Spelke, 2005; Mundy \& Gilmore, 2009; Odic et al., 2015). The present research has thus made a valuable contribution to the field by expanding the Pathways Model (LeFevre, Fast et al., 2010) with the inclusion of children's mapping skills. As in the Pathways model, children's early skills could be grouped into distinct linguistic and quantitative precursor pathways. The present research suggests that the integration of the linguistic/symbolic and quantitative precursor pathways occurs as children acquire digit-quantity mapping skills. Furthermore, this research expands our understanding of how early precursor skills such as verbal counting and symbol knowledge are linked through the mapping performance to symbolic comparison. Symbolic (specifically digit comparison) tasks are strong predictors of children's arithmetic skills in kindergarten and the early grades (De Smedt \& Gilmore, 2011; De Smedt et al., 2013; Holloway \& Ansari, 2009; Lyons et al., 2014; Vanbinst \& De Smedt, 
2016; Vanbinst, Ghesquière, \& DeSmedt, 2015) and recently have been claimed to be as important to mathematical development as phonological awareness is to reading (Vanbinst, et al., 2016).

Another contribution of the present research is that it provided evidence to suggest that the sequence of acquisition of the mappings is stable. Prior studies had not attempted to compare mapping skills in children from different cultural and educational backgrounds. In the present studies, the pattern of results from Canadian and Mexican children showed many similarities, suggesting that the proposed sequence of acquisition of the mappings and the relationships between children's precursor, mapping, and symbolic number comparison skills follow a generally stable progression. Past research had found contrasting results with regards to the acquisition of the mappings (e.g., Benoit et al., 2013; Knudsen et al., 2015), however, the replication in Study 2 of the results from the analyses of the relationships between the mapping tasks suggests children initially develop the mappings between number words and digits, then between number words and quantities, and that these two mappings allow children to develop the association between digits and quantities. The present research has thus provided insight into the developmental sequence of acquisition of the mappings in very young children.

A further contribution of the present study was the examination of the home numeracy environment in Canadian and Mexican homes. Although much research has been conducted on the early home learning environment in Canada and the United States (e.g., Blevins-Knabe et al., 1996; Blevins-Knabe et al., 2000; Huntsinger et al., 2000; LeFevre et al 2002; LeFevre et al., 2009; LeFevre, Polyzoi et al., 2010; Skwarchuk, 2009; Skwarchuk et al., 2014) and Europe (Anders et al., 2012; Kleemans et al., 2012; 
Manolitsis et al., 2013; Melhuish et al., 2008; Segers et al., 2015) the present study is the first to assess the home learning and its relation to children's early numeracy skills in Mexico. Findings from the present study provide some insight as to the possible reasons behind the lower math skills exhibited by the Mexican students on international assessments of math such as the Programme for International Student Assessment (PISA) exam. The PISA exam assesses 15-year-olds' competencies in science, math, and reading. According to the PISA results for 2012, 55\% of Mexican 15-year-olds failed to achieve basic math competencies compared to $14 \%$ of Canadian 15 -year-olds (OECD, 2012). Thus, longitudinal studies would be extremely helpful in explaining Mexican children's lower numeracy performance and the possible consequences associated with the later acquisition of early numeracy skills. 


\section{REFERENCES}

Agrillo, C., Piffer, L., Bisazza, A., \& Butterworth, B. (2012). Evidence for two numerical systems that are similar in humans and guppies. PloS One, 7(2), e31923. doi:10.1371/journal.pone.0031923

Ansari, D. (2008). Effects of development and enculturation on number representation in the brain. Nature Reviews Neuroscience, 9, 278-91.

Anders, Y., Rossbach, H. G., Weinert, S., Ebert, S., Kuger, S., Lehrl, S., \& von Maurice, J. (2012). Home and preschool learning environments and their relations to the development of early numeracy skills. Early Childhood Research Quarterly, $27(2), 231-244$.

Aro, M., \& Wimmer, H. (2003). Learning to read: English in comparison to six more regular orthographies. Applied psycholinguistics, 24(04), 621-635.

Aunola, K., Leskinen, E., Lerkkanen, M. K., \& Nurmi, J. E. (2004). Developmental dynamics of math performance from preschool to grade 2. Journal of Educational Psychology, 96(4), 699.

Batchelor, S., Inglis, M., \& Gilmore, C. (2015). Spontaneous focusing on numerosity and the arithmetic advantage. Learning and Instruction, 40, 79-88.

Batchelor, S., Keeble, S., \& Gilmore, C. (2015). Magnitude representations and counting skills in preschool children. Mathematical Thinking and Learning, 17(2-3), 116135. doi:10.1080/10986065.2015.1016811

Benoit, L., Lehalle, H., Molina, M., Tijus, C., \& Jouen, F. (2013). Young children's mapping between arrays, number words, and digits. Cognition, 129, 95-101. doi:10.1016/j.cognition.2013.06.005. 
Bialystok, E. (1992). Symbolic representation of letters and numbers. Cognitive Development, 7, 301-316.

Bialystok, E., \& Codd, J. (1996). Developing representations of quantity. Canadian Journal of Behavioural Science, 28, 281-291.

Bialystok, E., \& Codd, J. (2000). Representing quantity beyond whole numbers: some, none, and part. Canadian Journal of Experimental Psychology, 54, 117-128.

Blevins-Knabe, B. (2008). Fostering early numeracy at home. Encyclopedia of Language and Literacy Research.

Blevins-Knabe, B., Austin, A. B., Musun, L., Eddy, A., \& Jones, R. M. (2000). Family Home Care Providers' and Parents’ Beliefs and Practices Concerning Mathematics with Young Children. Early Child Development and Care, 165(1), 41-58.

Blevins-Knabe, B., \& Musun-Miller, L. (1996). Number use at home by children and their parents and its relationship to early mathematical performance. Early Development and Parenting, 5(1), 35-45.

Brankaer, C., Ghesquière, P., \& De Smedt, B. (2014). Children's mapping between nonsymbolic and symbolic numerical magnitudes and its association with timed and untimed tests of mathematics achievement. PloS one, 9(4), e93565.

Boonen, A. J., Kolkman, M. E., \& Kroesbergen, E. H. (2011). The relation between teachers' math talk and the acquisition of number sense within kindergarten classrooms. Journal of School Psychology, 49(3), 281-299.

Carey, S. (2004). Bootstrapping and the origin of concepts. Daedalus, 59-68. 
Castronovo, J., \& Göbel, S. M. (2012). Impact of high mathematics education on the number sense. PloS One, 7(4), e33832. doi:10.1371/journal.pone.0033832

Condry, K. F., \& Spelke, E. (2008). The development of language and abstract concepts: The case of natural number. Journal of Experimental Psychology, 137, 22-38. doi: $10.1037 / 0096-3445.137 .1 .22$

Connolly, A. J. (2000). KeyMath-revised/updated Canadian norms. Richmond Hill, Ontario: PsyCan.

Chu, F.W., vanMarle, K., \& Geary, D.C. (2013). Quantitative deficits of preschool children at risk for mathematical learning disability. Frontiers in Psychology, 4, $1-10$.

Davis-Kean, P. E. (2005). The influence of parent education and family income on child achievement: the indirect role of parental expectations and the home environment. Journal of family psychology, 19(2), 294.

Defever, E., De Smedt, B., \& Reynvoet, B. (2013). Numerical matching judgments in children with mathematical learning disabilities. Research in developmental disabilities, 34(10), 3182-3189.

DeFlorio, L., \& Beliakoff, A. (2015). Socioeconomic Status and Preschoolers' Mathematical Knowledge: The Contribution of Home Activities and Parent Beliefs. Early Education and Development, 26(3), 319-341.

De Smedt, B., \& Gilmore, C. K. (2011). Defective number module or impaired access? Numerical magnitude processing in first graders with mathematical difficulties. Journal of experimental child psychology, 108(2), 278-292.

De Smedt, B., Noël, M.-P., Gilmore, C., \& Ansari, D. (2013). How do symbolic and non- 
symbolic numerical magnitude processing skills relate to individual differences in children's mathematical skills? A review of evidence from brain and behavior. Trends in Neuroscience and Education. 2, 48-55.

De Smedt, B., Verschaffel, L., Ghesquiere, P. (2009). The predictive value of numerical magnitude comparison for individual differences in mathematics achievement. Journal of Experimental Child Psychology, 103, 469-479.

Durand, M., Hulme, C., Larkin, R., \& Snowling, M. (2005). The cognitive foundations of reading and arithmetic skills in 7-to 10-year-olds. Journal of Experimental Child Psychology, 91(2), 113-136.

Dyson, N. I., Jordan, N. C., \& Glutting, J. (2013). A number sense intervention for lowincome kindergartners at risk for mathematics difficulties. Journal of Learning Disabilities, 46(2), 166-181.

Feigenson, L., Dehaene, S., \& Spelke, E. (2004). Core systems of number. Trends in cognitive sciences, 8(7), 307-314.

Fuson, K. (1988). Children's counting and concepts of number. New York: SpringerVerlag.

Fuchs, L. S., Geary, D. C., Compton, D. L., Fuchs, D., Hamlett, C. L., Seethaler, P. M., ... \& Schatschneider, C. (2010). Do different types of school mathematics development depend on different constellations of numerical versus general cognitive abilities? Developmental psychology, 46(6), 1731.

Geary, D. C., Hoard, M. K., Nugent, L., \& Bailey, D. H. (2012). Mathematical cognition deficits in children with learning disabilities and persistent low achievement: A five-year prospective study. Journal of Educational Psychology, 104(1), 206. 
Geary, D. C., Bailey, D. H., \& Hoard, M. K. (2009). Predicting mathematical achievement and mathematical learning disability with a simple screening tool the number sets test. Journal of Psychoeducational Assessment, 27(3), 265-279.

Gelman, R. \& Gallistel, C.R. (1978). The Child's Understanding of Number. Harvard University Press.

Gersten, R., Jordan, N. C., \& Flojo, J. R. (2005). Early identification and interventions for students with mathematics difficulties. Journal of learning disabilities, 38(4), 293-304.

Gonzalez, J. E., Acosta, S., Davis, H., Pollard-Durodola, S., Saenz, L., Soares, D., ... \& Zhu, L. (2016). Latino Maternal Literacy Beliefs and Practices Mediating Socioeconomic Status and Maternal Education Effects in Predicting Child Receptive Vocabulary. Early Education and Development, 1-18.

Goldenberg, C., Reese, L., \& Gallimore, R. (1992). Effects of literacy materials from school on Latino children's home experiences and early reading achievement. American journal of Education, 497-536.

Gonzalez Estudillo, A. (2013). A latent growth curve analysis examining maternal education and parenting during childhood as predictors of academic achievement during the transition to adolescence (Doctoral dissertation, INDIANA UNIVERSITY).

Gunderson, E. A., \& Levine, S. C. (2011). Some types of parent number talk count more than others: relations between parents' input and children's cardinal-number knowledge. Developmental science, 14(5), 1021-1032. 
Harding, J. F., Morris, P. A., \& Hughes, D. (2015). The relationship between maternal education and children's academic outcomes: A theoretical framework. Journal of Marriage and Family, 77(1), 60-76.

Holloway, I.D., \& Ansari, D. (2008). Domain-specific and domain-general changes in children's development of number comparison. Developmental Science, 11, 644649.

Holloway, I.D., \& Ansari, D. (2009). Mapping numerical magnitudes onto symbols: The numerical distance effect and individual differences in children's mathematics achievement. Journal of Experimental Child Psychology, 103, 17-29.

Huntsinger, C. S., Jose, P. E., Larson, S. L., Balsink Krieg, D., \& Shaligram, C. (2000). Mathematics, vocabulary, and reading development in Chinese American and European American children over the primary school years. Journal of Educational Psychology, 92(4), 745.

Iuculano, T., Tang, J., Hall, C. W., \& Butterworth, B. (2008). Core information processing deficits in developmental dyscalculia and low numeracy.

Developmental science, 11(5), 669-680.

Jordan, N. C., Dyson, N., \& Glutting, J. (2011). Developing Number Sense in Kindergartners at Risk for Learning Difficulties in Mathematics. Society for Research on Educational Effectiveness.

Jordan, N. C., Glutting, J., Dyson, N., Hassinger-Das, B., \& Irwin, C. (2012). Building kindergartners' number sense: A randomized controlled study. Journal of Educational Psychology, 104(3), 647. 
Jordan, N. C., Glutting, J., \& Ramineni, C. (2010). The importance of number sense to mathematics achievement in first and third grades. Learning and individual differences, 20(2), 82-88.

Jung, S., Fuller, B., \& Galindo, C. (2012). Family functioning and early learning practices in immigrant homes. Child Development, 83(5), 1510-1526.

Kleemans, T., Peeters, M., Segers, E., \& Verhoeven, L. (2012). Child and home predictors of early numeracy skills in kindergarten. Early Childhood Research Quarterly, 27(3), 471-477.

Klibanoff, R. S., Levine, S. C., Huttenlocher, J., Vasilyeva, M., \& Hedges, L. V. (2006). Preschool children's mathematical knowledge: The effect of teacher" math talk.". Developmental Psychology, 42(1), 59.

Knudsen, B., Fischer, M.H., Henning, A., \& Aschersleben, G. (2015). The development of Arabic digit knowledge in 4-to-7-year-old children. Journal of Numerical Cognition, 1, 21-37.

Kolkman, M.E., Kroesbergen, E.H., \& Leseman, P.P.M. (2013). Early numerical development and the role of non-symbolic and symbolic skills. Learning and Instruction, 25, 95-103. doi: 10.1016/j.learninstruc.2012.12.001.

Koury, A. S., \& Votruba-Drzal, E. (2014). School readiness of children from immigrant families: Contributions of region of origin, home, and childcare. Journal of Educational Psychology, 106(1), 268. 
Krohn, K. R., Skinner, C. H., Fuller, E. J., \& Greear, C. (2012). Using taped intervention to improve kindergarten students' number identification. Journal of applied behavior analysis, 45(2), 437-441.

Krajewski, K., \& Schneider, W. (2009). Early development of quantity to number-word linkage as a precursor of mathematical school achievement and mathematical difficulties: Findings from a four-year longitudinal study. Learning and Instruction, 19, 513-526. doi: 10.1016/j.learninstruc.2008.10.002.

Landerl, K. (2013). Development of numerical processing in children with typical and dyscalculic arithmetic skills-a longitudinal study. Frontiers in Psychology, 4(July). doi:10.3389/fpsyg.2013.00459

Lareau, A. (2011). Unequal childhoods: Class, race, and family life. Univ of California Press.

LeCorre, M. \& Carey, S. (2007). One, two, three, four, nothing more: An investigation of the verbal counting principles. Cognition, 105, 395-438. doi:10.1016/j.cognition.2006.10.005.

Le Corre, M., Van de Walle, G., Brannon, E.M., \& Carey, S. (2006). Re-visiting the competence/performance debate in the acquisition of the counting principles. Cognitive Psychology, 52, 130-169. doi: 10.1016/cogpsych.2005.07.002.

LeFevre, J.-A., Clarke, T., \& Stringer, A. P. (2002). Influences of language and parental involvement on the development of counting skills: Comparisons of French-and English-speaking Canadian children. Early Child Development and Care, 172(3), 283-300. 
LeFevre, J.-A., Fast, L., Skwarchuk, S.-L., Smith-Chant, B.L., Bisanz, J., Kamawar, D., $\&$

Penner-Wilger, M. (2010). Pathways to mathematics: Longitudinal predictors of performance. Child Development, 81, 1753-1767.

LeFevre, J.-A., Polyzoi, E., Skwarchuk, S. L., Fast, L., \& Sowinski, C. (2010). Do home numeracy and literacy practices of Greek and Canadian parents predict the numeracy skills of kindergarten children? International Journal of Early Years Education, 18(1), 55-70.

LeFevre, J.-A., Skwarchuk, S. L., Smith-Chant, B. L., Fast, L., Kamawar, D., \& Bisanz, J. (2009). Home numeracy experiences and children's math performance in the early school years. Canadian Journal of Behavioural Science/Revue canadienne des sciences du comportement, 41(2), 55.

Levine, S.C., Jordan, N.C., \& Huttenlocher, J. (1992). Development of calculation abilities in young children. Journal of Experimental Child Psychology, 53, 72103.

Levine, S. C., Suriyakham, L. W., Rowe, M. L., Huttenlocher, J., \& Gunderson, E. A. (2010). What counts in the development of young children's number knowledge? Developmental psychology, 46(5), 1309.

Lipton, J. S., \& Spelke, E. S. (2005). Preschool children's mapping of number words to nonsymbolic numerosities. Child development, 76(5), 978-988.

Lyons, I.M., \& Beilock, S.L. (2011). Numerical ordering ability mediates the relation between number-sense and arithmetic competence. Cognition,121, 256-261. doi:10.1016/j.cognition.2011.07.009. 
Lopez, E. M., Gallimore, R., Garnier, H., \& Reese, L. (2007). Preschool Antecedents of Mathematics Achievement of Latinos The Influence of Family Resources, Early Literacy Experiences, and Preschool Attendance. Hispanic Journal of Behavioral Sciences, 29(4), 456-471.

Lyons, I.M., Price, G.R., Vaessen, A., Blomert, L., \& Ansari, D. (2014). Numerical predictors of arithmetic success in grades 1-6. Developmental Science, 1-13.

Mandler, G., \& Shebo, B. J. (1982). Subitizing: An analysis of its component processes. Journal of Experimental Psychology: General, 111(1), 1-22.

Manfra, L., Dinehart, L. H., \& Sembiante, S. F. (2014). Associations between counting ability in preschool and mathematic performance in first grade among a sample of ethnically diverse, low-income children. Journal of Research in Childhood Education, 28(1), 101-114.

Manolitsis, G., Georgiou, G. K., \& Tziraki, N. (2013). Examining the effects of home literacy and numeracy environment on early reading and math acquisition. Early Childhood Research Quarterly, 28(4), 692-703.

Melhuish, E. C., Phan, M. B., Sylva, K., Sammons, P., Siraj-Blatchford, I., \& Taggart, B. (2008). Effects of the home learning environment and preschool center experience upon literacy and numeracy development in early primary school. Journal of Social Issues, 64(1), 95-114.

Mix, K.S. (2002). The construction of number concepts. Cognitive Development, 17, $1345-1363$. 
Mix, K. S., Sandhofer, C. M., Moore, J. A., \& Russell, C. (2012). Acquisition of the cardinal word principle: The role of input. Early Childhood Research Quarterly, 27(2), 274-283.

Mix, K.S. (2009). How Spencer made number: First uses of the number words. Journal of Experimental Child Psychology, 102, 427-444.

Moll, K., Snowling, M. J., Göbel, S. M., \& Hulme, C. (2015). Early language and executive skills predict variations in number and arithmetic skills in children at family-risk of dyslexia and typically developing controls. Learning and Instruction, 38, 53-62. doi:10.1016/j.learninstruc.2015.03.004

Mundy, E., \& Gilmore, C. K. (2009). Children's mapping between symbolic and nonsymbolic representations of number. Journal of experimental child psychology, 103(4), 490-502.

Muthén, L.K., \& Muthén, B.O. (1998-2015). MPlus 7.31 [Computer software]. Los Angeles, CA: Muthén \& Muthén. http://statmodel.com.

Negen, J., \& Sarnecka, B. W. (2009). Young children's number-word knowledge predicts their performance on a non-linguistic number task. In N.A. Taatgen \& H. van Rijn (Eds.), Proceedings of the $31^{\text {st }}$ Annual Conference of the Cognitive Science Society (pp.2998-3003). Austin, Tx: Cognitive Science Society.

Neumann, M. M., Hood, M., Ford, R. M., \& Neumann, D. L. (2013). Letter and numeral identification: their relationship with early literacy and numeracy skills. European Early Childhood Education Research Journal, 21(4), 489-501.

Ng, F. F. Y., Tamis-LeMonda, C., Yoshikawa, H., \& Sze, I. N. L. (2015). Inhibitory control in preschool predicts early math skills in first grade Evidence from an 
ethnically diverse sample. International Journal of Behavioral Development, 39(2), 139-149.

Nguyen, T., Watts, T. W., Duncan, G. J., Clements, D. H., Sarama, J. S., Wolfe, C., \& Spitler, M. E. (2016). Which preschool mathematics competencies are most predictive of fifth grade achievement?. Early childhood research quarterly, 36, $550-560$.

Noël, M.-P., \& Rousselle, L. (2011). Developmental Changes in the Profiles of Dyscalculia: An Explanation Based on a Double Exact-and-Approximate Number Representation Model. Frontiers in Human Neuroscience, 5(December), 165. doi:10.3389/fnhum.2011.00165

Odic, D., Le Corre, M., \& Halberda, J. (2015). Children's mappings between number words and the approximate number system. Cognition, 138, 102-121. doi:10.1016/j.cognition.2015.01.008

OECD (2012). PISA Results in Focus, what 15-year-olds know and what they can do with what they know. Retrieved from: https://www.oecd.org/pisa/keyfindings/pisa2012-results-overview.pdf

Ontario Ministry of Education (2014). How does learning happen? Ontario's pedagogy from the early years. Retrieved from http://www.edu.gov.on.ca/childcare/HowLearningHappens.pdf.

Pan, Y., Gauvain, M., Liu, Z., \& Cheng, L. (2006). American and Chinese parental involvement in young children's mathematics learning. Cognitive Development, 21(1), 17-35. 
Piazza, M. (2010). Neurocognitive start-up tools for symbolic number representations. Trends in cognitive sciences, 14(12), 542-551.

Purpura, D.J., Baroody, A.J., \& Lonigan, C.J. (2013). The transition from informal to formal mathematical knowledge: Mediation by numeral knowledge. Journal of Educational Psychology, 1-12. doi: 10.1037/a0031753.

Purpura, D. J., Hume, L. E., Sims, D. M., \& Lonigan, C. J. (2011). Early literacy and early numeracy: the value of including early literacy skills in the prediction of numeracy development. Journal of Experimental Child Psychology, 110(4), 64758. doi:10.1016/j.jecp.2011.07.004

Purpura, D. J., \& Reid, E. E. (2016). Mathematics and language: individual and group differences in mathematical language skills in young children. Early Childhood Research Quarterly, 36, 259-268.

Ramani, G. B., Siegler, R. S., \& Hitti, A. (2012). Taking it to the classroom: Number board games as a small group learning activity. Journal of Educational Psychology, 104(3), 661.

Ramani, G. B., Rowe, M. L., Eason, S. H., \& Leech, K. A. (2015). Math talk during informal learning activities in Head Start families. Cognitive Development, 35 , $15-33$.

Range, F., Jenikejew, J., Schröder, I., \& Virányi, Z. (2014). Difference in quantity discrimination in dogs and wolves. Frontiers in Psychology, 5(November), 1-10. doi:10.3389/fpsyg.2014.01299

Reese, L. (2012). Storytelling in Mexican homes: Connections between oral and literacy practices. Bilingual research journal, 35(3), 277-293. 
Reese, L., \& Gallimore, R. (2000). Immigrant Latinos' cultural model of literacy development: An evolving perspective on home-school discontinuities. American Journal of Education, 103-134.

Reese, L., Balzano, S., Gallimore, R., \& Goldenberg, C. (1995). The concept of educación: Latino family values and American schooling. International Journal of Educational Research, 23(1), 57-81.

Rousselle, L., \& Noël, M.-P. (2007). Basic numerical skills in children with mathematics learning disabilities: a comparison of symbolic vs non-symbolic number magnitude processing. Cognition, 102(3), 361-95. doi:10.1016/j.cognition.2006.01.005

Sarnecka, B.W., \& Carey, S. (2008). How counting represents number: What children must learn and when they learn it. Cognition, 108, 662-674. doi:10.1016/j.cognition.2008.05.007.

Segers, E., Kleemans, T., \& Verhoeven, L. (2015). Role of Parent Literacy and Numeracy Expectations and Activities in Predicting Early Numeracy Skills. Mathematical Thinking and Learning, 17(2-3), 219-236.

SEP (2011). Programa de Estudio 2011, Guia para la Educadora. Retrieved from: http://www.reformapreescolar.sep.gob.mx/ACTUALIZACION/PROGRAMA/Pre escolar2011.pdf

Shusterman, A., Gibson, D., \& Finder, B. (2010). Acquiring first number words: The developmental trajectory of children's meanings for "Two." Proceedings of the 2009 Boston University Conference on Language Development, Cascadilla Press. 
Skwarchuk, S. L. (2009). How do parents support preschoolers' numeracy learning experiences at home? Early Childhood Education Journal, 37(3), 189-197.

Skwarchuk, S. L., Sowinski, C., \& LeFevre, J. A. (2014). Formal and informal home learning activities in relation to children's early numeracy and literacy skills: The development of a home numeracy model. Journal of experimental child psychology, 121, 63-84.

Soltész, F., Szücs, D., \& Szücs, L. (2010). Relationships between magnitude representation, counting and memory in 4- to 7-year-old children: A developmental study. Behavioral and Brain Functions, 6:13. doi:10.1186/17449081-6-13.

Sowinski C., LeFevre, J.-A., Skwarchuk, S.-L., Kamawar D., Bisanz, J., \& Smith-Chant, B. (2015). Refining the quantitative pathway of the Pathways to Mathematics model. Journal of Experimental Child Psychology, 131, 73-93.

Starkey, P., \& Klein, A. (2008). Sociocultural influences on young children's mathematical knowledge. Contemporary perspectives on mathematics in early childhood education, 253-276.

Tamis-LeMonda, C. S., Sze, I. N. L., Ng, F. F. Y., Kahana-Kalman, R., \& Yoshikawa, H. (2013). Maternal teaching during play with four-year-olds: Variation by ethnicity and family resources. Merrill-Palmer Quarterly, 59(3), 361-398.

Titeca, D., Roeyers, H., Josephy, H., Ceulemans, A., \& Desoete, A. (2014). Preschool predictors of mathematics in first grade children with autism spectrum disorder. Research in Developmental Disabilities, 35(11), 2714-2727. doi:10.1016/j.ridd.2014.07.012 
Tobia, V., Bonifacci, P., \& Marzocchi, G. M. (2016). Concurrent and longitudinal predictors of calculation skills in preschoolers. European Journal of Psychology of Education, 31(2), 155-174.

Toll, S. W., \& Van Luit, J. E. (2012). Early numeracy intervention for low-performing kindergartners. Journal of Early Intervention, 34(4), 243-264.

Vanbinst, K., Ghesquière, P., \& De Smedt, B. (2015). Does numerical processing uniquely predict first graders' future development of single-digit arithmetic?. Learning and Individual Differences, 37, 153-160.

Vanbinst, K., Ansari, D., Ghesquière, P., \& De Smedt, B. (2016). Symbolic numerical magnitude processing is as important to arithmetic as phonological awareness is to reading. PloS one, 11(3), e0151045.

Vanbinst, K., Ceulemans, E., Ghesquière, P., \& De Smedt, B. (2015). Profiles of children's arithmetic fact development: A model-based clustering approach. Journal of experimental child psychology, 133, 29-46.

Vanbinst, K., \& De Smedt, B. (2016). Individual differences in children's mathematics achievement: The roles of symbolic numerical magnitude processing and domaingeneral cognitive functions. Progress in Brain Research.

von Aster, M. G., \& Shalev, R. S. (2007). Number development and developmental dyscalculia. Developmental Medicine and Child Neurology, 49, 868-873.

Wiese, H. (2003). Numbers, language, and the human mind. Cambridge University Press. Wynn, K. (1990). Children's understanding of counting. Cognition, 36, 155-193.

Wynn, K. (1992). Children's acquisition of the number words and the counting system. Cognitive Psychology, 24, 220-251. 
Young-Loveridge, J. M. (2004). Effects on early numeracy of a program using number books and games. Early Childhood Research Quarterly, 19(1), 82-98.

Zhang, X., Koponen, T., Räsänen, P., Aunola, K., Lerkkanen, M.K., \& Nurmi, J. E. (2014).

Linguistic and spatial skills predict early arithmetic development via counting sequence knowledge. Child Development, 85, 1091-1107.

Zhou, X., \& Wang, B. (2004). Preschool children's representation and understanding of written number symbols. Early Child Development and Care, 174, 253-266. doi:10.1080/0300443032000153570. 


\section{APPENDICES}

Appendix A: Frequency distribution for children's age for Study 1.

Appendix B: Stimuli mapping tasks Study 1

Appendix C: Contingency Table for Word-digit and Word-quantity Mapping in Study 1

Appendix D: Frequency distributions of participants' age for Study 2

Appendix E: Stimuli mapping tasks Study 2

Appendix F: Order of presentation for the comparison stimuli Study 2

Appendix G: Sequences of mapping tasks Study 2

Appendix H: Preliminary analyses of direction of mapping Study 2

Appendix I: Correlation table with the six mapping Study 2

Appendix J: Categorical analyses for Word-digit and Word-quantity Mapping in Study 2

Appendix K: Frequency distributions of participants' age for Study 3 


\section{Appendix A}

Frequency distribution of participants' age for Study 1.

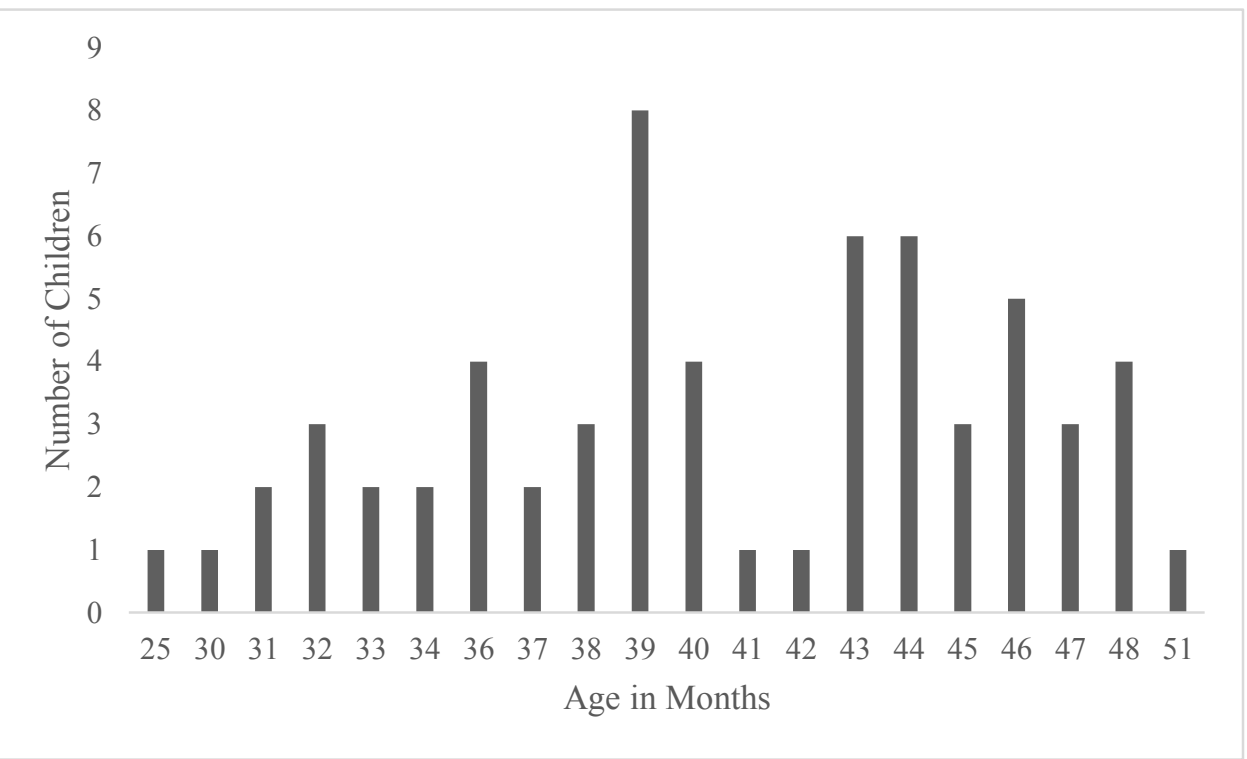




\section{Appendix B}

Stimuli for the mapping tasks in Study 1

\begin{tabular}{|c|c|c|c|}
\hline $\begin{array}{l}\text { Number or } \\
\text { set size }\end{array}$ & Target number or set size & Option 1 & Option 2 \\
\hline \multirow[t]{9}{*}{ Small } & 1 & 1 & 2 \\
\hline & & 3 & 1 \\
\hline & & 1 & 4 \\
\hline & 2 & 2 & 3 \\
\hline & & 4 & 2 \\
\hline & & 2 & 1 \\
\hline & 3 & 4 & 3 \\
\hline & & 3 & 2 \\
\hline & & 1 & 3 \\
\hline \multirow[t]{9}{*}{ Medium } & 4 & 7 & 4 \\
\hline & & 4 & 6 \\
\hline & & 8 & 4 \\
\hline & 5 & 5 & 4 \\
\hline & & 3 & 5 \\
\hline & & 5 & 2 \\
\hline & 6 & 4 & 6 \\
\hline & & 6 & 3 \\
\hline & & 2 & 6 \\
\hline \multirow[t]{9}{*}{ Large } & 7 & 7 & 5 \\
\hline & & 9 & 7 \\
\hline & & 8 & 7 \\
\hline & 8 & 8 & 5 \\
\hline & & 6 & 8 \\
\hline & & 8 & 9 \\
\hline & 9 & 9 & 6 \\
\hline & & 7 & 9 \\
\hline & & 9 & 8 \\
\hline
\end{tabular}




\section{Appendix C}

Contingency Table for Word-digit and Word-quantity Mapping in Study 1

\begin{tabular}{lcc}
\hline & \multicolumn{3}{c}{ Word-quantity } \\
\cline { 2 - 3 } Word-digit & Chance & Above chance \\
\hline Chance & 36 & 0 \\
Above chance & 13 & 13 \\
\hline
\end{tabular}




\section{Appendix D}

Frequency distributions of participants' age: Study 2
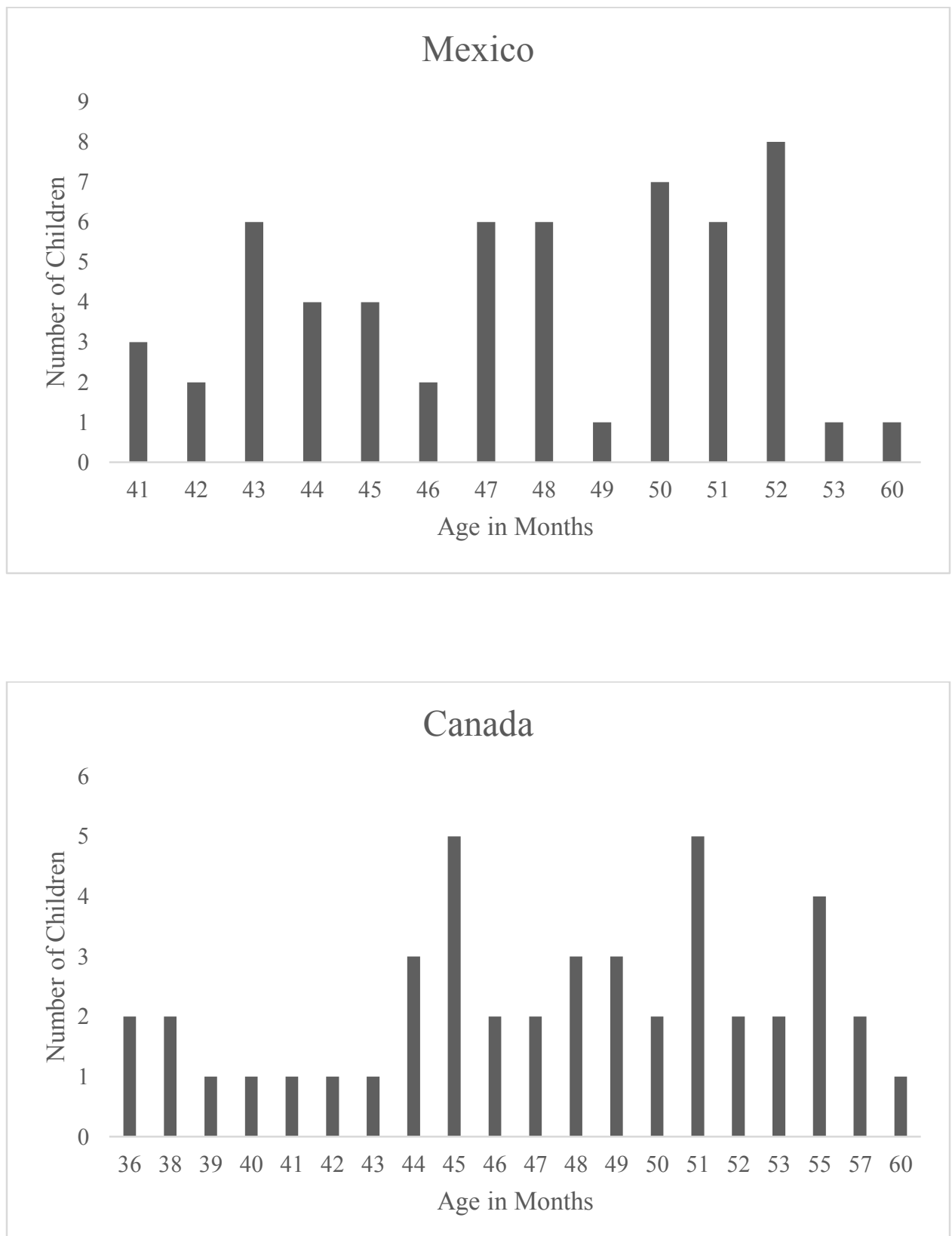


\section{Appendix E}

Stimuli for the mapping tasks in Study 2

\begin{tabular}{|c|c|c|c|}
\hline Number or Set Size & $\begin{array}{l}\text { Target number or set } \\
\text { size }\end{array}$ & Option 1 & Option 2 \\
\hline \multirow[t]{6}{*}{ Small } & 1 & 1 & 3 \\
\hline & 1 & 1 & 2 \\
\hline & 2 & 2 & 3 \\
\hline & 2 & 2 & 4 \\
\hline & 3 & 3 & 4 \\
\hline & 3 & 3 & 2 \\
\hline \multirow[t]{6}{*}{ Medium } & 4 & 4 & 5 \\
\hline & 4 & 4 & 6 \\
\hline & 5 & 5 & 2 \\
\hline & 5 & 5 & 3 \\
\hline & 6 & 6 & 4 \\
\hline & 6 & 6 & 5 \\
\hline \multirow[t]{6}{*}{ Large } & 7 & 7 & 5 \\
\hline & 7 & 7 & 9 \\
\hline & 8 & 8 & 9 \\
\hline & 8 & 8 & 6 \\
\hline & 9 & 9 & 6 \\
\hline & 9 & 9 & 7 \\
\hline
\end{tabular}




\section{Appendix F}

Order of presentation for the comparison stimuli Study 2

\begin{tabular}{cccc}
\hline \multicolumn{4}{c}{ Version A } \\
\hline Distance 1 & Distance 3 & Distance 1 & Distance 3 \\
\hline $2--1$ & $4--1$ & $1--2$ & $1--4$ \\
$4--3$ & $5--2$ & $3--4$ & $2--5$ \\
$6--5$ & $7--4$ & $5--6$ & $4--7$ \\
$8--7$ & $9--6$ & $7--8$ & $6--9$ \\
$9--8$ & $8--5$ & $8--9$ & $5--8$ \\
\hline \multicolumn{5}{c}{} \\
\hline \multicolumn{5}{c}{ Version B } \\
\hline Distance 3 & Distance 3 & Distance 1 \\
\hline $1--4$ & Distance 1 & $4--1$ \\
$4--5$ & $1--2$ & $5--2$ & $4--1$ \\
$6--9$ & $3--4$ & $7--4$ & $6--5$ \\
$5--8$ & $5--6$ & $9--6$ & $8--7$ \\
\hline
\end{tabular}




\section{Appendix G}

Sequences of mapping tasks Study 2

\begin{tabular}{lcccccc}
\hline & \multicolumn{7}{c}{ Sequence } \\
\cline { 2 - 7 } Tasks & $\mathrm{A}$ & $\mathrm{B}$ & $\mathrm{C}$ & $\mathrm{D}$ & $\mathrm{E}$ & $\mathrm{F}$ \\
\hline Word quantity & 1 & 5 & 4 & 1 & 3 & 6 \\
Quantity-word & 2 & 6 & 3 & 2 & 4 & 5 \\
Word-digit & 3 & 2 & 5 & 6 & 1 & 3 \\
Digit-word & 4 & 1 & 6 & 5 & 2 & 4 \\
Digit-quantity & 5 & 4 & 1 & 3 & 6 & 2 \\
Quantity-digit & 6 & 3 & 2 & 4 & 5 & 1 \\
\hline
\end{tabular}




\section{Appendix H}

Preliminary analyses of performance on the mapping tasks Study 2

Table H1. Mapping task means by set size and country

\begin{tabular}{lcccccc}
\hline Task & \multicolumn{5}{c}{ Mexico $(n=57)$} & \multicolumn{3}{c}{ Canada $(n=45)$} \\
\cline { 2 - 7 } & Small & Medium & Large & Small & Medium & Large \\
\cline { 2 - 8 } & $M(S D)$ & $M(S D)$ & $M(S D)$ & $M(S D)$ & $M(S D)$ & $M(S D)$ \\
\hline Digit-Qty & $3.77(1.54)$ & $3.39(1.44)$ & $3.40(1.22)$ & $4.84(1.54)$ & $4.02(1.56)$ & $3.53(1.32)$ \\
Qty-Digit & $3.88(1.51)$ & $3.28(1.41)$ & $2.89(1.25)$ & $5.04(1.24)$ & $4.18(1.42)$ & $3.47(1.50)$ \\
Word-Qty & $4.63(1.40)$ & $3.74(1.25)$ & $3.11(1.38)$ & $5.36(1.03)$ & $4.58(1.39)$ & $3.64(1.43)$ \\
Qty-Word & $5.09(1.21)$ & $3.68(1.68)$ & $3.19(1.37)$ & $5.44(0.94)$ & $4.78(1.38)$ & $3.47(1.59)$ \\
Digit-word & $4.39(1.39)$ & $4.04(1.66)$ & $3.63(1.65)$ & $5.33(1.19)$ & $5.42(0.97)$ & $4.96(1.52)$ \\
Word-Digit & $4.61(1.46)$ & $3.77(1.76)$ & $3.88(1.31)$ & $5.58(1.23)$ & $5.42(1.16)$ & $5.11(1.17)$ \\
\hline
\end{tabular}


Table H2. Statistics for the analyses of each of the mapping pairs.

\begin{tabular}{|c|c|c|c|c|c|c|c|c|c|c|}
\hline & \multicolumn{4}{|c|}{ Quantities-digits } & \multicolumn{3}{|c|}{ Quantities-words } & \multicolumn{3}{|c|}{ Digits-words } \\
\hline & $d f$ & $F$ & $M S E$ & $\eta_{p}^{2}$ & $F$ & $M S E$ & $\eta_{p}^{2}$ & $F$ & $M S E$ & $\eta_{p}^{2}$ \\
\hline Country & 1,100 & $16.84 * * *$ & 4.99 & .14 & $12.31 * *$ & 4.99 & .11 & $36.80 * * *$ & 6.42 & .27 \\
\hline Direction & 1,100 & 0.17 & 1.19 & .00 & 1.31 & 1.16 & .01 & 1.31 & 1.19 & .01 \\
\hline C x Dir & 1,100 & 2.24 & 1.19 & .02 & 0.52 & 1.16 & .01 & 0.13 & 1.19 & .00 \\
\hline $\operatorname{Size}^{\mathrm{a}}$ & 2,200 & $36.44 * * *$ & 1.59 & .27 & $117.89 * * *$ & 1.35 & .54 & $14.69 * * *$ & 1.26 & .13 \\
\hline $\mathrm{SxC}$ & 2,200 & $4.70 *$ & 1.59 & .05 & $3.20 *$ & 1.35 & .03 & $3.43 *$ & 1.26 & .03 \\
\hline DxS & 2,200 & 1.93 & 1.34 & .02 & 1.18 & 1.10 & .01 & 2.06 & 1.00 & .02 \\
\hline $\mathrm{CxDxS}$ & 2,200 & 0.28 & 1.34 & .00 & 1.26 & 1.10 & .01 & 0.41 & 1.00 & .00 \\
\hline
\end{tabular}




\section{Appendix I}

Zero order correlations among numeracy measures Study 2.

\begin{tabular}{|c|c|c|c|c|c|c|c|c|c|c|}
\hline & Count & Digit-Qty & Qty-Digit & Word-Qty & Qty-Word & Digit-Word & Word-digit & Give $\mathrm{N}$ & KeyMath & V. Comp \\
\hline Count & -- & $.54 * * *$ & $.58 * * *$ & $.40^{* *}$ & $.57 * * *$ & $.43^{* *}$ & $.52 * * *$ & $.69 * * *$ & $.72 * * *$ & $.44 * *$ \\
\hline Digit-Qty & $.44^{* *}$ & -- & $.59 * * *$ & $.35^{* *}$ & $.62 * * *$ & $.57 * * *$ & $.64 * * *$ & $.55 * * *$ & $.66^{* * *}$ & $.31 *$ \\
\hline Qty-Digit & .23 & $.64 * * *$ & -- & $.34 *$ & $.48 * * *$ & $.46^{* * *}$ & $.50 * * *$ & $.49 * * *$ & $.59 * * *$ & $.45 * * *$ \\
\hline Word-Qty & $.42 * *$ & $.63^{* * *}$ & $.65^{* * *}$ & -- & $.68^{* * *}$ & $.50^{* * *}$ & $.48^{* * *}$ & $.46^{* * *}$ & $.52 * * *$ & $.54 * * *$ \\
\hline Qty-Word & $.43 * *$ & $.65^{* * *}$ & $.59 * * *$ & $.53 * * *$ & -- & $.64 * * *$ & $.61 * * *$ & $.65 * * *$ & $.71 * * *$ & $.63 * * *$ \\
\hline Digit-Word & $.34 *$ & $.63 * * *$ & $.51 * * *$ & $.50 * * *$ & $.49 * *$ & -- & $.70 * * *$ & $.48^{* * *}$ & $.60 * * *$ & $.53 * * *$ \\
\hline Word-Digit & $.47 * * *$ & $.61 * * *$ & $.45^{* *}$ & $.58 * * *$ & $.59 * * *$ & $.66^{* * *}$ & -- & $.55^{* * *}$ & $.69 * * *$ & $.50 * * *$ \\
\hline Give $\mathrm{N}$ & $.45^{* *}$ & $.50 * * *$ & $.54 * * *$ & $.68 * * *$ & $.53 * * *$ & $.46^{* *}$ & $.62 * * *$ & -- & $.74 * * *$ & $.50 * * *$ \\
\hline KeyMath & $.57 * * *$ & $.62 * * *$ & $.57 * * *$ & $.65 * * *$ & $.68 * * *$ & $.54 * * *$ & $.53 * * *$ & $.71 * * *$ & -- & $.60 * * *$ \\
\hline V. Comp & $.55 * * *$ & $.70 * * *$ & $.62 * * *$ & $.67 * * *$ & $.69 * * *$ & $.53 * * *$ & $.56^{* * *}$ & $.63 * * *$ & $.67 * * *$ & -- \\
\hline
\end{tabular}

$* p<.05, * * p<.01, * * * p<.001$

Note: Mexican sample above the diagonal; Canadian sample below the diagonal. 


\section{Appendix J}

Categorical analyses for word-digit, word-quantity mapping in Study 2

Logistic regression analyses showed that for the Mexican children, performance on the word-digit mapping significantly predicted performance on the word-quantity mapping task $B=2.23, S E=0.75$, Wald $=8.78, p=.003$, Odds Ratio $=9.27$ and $95 \% \mathrm{CI}$ [2.13-40.45]. The probability of successfully mapping words to quantities was $8 \%$ if children were not successful at mapping words to digits. In contrast, children who successfully mapped words-digits had a $45 \%$ probability of mapping words-quantities.

Logistic regression analyses predicting word quantity mapping from word-digit mapping for the Canadian children were not performed due to the observed complete separation (See Table K1).

Table K1. Contingency table for word-digit, word-quantity mapping by country.

$$
\text { Word-quantity }
$$

Mexico Canada

\begin{tabular}{lcccc}
\hline Word-digit & Chance & Above chance & Chance & Above chance \\
Chance & 34 & 3 & 9 & 0 \\
Above chance & 11 & 9 & 16 & 20
\end{tabular}




\section{Appendix K}

Frequency Distribution of Participants' Age for Study 3

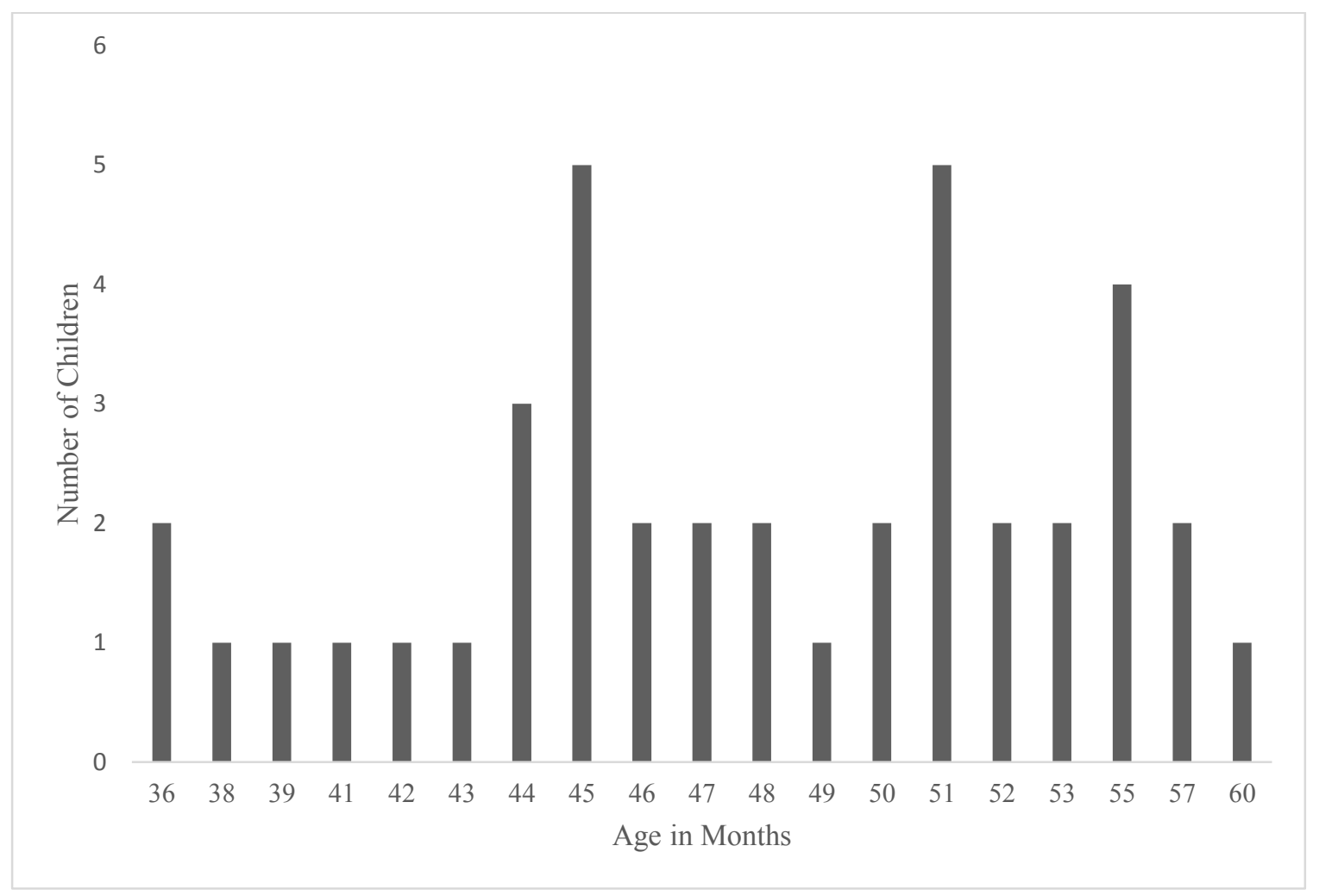

